\title{
Laser-assisted spin-polarized transport in graphene tunnel junctions
}

\author{
Kai-He Ding ${ }^{1,2}$, Zhen-Gang Zhu ${ }^{1}$, and Jamal Berakdar ${ }^{1}$ \\ ${ }^{1}$ Institut für Physik, Martin-Luther-Universität Halle-Wittenberg, 06099 Halle \\ (Saale), Germany \\ ${ }^{2}$ Department of Physics and Electronic Science, Changsha University of Science and \\ Technology, Changsha,410076, China
}

\begin{abstract}
The Keldysh nonequilibrium Green's function method is utilized to study theoretically the spin polarized transport through a graphene spin valve irradiated by a monochromatic laser field. It is found that the bias dependence of the differential conductance exhibits two peaks corresponding to the resonant tunneling through the photon-assisted subbands. A zero value plateau in the differential conductance appears symmetrically on the zero bias due to the dynamical gap opened by the radiation field, and its width can be tuned by changing the radiation intensity and frequency, which leads to the shift of the resonant peaks in the differential conductance as well. We demonstrate that the shift of the resonant peaks in the differential conductance with the radiation intensity exhibits different features from that with the radiation frequency. We also show numerically the dependencies of the radiation and spin valve effects on the parameters of the external fields and those of the electrodes. We find that the combined effects of the radiation field, the graphene, and the spin valve properties bring about a plateau in the tunnel magnetoresistance, and the width of this plateau can be changed by scanning the radiation field strength and/or the frequency.

PACS numbers: 85.75.-d, 81.05.Uw, 75.47.-m
\end{abstract}




\section{Introduction}

Electromagnetic radiation was demonstrated to be a powerful tool for the exploration and modification of materials properties. For example, in conventional semiconducting nanostructures a variety of applications have been established such as the various forms of photo electronic devices (e.g., the radiation-controlled field-effect transistors, photodiodes, and the light-emitting diodes [1, 2, 3, 4, 5]). On the other hand, graphene with its many unusual physical properties (to mention but a few, the half integer quantum Hall effect [6], the Klein tunneling [7] and the conductance properties [8]) offers a new platform to explore radiation effects. The influence of electromagnetic fields on graphene has been recently a subject of intense research. Particularly interesting examples are the linear response to and the frequency dependence of the conductivity [9, 10, 11], the photon-assisted transport [12, 13], the microwave and far-infrared response [14, 15, 16], the plasmon spectrum [17, 18, 19], as well as the nonlinear response to the electromagnetic radiation [20, 21, 22, 23]. The potential applications of graphene in terahertz electronics were pointed out in Refs. 24].

The resonant interaction between graphene and an electromagnetic field may open a dynamical gap in the quasiparticle spectrum of graphene [25], which leads to a strong suppression of the quasiparticle transmission through a graphene $\mathrm{p}-\mathrm{n}$ junction. However, a directed current without applying any dc bias voltage can be generated in certain conditions as a result of inelastic quasiparticle tunneling assisted by one- or two-photon absorptions [26]. Another way to generate a direct current is to use shaped, timely tuned electromagnetic pulses [23] which also may generate a valley current. Therefore, it seems possible to control the transport properties of diverse graphene tunneling structures by the variation of the strength and the frequency of the external radiation fields. On

the other hand, the potential of graphene for spin-dependent transport (spintronic) applications is well documented by now [27, 28, 30, 29, 31, 32, 33, 34, 35]. Motivated by these facts, in this work, we study theoretically the spin polarized transport through a graphene-spin-valve device in the presence of a monochromatic laser field. The method is based on the standard Keldysh nonequilibrium Green's function approach, as described in Refs. [37] and [38]. It is found that the bias dependence of the differential conductance exhibits two implicit peaks corresponding the resonant tunneling through the photoninduced subbands. The resonant interaction of the quasiparticle in graphene with the radiation field turns on a dynamical gap in the quasiparticle spectrum. When the bias voltage lies inside this gap region, the differential conductance displays a zero value plateau situated symmetrically on the zero bias. The width of this plateau can be tuned by changing radiation intensity and frequency, which also causes a shift of the resonant peaks in the differential conductance. The tunnel magnetoresistance (TMR) versus the bias voltage exhibits a plateau around the zero bias voltage, and its width can be controlled by the radiation field strength and/or the frequency. 


\section{Theoretical model}

We consider a spin valve device consisting of an extended graphene sheet (that defines the $x-y$ plane) contacted by two ferromagnetic electrodes. A gate voltage $V_{g}$ applied to graphene shifts the Dirac point away from the zero energy. The two electrodes are voltage-biased with respect to each other with a bias $V$. The electrical current flows in the $x$ direction. Additionally, we assume that a laser field is irradiating homogenously the structure. Within the metallic electrodes we assume that the field is shielded and ignore thus its effect on the electrodes. The laser field is monochromatic and is linearly polarized along the $y$ direction. The low energy graphene Hamiltonian around the Dirac points has the minimal coupling form

$$
H_{G}=v_{F} \boldsymbol{\sigma} \cdot \mathbf{k}-e v_{F} A(t) \sigma_{y}
$$

where $\mathbf{k}$ is the operator of the electron momentum in the graphene plane, $e$ is the electron charge, $\boldsymbol{\sigma}$ is the vector build out of the Pauli matrices in the sublattice space, and $A$ is the laser's vector potential. In the tight binding description [39], $v_{F}=\frac{3}{2} a t_{g}$ with $t_{g}$ being the nearest neighbor hopping energy, and $a$ being the carbon-carbon distance. The vector potential $A(t)$ is taken as

$$
A(t)=\frac{E_{0}}{\omega_{0}} \sin \omega_{0} t
$$

where $\omega_{0}$ is the frequency of the radiation field, and $E_{0}$ is its amplitude. Diagonalizing the Hamiltonian (1) in the absence of the radiation field, one finds the eigenvalues

$$
\epsilon_{s \mathbf{k}}=s v_{F}|\mathbf{k}|+V_{g}
$$

with $s= \pm$ denoting the band index, and the eigenstates

$$
\psi_{s \mathbf{k}}(\mathbf{r})=\frac{1}{\sqrt{\Omega}} e^{i \mathbf{k} \cdot \mathbf{r}} u_{\mathbf{k}}^{s}
$$

where $\Omega$ is the volume of the system, and

$$
u_{\mathbf{k}}^{s}=\frac{\sqrt{2}}{2}\left(\begin{array}{c}
1 \\
s e^{i \phi(\mathbf{k})}
\end{array}\right), \quad \tan \phi(\mathbf{k})=\frac{k_{y}}{k_{x}} .
$$

We introduce the field operators

$$
\Psi_{\tau}(\mathbf{r}, t)=\sum_{\mathbf{k}, s} a_{s \mathbf{k} \tau}(t) \psi_{s \mathbf{k}}(\mathbf{r}),
$$

where $a_{s \mathbf{k} \tau}$ is the usual annihilation operator for an electron in the band $s$, with the momentum $\mathbf{k}$ and the spin $\tau$, and then express the Hamiltonian (1) in the second quantized form as

$$
H_{G}=\sum_{\mathbf{k} s \tau} \epsilon_{s \mathbf{k}} a_{s \mathbf{k} \tau}^{\dagger} a_{s \mathbf{k} \tau}-e v_{F} A(t) \sum_{\mathbf{k} s s^{\prime} \tau} d_{s s^{\prime}, \mathbf{k}} a_{s \mathbf{k} \tau}^{\dagger} a_{s^{\prime} \mathbf{k} \tau}
$$

where

$$
d_{s s^{\prime}, \mathbf{k}}=\frac{i}{2}\left(s e^{-i \phi(\mathbf{k})}-s^{\prime} e^{i \phi(\mathbf{k})}\right)
$$


Applying the rotating wave approximation [40], we neglect the energy nonconserving terms. Accounting for the coupling between the graphene and the two ferromagnetic electrodes we write for the Hamiltonian of the complete tunnel junction in the presence of the laser

$$
H=H_{G}+H_{L}+H_{R}+H_{T}
$$

where

$$
\begin{aligned}
H_{G}= & \sum_{\mathbf{k} s \tau} \epsilon_{s \mathbf{k}} a_{s \mathbf{k} \tau}^{\dagger} a_{s \mathbf{k} \tau} \\
& -e v_{F} \sum_{\mathbf{k} \tau}\left\{A_{0}^{*} e^{-i \omega_{0} t} d_{+-, \mathbf{k}} a_{+\mathbf{k} \tau}^{\dagger} a_{-\mathbf{k} \tau}\right. \\
& \left.+A_{0} e^{i \omega_{0} t} d_{-+, \mathbf{k}} a_{-\mathbf{k} \tau}^{\dagger} a_{+\mathbf{k} \tau}\right\}
\end{aligned}
$$

with $A_{0}=-i \frac{E_{0}}{2 \omega_{0}}$, and

$$
\begin{aligned}
& H_{\lambda}=\sum_{\mathbf{q} \tau} \varepsilon_{\mathbf{q} \lambda \tau} c_{\mathbf{q} \lambda \tau}^{\dagger} c_{\mathbf{q} \lambda \tau}, \quad \lambda=L, R, \\
& H_{T}=\frac{1}{\sqrt{N}} \sum_{\mathbf{q} \mathbf{k} \lambda \tau}\left(T_{\mathbf{k} \lambda \mathbf{q}} c_{\mathbf{q} \lambda \tau}^{\dagger} a_{s \mathbf{k} \tau}+\text { H.c. }\right) .
\end{aligned}
$$

Equations (11) and (12) describe respectively the $\lambda$ electrode and the coupling between the graphene and the electrodes. $\varepsilon_{\mathbf{q} \lambda \tau}$ is the single electron energy, $c_{\mathbf{q} \lambda \tau}^{\dagger}\left(c_{\mathbf{q} \lambda \tau}\right)$ is the usual creation (annihilation) operator for an electron with the momentum $\mathbf{q}$ and the spin $\tau$ in the $\lambda$ electrode; $N$ is the number of sites on the sublattice.

By introducing a unitary transformation

$$
U=\exp \left[-i \frac{\omega_{0} t}{2} \sum_{\mathbf{k} \tau}\left(a_{+\mathbf{k} \tau}^{\dagger} a_{+\mathbf{k} \tau}-a_{-\mathbf{k} \tau}^{\dagger} a_{-\mathbf{k} \tau}\right)\right],
$$

we redefine the Hamiltonian of the system in the rotating reference as

$$
\begin{aligned}
\widetilde{H} & =U^{-1} H U+i \frac{d U^{-1}}{d t} U \\
& =\sum_{\mathbf{k} \tau} \widetilde{\epsilon}_{s \mathbf{k}} a_{s \mathbf{k} \tau}^{\dagger} a_{s \mathbf{k} \tau}+\sum_{\mathbf{k} \tau} \Delta\left(a_{+\mathbf{k} \tau}^{\dagger} a_{-\mathbf{k} \tau}+a_{-\mathbf{k} \tau}^{\dagger} a_{+\mathbf{k} \tau}\right) \\
& +\sum_{\mathbf{q} \lambda \tau} \varepsilon_{\mathbf{q} \lambda \tau} c_{\mathbf{q} \lambda \tau}^{\dagger} c_{\mathbf{q} \lambda \tau}+\frac{1}{\sqrt{N}} \sum_{\mathbf{k} \mathbf{q} \lambda s \tau}\left(T_{\mathbf{k} \lambda s \mathbf{q}}(t) c_{\mathbf{q} \lambda \tau}^{\dagger} a_{s \mathbf{k} \tau}+H . c .\right)
\end{aligned}
$$

where

$$
\Delta=\frac{e v_{F} E_{0}}{2 \omega_{0}}, T_{\mathbf{k} \lambda s \mathbf{q}}(t)=T_{\mathbf{k} \lambda \mathbf{q}} e^{\frac{-i s \omega_{0} t}{2}}
$$

and

$$
\widetilde{\epsilon}_{s \mathbf{k}}=\epsilon_{s \mathbf{k}}-s \omega_{0} / 2
$$

In the calculation of equation (14), we have assumed (as in Refs. [25, 26, 36]) that the most important contributions stem from an almost one dimensional electron motion $\left(k_{x} \gg k_{y}\right)$ in the interaction with the radiation field. 
The electric current of the system can be calculated from the time evolution of the occupation number operator of the left electrode,

$$
I_{L}=e\left\langle\dot{\mathcal{N}}_{L}\right\rangle=\frac{i e}{\hbar}\left\langle\left[\widetilde{H}, \mathcal{N}_{L}\right]\right\rangle \text {, where } \mathcal{N}_{L}=\sum_{\mathbf{q} \tau} c_{\mathbf{q} L \tau}^{\dagger} c_{\mathbf{q} L \tau} .
$$

Using the nonequilibrium Green's function method, equation (15) can be further expressed as

$$
\begin{aligned}
I_{L}= & -\frac{i e}{\hbar} \sum_{s s^{\prime}} \int d t_{1} \int \frac{d \varepsilon}{2 \pi} \operatorname{Tr}\left\{\left[\left(\mathcal{G}_{\mathrm{s}, \mathrm{s}^{\prime}}^{\mathrm{r}}\left(\mathrm{t}, \mathrm{t}_{1}\right)-\mathcal{G}_{\mathrm{s}, \mathrm{s}^{\prime}}^{\mathrm{a}}\left(\mathrm{t}, \mathrm{t}_{1}\right)\right) \mathrm{f}_{\mathrm{L}}(\varepsilon)\right.\right. \\
& \left.\left.+\mathcal{G}_{s, s^{\prime}}^{<}\left(t, t_{1}\right)\right] \Gamma_{L}\right\} e^{-i \varepsilon\left(t_{1}-t\right)} e^{-\frac{i s \omega_{0} t}{2}} e^{\frac{i s^{\prime} \omega_{0} t_{1}}{2}}
\end{aligned}
$$

where $\operatorname{Tr}$ is the trace in the spin space, $f_{\lambda}(\varepsilon)$ is the Fermi distribution, $\mathcal{G}_{s, s^{\prime}}^{r(a)}\left(t, t_{1}\right)=$ $\frac{1}{N} \sum_{\mathbf{k k}^{\prime}} G_{\mathbf{k} s, \mathbf{k}^{\prime} s^{\prime}}^{r(a)}\left(t, t_{1}\right)$, and $\mathcal{G}_{s, s^{\prime}}^{<}\left(t, t_{1}\right)=\frac{1}{N} \sum_{\mathbf{k k}^{\prime}} G_{\mathbf{k} s, \mathbf{k}^{\prime} s^{\prime}}^{<}\left(t, t_{1}\right)$ are $2 \times 2$ matrices denoting the retarded (advanced) Green's function and the lesser Green's function, respectively. In the calculation of equation (16), we assume that the dominant contributions to the tunneling stem from the electrons near the Fermi level, and hence the linewidth function to be independent of $\mathbf{k}$. Thus, we have

$$
\Gamma_{\lambda}=\left(\begin{array}{cc}
\Gamma_{\lambda}^{\uparrow} & 0 \\
0 & \Gamma_{\lambda}^{\downarrow}
\end{array}\right)
$$

with $\Gamma_{\lambda}^{\tau}=2 \pi \sum_{\mathbf{q}} T_{\mathbf{k} \lambda \mathbf{q}}^{*} T_{\mathbf{k}^{\prime} \lambda \mathbf{q}} \delta\left(\varepsilon-\varepsilon_{\mathbf{q} \lambda \tau}\right)$. In order to solve equation (16)), we need to calculate the Green's functions $\mathcal{G}_{s s^{\prime}}^{\tau \tau^{\prime}, r}\left(t, t^{\prime}\right)$ and $\mathcal{G}_{s s^{\prime}}^{\tau \tau^{\prime},<}\left(t, t^{\prime}\right)$. Using the equation of motion method, we get

$$
\begin{aligned}
& \mathcal{G}^{\tau \tau^{\prime}, r}\left(t, t^{\prime}\right)=\delta_{\tau \tau^{\prime}} g^{\tau \tau^{\prime}, r}\left(t-t^{\prime}\right) \\
& +\int d t_{1} d t_{2} g^{\tau \tau, r}\left(t-t_{1}\right) \Sigma^{\tau, r}\left(t_{1}, t_{2}\right) \mathcal{G}^{\tau \tau^{\prime}, r}\left(t_{2}, t^{\prime}\right)
\end{aligned}
$$

where

$$
\Sigma_{s s^{\prime}}^{\tau, r}\left(t, t^{\prime}\right)=\int \frac{d \varepsilon}{2 \pi} \Sigma_{0}^{\tau, r} e^{-i \varepsilon\left(t-t^{\prime}\right)} e^{i \frac{s \omega_{0} t}{2}} e^{-i \frac{s^{\prime} \omega_{0} t^{\prime}}{2}}
$$

with $\Sigma_{0}^{\tau, r}=-\frac{i}{2}\left(\Gamma_{L}^{\tau}+\Gamma_{R}^{\tau}\right)$, and $g^{\tau \tau^{\prime}, r}(t)$ is the retarded Green's function of graphene without the coupling of the electrodes, and can be obtained by a straightforward calculation. The detailed expressions are given in the Appendix. $\mathcal{G}^{\tau \tau^{\prime},<}\left(t, t^{\prime}\right)$ is related to $\mathcal{G}^{\tau \tau^{\prime}, r}\left(t, t^{\prime}\right)$ through the Keldysh equation

$$
\mathcal{G}^{\tau \tau,<}\left(t, t^{\prime}\right)=\int d t_{1} d t_{2} G^{\tau \tau, r}\left(t, t_{1}\right) \Sigma^{\tau,<}\left(t_{1}, t_{2}\right) G^{\tau \tau, a}\left(t_{2}, t^{\prime}\right)
$$

where

$$
\begin{aligned}
\Sigma_{s s^{\prime}}^{\tau,<}\left(t, t^{\prime}\right)= & i \int \frac{d \varepsilon}{2 \pi}\left[\Gamma_{L}^{\tau} f_{L}(\varepsilon)+\Gamma_{R}^{\tau} f_{R}(\varepsilon)\right] \\
& \times e^{-i \varepsilon\left(t-t^{\prime}\right)} e^{i \frac{s \omega_{0} t}{2}} e^{-i \frac{s^{\prime} \omega_{0} t^{\prime}}{2}} .
\end{aligned}
$$

Notice that the Green's functions in equations (18) and (20) do not depend only on the difference of the two time variables, thus one should take a generalized Fourier expansion as 41

$$
\mathcal{G}\left(t, t^{\prime}\right)=\frac{1}{2 \pi} \sum_{n} \int d \varepsilon e^{-i \varepsilon t} e^{i\left(\varepsilon+n \omega_{0} / 2\right) t^{\prime}} \mathcal{G}\left(\varepsilon, \varepsilon+n \omega_{0} / 2\right) .
$$


Hereafter we shall use the simple notation

$$
\mathcal{G}_{n m}(\varepsilon)=\mathcal{G}\left(\varepsilon+n \omega_{0} / 2, \varepsilon+m \omega_{0} / 2\right)
$$

Evidently, the different Fourier components satisfy the relation

$$
\mathcal{G}_{n m}(\varepsilon)=\mathcal{G}_{n-m, 0}\left(\varepsilon+m \omega_{0} / 2\right) .
$$

From equation (18), the Fourier components of the Green's function can be expressed as

$$
\begin{aligned}
\mathcal{G}_{m, n}^{\tau \tau, r}= & g_{m n}^{\tau \tau, r} \\
& +\widetilde{\epsilon}_{m} \mathcal{G}_{m, n}^{\tau \tau, r}+V_{m, m-2} \mathcal{G}_{m-2, n}^{\tau \tau, r}+V_{m, m+2} \mathcal{G}_{m+2, n}^{\tau \tau, r},
\end{aligned}
$$

where $\widetilde{\epsilon}_{m}=g_{m n}^{\tau \tau, r} \Sigma_{0}^{\tau, r}$, and

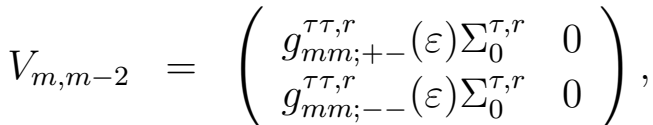

$$
\begin{aligned}
& V_{m, m+2}=\left(\begin{array}{cc}
0 & g_{m m ;++}^{\tau \tau, r}(\varepsilon) \Sigma_{0}^{\tau, r} \\
0 & g_{m m ;-+}^{\tau \tau, r}(\varepsilon) \Sigma_{0}^{\tau, r}
\end{array}\right),
\end{aligned}
$$

with $g_{m n, s s^{\prime}}^{\tau \tau, r}(\varepsilon)=\delta_{m n} g_{s s^{\prime}}^{\tau \tau, r}\left(\varepsilon+m \omega_{0} / 2\right)$. Note that equation (23) is formally equivalent to the ones describing the motion of electrons in a tight-binding linear chain with site energies and the nearest-neighbor coupling. Its solution can be derived by using the conventional recursive technique [41.

Let $\Gamma_{L}^{\tau}=\gamma \Gamma_{R}^{\tau}$, and $N(t)$ be the occupation of the central graphene. By using the continuity equation,

$$
\frac{d N(t)}{d t}=I_{R}(t)+I_{L}(t)
$$

we finally obtain the time-averaged current

$$
\langle I\rangle=-\frac{\gamma}{1+\gamma} \frac{i e}{\hbar} \sum_{s s^{\prime} \tau} \int \frac{d \varepsilon}{2 \pi}\left[\mathcal{G}_{-s,-s^{\prime} ; s s^{\prime}}^{\tau \tau, r}(\varepsilon)-\mathcal{G}_{-s,-s^{\prime} ; s s^{\prime}}^{\tau \tau, a}(\varepsilon)\right] \Gamma_{R}^{\tau}\left[f_{L}(\varepsilon)-f_{R}(\varepsilon)\right],
$$

where $\mathcal{G}_{m n ; s s^{\prime}}^{\tau \tau, r}(\varepsilon)$ is the matrix elements of $\mathcal{G}_{m n}^{\tau \tau, r}(\varepsilon)$. In equation (24), we further assume a symmetrical voltage division: $\mu_{L ; R}=E_{F} \pm 1 / 2 \mathrm{eV}$, and put $E_{F}=0$ in the numerical calculations. The expression (24) is the central result of this paper, and allows one to describe the spin-polarized transport within the rotating wave approximation. WE note the difference to our previous study [13] in which we studied the influence of a time-dependent chemical potential (realized as an oscillating bias voltage). In the present study the radiation field, coupled to the carrier as a vector potential, induces a dynamical gap in the quasiparticle spectrum. This, in turn, leads to changes in the transport properties and TMR, as shown below. The TMR is deduced according to the conventional definition

$$
\mathrm{TMR}=\frac{\mathrm{I}(0)-\mathrm{I}(\pi)}{\mathrm{I}(0)},
$$

where $I(0(\pi))$ is the time-averaged current flowing through the system in the parallel (antiparallel) configuration. 


\section{Numerical analysis}

Based on the above analytical expressions, we present the numerical calculations for the spin polarized transport through the system. Firstly, we assume the linewidth function $\Gamma_{\lambda}^{\tau}(\varepsilon)$ to be independent of the energy within the wide band approximation, and the two electrodes are made of the same material. Introducing the degree of the spin polarizations of the left and the right electrodes $p_{L}=p_{R}=p$, we can write

$\Gamma_{L}^{\uparrow \downarrow}=\Gamma_{R}^{\uparrow \downarrow}=\Gamma_{0}(1 \pm p)$ where $\Gamma_{0}$ describes the coupling between the graphene and the electrodes in the absence of an internal magnetization. We set the temperature to zero and $t_{g} \approx 2.8 \mathrm{eV}$ in our calculation. In addition, we choose the frequency $\hbar \omega_{0} \leq 0.06 t_{g}$, and the voltage $e V<1.4 t_{g}$ so that the Dirac-type behaviour is maintained in this energy regime 39.

Fig:1(a) shows the the differential conductance $G=d\langle I\rangle / d V$ as a function of the bias voltage for different radiation strengths in the parallel configuration of the two electrodes. In the absence of the radiation field, the differential conductance exhibits a linear dependence on the bias voltage (see Fig:1(a)). The graphene spin valve device is sensitive to the electromagnetic radiation. When the radiation field is applied, some characteristic features are presented explicitly in the transport. Firstly, close to the zero bias voltage, a salient zero value region appears symmetrically in the differential conductance, and the width of the region becomes larger with increasing the radiation strength. This is because the resonant interaction between the graphene and the external radiation field opens a dynamical gap in the spectrum of the quasiparticle in the graphene. When the bias voltage lies inside this gap, the graphene can be viewed as an insulator, thus the electron transport through the graphene is suppressed. Secondly, the differential conductance as a function of the bias voltage exhibits two peaks corresponding to the resonant tunneling via the photon-induced sidebands. This behavior is different to the case of the presence of an oscillating gate voltage in graphene sheet (a successive peaks are observed ) 13. The reason for this difference is that the sidebands in the latter case are produced due to the modulation of each quasiparticle level by the gate voltage; while in former case they are caused by the electron scattering between the sidebands. When the electrons are injected from the electrode to the graphene, the radiation field results in multiple electron scattering events and two resonant points within our approximation [26]; for higher intensities that may result in further structures one has to go beyond the rotating wave approximation. When the bias windows cross the resonant points, the resonant tunnelings happen leading to the appearance of two peaks. In addition, for the case of the gate voltage, when changing the amplitude of the gate voltage, the positions of the peaks are fixed. In the present case, with increasing the radiation strength, the positions of the peaks shift in the direction away from the origin. This is due to the increase of the dynamical gap lifting the photon-assisted subbands. Fig.1(b) shows the bias dependence of the differential conductance for the different frequency $\omega_{0}$ in the parallel configuration of the electrodes. With increasing the frequency, the dynamical gap diminishes leading to the 


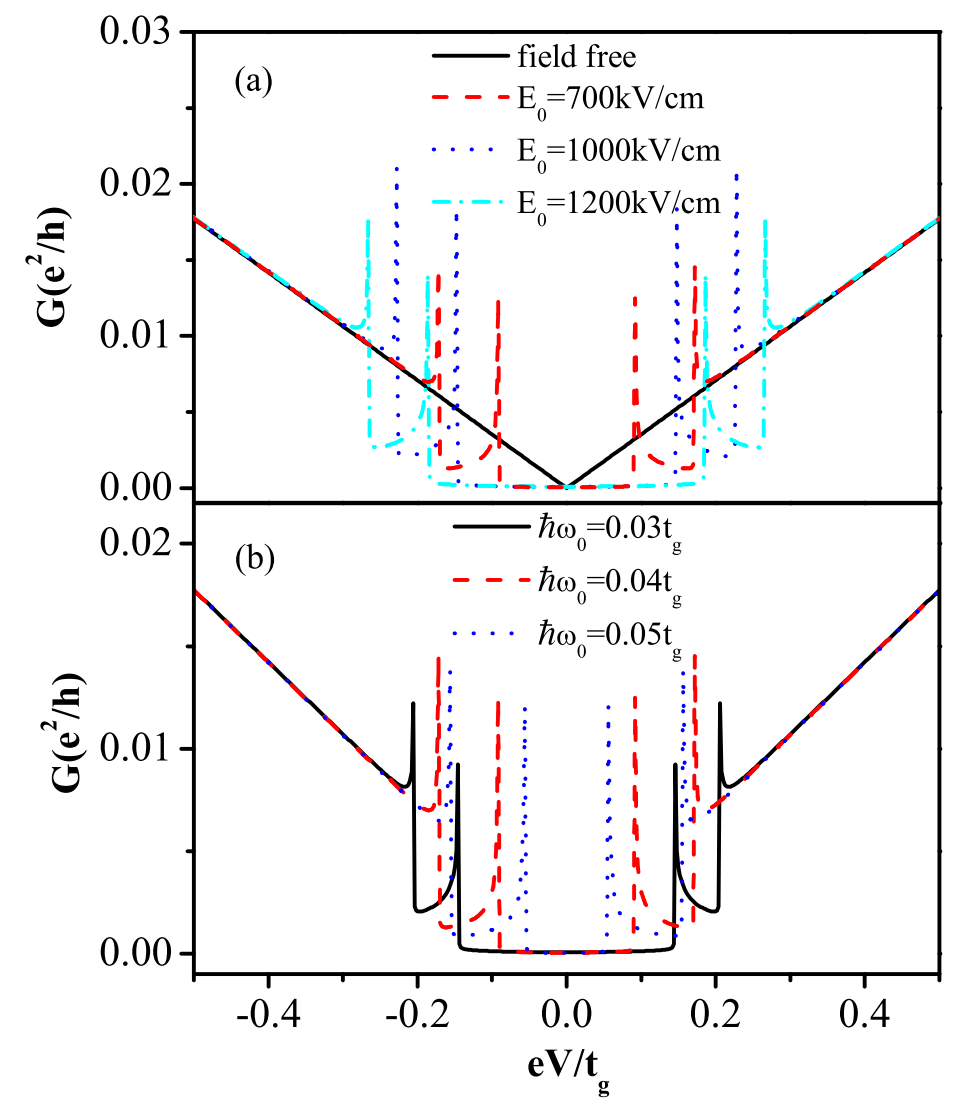

Figure 1. (color online)The bias dependence of the differential conductance $G$ for different radiation strength $E_{0}$ at $\hbar \omega_{0}=0.04 t_{g}(\mathrm{a})$, and for different radiation frequency $\omega_{0}$ at $E_{0}=700 \mathrm{kV} / \mathrm{cm}$ (b). The other parameters are taken as $P=0.4, \Gamma_{0}=0.05 t_{g}$, $V_{g}=0$ and $D=3 t_{g}$. The inset in (a) shows the differential conductance in the absence of the radiation field.

decrease of the width of zero value plateau and the shift of the peaks towards the origin. Especially, one can find that the amplitude of the peaks change non-monotonously with the radiation intensity and the frequency. The reason is that the decrease of the frequency or the increase of the radiation intensity enhances the gap, which lifts the photon-induced subbands, and causes the increase of the density of state for the subbands, thus enhancing the electrons tunneling through the graphene.

Fig.2(a) shows the differential conductance as a function of the bias voltage for the different coupling strength $\Gamma_{0}$ between the graphene and the electrodes in the parallel configuration of the electrodes. With increasing the coupling strength, the electrons tunnel more easily from the electrode to the graphene, thus leading to the rise of the differential conductance with $\Gamma_{0}$. While the zero value plateau in the differential conductance remains the same because the coupling between the electrode and the graphene has no influence on the dynamical gap in the quasiparticle spectrum of the graphene. Figure 2(b) illustrates the bias dependence of the differential conductance for different gate voltages. It shows that the oscillation peaks and the zero value region shift towards the positive direction of the bias voltage. 

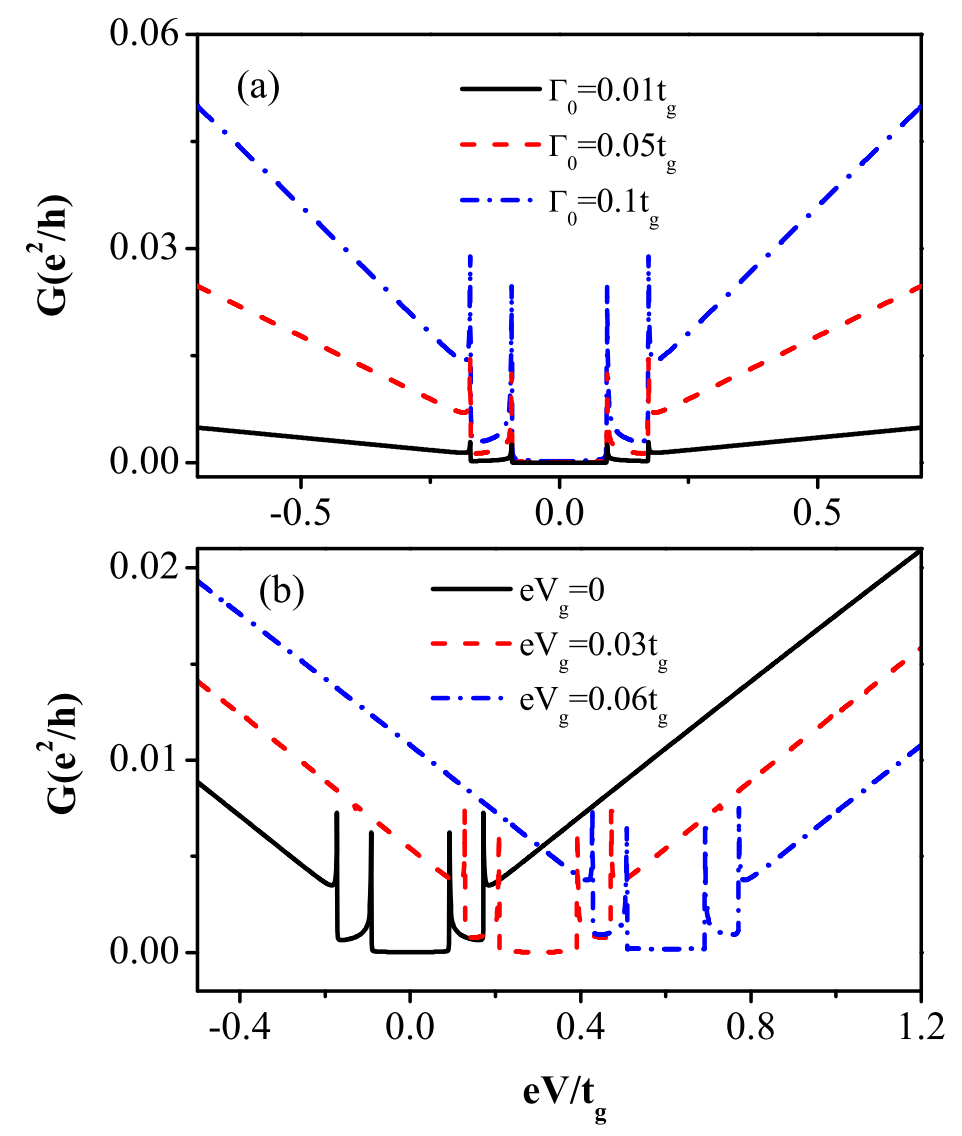

Figure 2. (color online) The differential conductance $G$ versus the bias voltage (a) for coupling strength $\Gamma_{0}$ at $V_{g}=0$, and (b) for different gate voltages $V_{g}$ at $\Gamma_{0}=0.05 t_{g}$. The other parameters are taken the same as those of Fig 1.

The differential conductance as a function of the radiation intensity for the different radiation frequency in the parallel configuration of the electrodes is shown in Fig. 3(a). Two resonant peaks via the photon-induced sidebands can be distinctly observed. The dynamical gap in the quasiparticle spectrum of the graphene becomes larger with increasing the radiation strength, up-shifting the subbands induced by the radiation field. When the subbands fall into the bias windows, resonant tunneling occurs, manifested as the conductance peaks. For radiation with higher frequencies, the resonant peaks appear at larger $E_{0}$ in accordance with a larger dynamical gap. When $E_{0}$ is large enough, the differential conductance tends to a constant value. Additionally, it is found that the interval between the resonant peaks far away from the origin is larger than that close to the origin. This behavior can be understood from Fig:1(b). The resonant points at high energies are close to each other. Thus, a small difference of the dynamical gap can make them cross successively the bias windows, while for the resonant points at the low energy, a larger one is required to shift them for the occurrence of the resonant tunneling. Fig.3(b) shows the dependence of the differential conductance on the radiation frequency $\omega_{0}$ for different radiation intensities in the parallel electrodes configuration. It is clearly seen that when $\omega_{0}$ approaches to zero, the differential conductance tends to vanish owing 


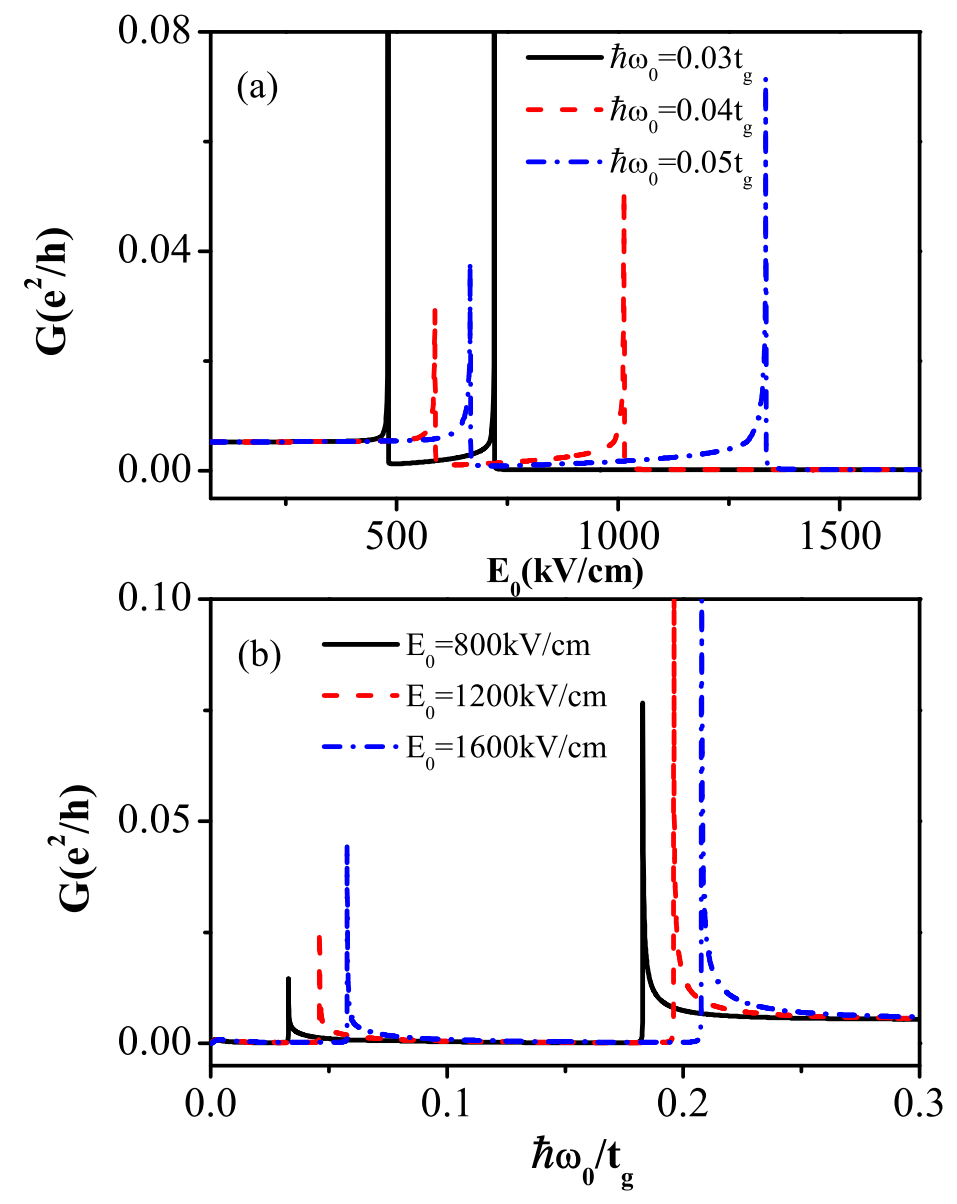

Figure 3. (color online)The differential conductance $G$ as a function of the radiation field strength for different frequencies in (a), and as a function of the frequency for different radiation strengths in (b). In both cases we consider the parallel configuration of the electrodes' magnetizations at $\mathrm{eV}=0.15 \mathrm{t}_{\mathrm{g}}$. The other parameters are taken the same as those of Fig 1 .

to the large dynamical gap. With increasing the frequency, the dynamical gap shrinks, which causes the shift of the photon-induced subbands, therefore, one can observe that two resonant peaks emerge successively because of the bias windows crossing of the subbands which is induced by the external radiation field. Additionally, the increase of the radiation intensity enlarges the dynamical gap, thus shifting the resonant peaks in the differential conductance in the direction away from the origin. The amount of the peaks shift at high frequencies is the same as at low frequencies. This is because when changing the radiation strength, the interval of the resonant points at high energies is equivalent to that at the low energies (see Fig.1(a)). Thus the same variation of the radiation intensity is needed to achieve the crossing of the bias windows due to the linear dependence of the dynamical gap on the radiation field.

The bias dependence of the TMR for the different radiation strengths and frequencies is shown in Fig. 4. The TMR as a function of the bias voltage exhibits a sharp peak which is caused by photon-assisted effects, and a plateau around the zero 


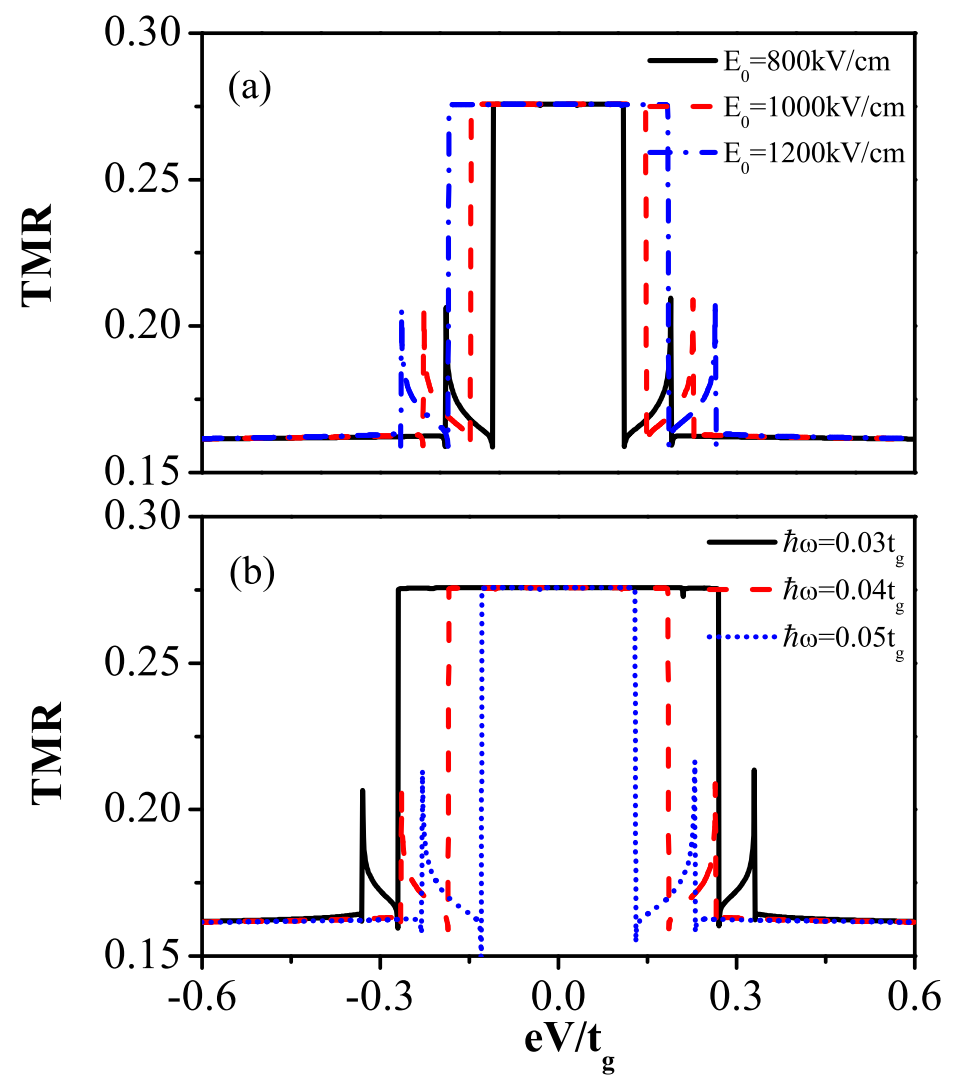

Figure 4. (color online) The TMR versus the bias voltage for different radiation field strength $E_{0}$ at $\hbar \omega_{0}=0.04 t_{g}(\mathrm{a})$, and for different radiation frequencies $\omega_{0}$ at $E_{0}=1200$ $\mathrm{kV} / \mathrm{cm}$ in (b). The other parameters are taken the same as those of Fig 1

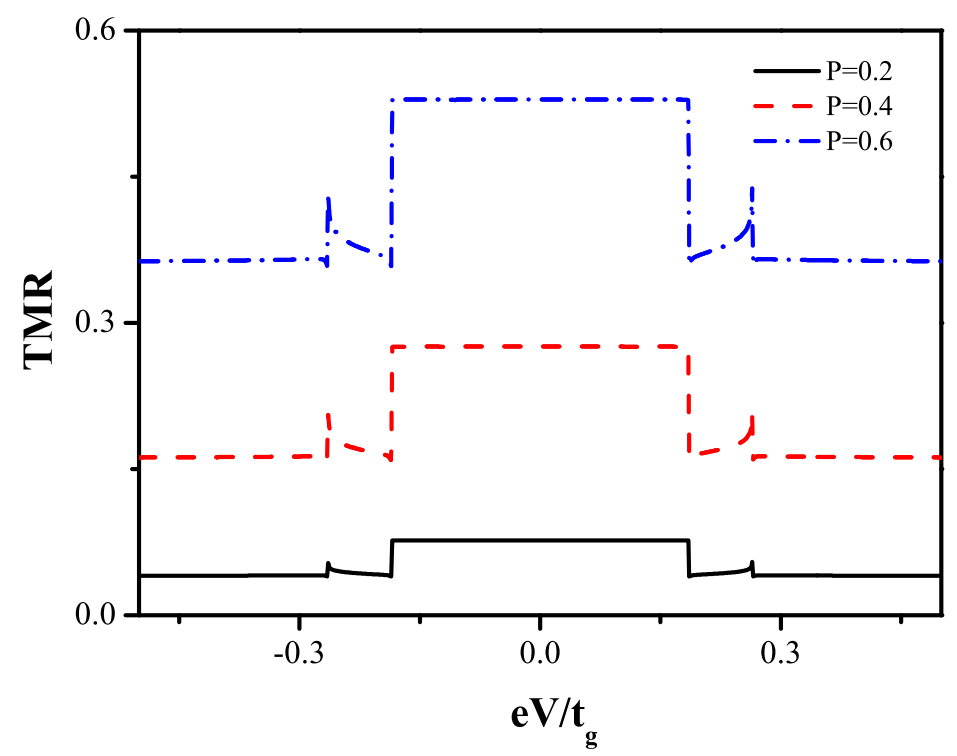

Figure 5. (color online) The TMR versus the bias voltage for a different polarization $p$ at $\hbar \omega_{0}=0.04 t_{g}$ and $E_{0}=1200 \mathrm{kV} / \mathrm{cm}$. The other parameters are taken the same as those of Fig 1 . 
bias voltage. We assign this behavior to the combined effects of the radiation field, the graphene, and the spin valve properties. In order to further clarify this point, we plot the bias dependence of the TMR for different polarizations in Fig. 5. One can observe that the TMR changes in a nonlinear manner, i.e. the TMR values near zero bias voltage become larger than those in the high bias regions. This is because graphene remains insulator-like in the gap regions, the ballistic spin tunneling enhances the TMR. Remarkablely, the width of the plateau near the zero bias voltage can be tuned by the radiation intensity and frequency(see Fig. 4). With increasing the radiation intensity (or frequency), the width of the plateau increases (or decreases), which is also related to the dynamical gap induced by the radiation field.

\section{Summary}

In conclusion, we studied theoretically the spin polarized transport through a graphene spin valve device assisted by a linearly polarized, monochromatic laser field and in the presence of a dc bias and a gate voltage. The method is based on the standard Keldysh nonequilibrium Green's function approach. We find that the bias dependence of the differential conductance exhibits two resonant peaks due to the resonant tunneling through the photon-assisted subbands. The resonant interaction of the quasiparticle in graphene with the radiation field turns on a dynamical gap in the quasiparticle spectrum. When the bias voltage lies inside this gap region, the differential conductance displays a zero value plateau situated symmetrically to the zero bias. The value of the dynamical gap depends on the strength and frequency of the external radiation field, thus the width of this zero value plateau in the differential conductance can be tuned by changing the radiation intensity and frequency, which also causes a shift of the resonant peaks in the differential conductance. We explored the behavior of the peaks in the differential conductance with varying radiation field strength and frequency and revealed the dependence on the spin polarization of the ferromagnetic electrodes. We also demonstrated that the combined effects of the radiation field, the graphene, and the spin valve properties bring about a symmetric plateau at zero bias voltage in the TMR. The width of this plateau can be varied by changing the parameters of the radiation field.

\section{Acknowledgments}

The work of K.H.D. is supported by DAAD (Germany) and by the National Natural Science Foundation of China (Grant Nos. 10904007), and the construct program of the key discipline in Hunan Province, China. 


\section{Appendix A.}

In this appendix, we present the analytical results of the Green's function $g_{s s^{\prime}}^{\tau \tau^{\prime}, r}(t)$ for the graphene without the coupling to the electrodes in the absence of the gate voltage. From the Hamiltonian $H_{G}$, we find by using the equation of motion method that

$$
g_{s s^{\prime}}^{\tau \tau, r}(t)=\int \frac{d \varepsilon}{2 \pi} g_{s s^{\prime}}^{\tau \tau, r}(\varepsilon) e^{-i \varepsilon t}
$$

where $g^{\tau \tau, r}(\varepsilon)=\frac{1}{N} \sum_{\mathbf{k}} g_{\mathbf{k}}^{\tau \tau, r}(\varepsilon)$ with

$$
g_{\mathbf{k}}^{\tau \tau, r}(\varepsilon)=\left(\begin{array}{ll}
\frac{\varepsilon-\widetilde{\epsilon}_{-\mathbf{k}}}{\left(\varepsilon-\widetilde{\epsilon}_{+\mathbf{k}}\right)\left(\varepsilon-\widetilde{\epsilon}_{-\mathbf{k}}\right)-\Delta^{2}} & \frac{\Delta}{\left(\varepsilon-\widetilde{\epsilon}_{+\mathbf{k}}\right)\left(\varepsilon-\widetilde{\epsilon}_{-\mathbf{k}}\right)-\Delta^{2}} \\
\frac{\left.\varepsilon-\widetilde{\epsilon}_{+\mathbf{k}}\right)}{\left(\varepsilon-\widetilde{\epsilon}_{+\mathbf{k}}\right)\left(\varepsilon-\widetilde{\epsilon}_{-\mathbf{k}}\right)-\Delta^{2}} & \frac{\left.\widetilde{c}_{+\mathbf{k}}\right)\left(\varepsilon-\widetilde{\epsilon}_{-\mathbf{k}}\right)-\Delta^{2}}{\left(\varepsilon-\widetilde{\epsilon}_{+}\right.}
\end{array}\right)
$$

To solve $g_{s s^{\prime}}^{\tau \tau, r}(\varepsilon)$, we need to convert the summation over $\mathbf{k}$ into a integral in two dimensional momentum space. After a straightforward calculation, we have for $|\varepsilon|<\Delta$,

$$
\begin{aligned}
g_{++}^{\tau \tau, r}= & -\frac{1}{2 \pi \rho v_{F}^{2}}\left\{\left(\frac{\varepsilon}{2}+\frac{\omega_{0}}{4}\right) \ln \left|\frac{\left(D-\omega_{0} / 2\right)^{2}+\Delta^{2}-\varepsilon^{2}}{\omega_{0}^{2} / 4+\Delta^{2}-\varepsilon^{2}}\right|\right. \\
& \left.-\frac{1}{\sqrt{\Delta^{2}-\varepsilon^{2}}}\left(\Delta^{2}-\varepsilon^{2}-\frac{\omega_{0}}{2} \varepsilon\right)\left[\arctan \frac{D-\omega_{0} / 2}{\sqrt{\Delta^{2}-\varepsilon^{2}}}-\arctan \frac{-\omega_{0} / 2}{\sqrt{\Delta^{2}-\varepsilon^{2}}}\right]+D\right\} \\
g_{--}^{\tau \tau, r}= & -\frac{1}{2 \pi \rho v_{F}^{2}}\left\{\left(\frac{\varepsilon}{2}-\frac{\omega_{0}}{4}\right) \ln \left|\frac{\left(D-\omega_{0} / 2\right)^{2}+\Delta^{2}-\varepsilon^{2}}{\omega_{0}^{2} / 4+\Delta^{2}-\varepsilon^{2}}\right|\right. \\
& \left.+\frac{\Delta^{2}-\varepsilon^{2}+\varepsilon \omega_{0} / 2}{\sqrt{\Delta^{2}-\varepsilon^{2}}}\left[\arctan \frac{D-\omega_{0} / 2}{\sqrt{\Delta^{2}-\varepsilon^{2}}}-\arctan \frac{-\omega_{0} / 2}{\sqrt{\Delta^{2}-\varepsilon^{2}}}\right]-D\right\} \\
g_{+-}^{\tau \tau, r}=g_{-+}^{\tau \tau, r}= & -\frac{1}{2 \pi \rho v_{F}^{2}}\left\{\frac{\Delta}{2} \ln \left|\frac{\left(D-\omega_{0} / 2\right)^{2}+\Delta^{2}-\varepsilon^{2}}{\omega_{0}^{2} / 4+\Delta^{2}-\varepsilon^{2}}\right|\right. \\
& \left.+\frac{\Delta \omega_{0} / 2}{\sqrt{\Delta^{2}-\varepsilon^{2}}}\left[\arctan \frac{D-\omega_{0} / 2}{\sqrt{\Delta^{2}-\varepsilon^{2}}}-\arctan \frac{-\omega_{0} / 2}{\sqrt{\Delta^{2}-\varepsilon^{2}}}\right]\right\}
\end{aligned}
$$

For $|\varepsilon|>\Delta$

$$
\begin{aligned}
& g_{++}^{\tau \tau, r}=-\frac{1}{4 \pi \rho v_{F}^{2}}\left[\left(\varepsilon+\frac{\omega_{0}}{2}\right) \ln \left|\frac{\left(D-\omega_{0} / 2\right)^{2}-\varepsilon^{2}+\Delta^{2}}{\varepsilon^{2}-\Delta^{2}-\omega_{0}^{2} / 4}\right|\right. \\
& \left.-\left(\varepsilon \frac{\frac{\omega_{0}}{2}}{\sqrt{\varepsilon^{2}-\Delta^{2}}}+\sqrt{\varepsilon^{2}-\Delta^{2}}\right) \ln \left|\frac{\left(D-\omega_{0} / 2+\sqrt{\varepsilon^{2}-\Delta^{2}}\right)\left(\omega_{0} / 2+\sqrt{\varepsilon^{2}-\Delta^{2}}\right)}{\left(D-\omega_{0} / 2-\sqrt{\varepsilon^{2}-\Delta^{2}}\right)\left(\omega_{0} / 2-\sqrt{\varepsilon^{2}-\Delta^{2}}\right)}\right|+2 D\right] \\
& +\frac{1}{4 \pi \rho v_{F}^{2}} i \pi \operatorname{sgn}(\varepsilon)\left\{\theta\left(\Delta<|\varepsilon|<\sqrt{\frac{\omega_{0}^{2}}{4}+\Delta^{2}}\right)\left(\varepsilon-\sqrt{\varepsilon^{2}-\Delta^{2}}\right)\left(1-\frac{\frac{\omega_{0}}{2}}{\sqrt{\varepsilon^{2}-\Delta^{2}}}\right)\right. \\
& \left.-\theta\left(\Delta<|\varepsilon|<\sqrt{\left(D-\frac{\omega_{0}}{2}\right)^{2}+\Delta^{2}}\right)\left(\varepsilon+\sqrt{\varepsilon^{2}-\Delta^{2}}\right)\left(1+\frac{\frac{\omega_{0}}{2}}{\sqrt{\varepsilon^{2}-\Delta^{2}}}\right)\right\} \\
& g_{--}^{\tau \tau, r}=-\frac{1}{4 \pi \rho v_{F}^{2}}\left[\left(\varepsilon-\frac{\omega_{0}}{2}\right) \ln \left|\frac{\left(D-\omega_{0} / 2\right)^{2}-\varepsilon^{2}+\Delta^{2}}{\varepsilon^{2}-\Delta^{2}-\omega_{0}^{2} / 4}\right|\right. \\
& \left.+\left(\sqrt{\varepsilon^{2}-\Delta^{2}}-\varepsilon \frac{\frac{\omega_{0}}{2}}{\sqrt{\varepsilon^{2}-\Delta^{2}}}\right) \ln \left|\frac{\left(D-\omega_{0} / 2+\sqrt{\varepsilon^{2}-\Delta^{2}}\right)\left(\omega_{0} / 2+\sqrt{\varepsilon^{2}-\Delta^{2}}\right)}{\left(D-\omega_{0} / 2-\sqrt{\varepsilon^{2}-\Delta^{2}}\right)\left(\omega_{0} / 2-\sqrt{\varepsilon^{2}-\Delta^{2}}\right)}\right|-2 D\right] \\
& +\frac{1}{4 \pi \rho v_{F}^{2}} i \pi \operatorname{sgn}(\varepsilon)\left\{\theta\left(\Delta<|\varepsilon|<\sqrt{\frac{\omega_{0}^{2}}{4}+\Delta^{2}}\right)\left(\varepsilon+\sqrt{\varepsilon^{2}-\Delta^{2}}\right)\left(1-\frac{\frac{\omega_{0}}{2}}{\sqrt{\varepsilon^{2}-\Delta^{2}}}\right)\right. \\
& \left.-\theta\left(\Delta<|\varepsilon|<\sqrt{\left(D-\frac{\omega_{0}}{2}\right)^{2}+\Delta^{2}}\right)\left(\varepsilon-\sqrt{\varepsilon^{2}-\Delta^{2}}\right)\left(1+\frac{\frac{\omega_{0}}{2}}{\sqrt{\varepsilon^{2}-\Delta^{2}}}\right)\right\} \\
& g_{+-}^{\tau \tau, r}=g_{-+}^{\tau \tau, r} \\
& =-\frac{\Delta}{4 \pi \rho v_{F}^{2}}\left[\ln \left|\frac{\left(D-\omega_{0} / 2\right)^{2}-\varepsilon^{2}+\Delta^{2}}{\varepsilon^{2}-\Delta^{2}-\omega_{0}^{2} / 4}\right|-\frac{\frac{\omega_{0}}{2}}{\sqrt{\varepsilon^{2}-\Delta^{2}}} \ln \left|\frac{\left(D-\omega_{0} / 2+\sqrt{\varepsilon^{2}-\Delta^{2}}\right)\left(\omega_{0} / 2+\sqrt{\varepsilon^{2}-\Delta^{2}}\right)}{\left(D-\omega_{0} / 2-\sqrt{\varepsilon^{2}-\Delta^{2}}\right)\left(\omega_{0} / 2-\sqrt{\varepsilon^{2}-\Delta^{2}}\right)}\right|\right] \\
& +\frac{\Delta}{4 \pi \rho v_{F}^{2}} i \pi \operatorname{sgn}(\varepsilon)\left\{\theta\left(\Delta<|\varepsilon|<\sqrt{\frac{\omega_{0}^{2}}{4}+\Delta^{2}}\right)\left(1-\frac{\frac{\omega_{0}}{2}}{\sqrt{\varepsilon^{2}-\Delta^{2}}}\right)\right. \\
& \left.-\theta\left(\Delta<|\varepsilon|<\sqrt{\left(D-\frac{\omega_{0}}{2}\right)^{2}+\Delta^{2}}\right)\left(1+\frac{\frac{\omega_{0}}{2}}{\sqrt{\varepsilon^{2}-\Delta^{2}}}\right)\right\}
\end{aligned}
$$

where $\rho$ is the graphene planar density, $D$ is a high-energy cutoff of the graphene bandwidth. When the gate voltage is taken into account, the Green's functions for the irradiated graphene are obtained by changing $\varepsilon$ to $\varepsilon-V_{g}$ in equations (A.3)-(A.8). 
[1] X. Duan, Y. Huang, Y. Cui, J. Wang, and C. M. Lieber, Nature 409, 66 (2001).

[2] M. S. Marcus, J. M. Simmons, O. M. Castellini, R. J. Hammers, and M. A. Eriksson, J. Appl. Phys. 100, 084306 (2006).

[3] M. Freitag, Y. Martin, J. A. Misewich, R. Martel, and P. Avouris, Nano Lett. 3, 1067 (2003).

[4] Y. Ohno, S. Kishimoto, T. Mizutani, T. Okazaki, and H. Shinohara, Appl. Phys. Lett. 84, 1368 (2004). 25

[5] J. Wang, M. S. Gudiksen, X. Duan, Y. Cui, and C. M. Lieber, Science 293, 1455 (2001).

[6] K. S. Novoselov, A. K. Geim, S. V. Morozov, D. Jiang, M. I. Katsnelson, I. V. Grigorieva, S. V. Dubonos, and A. A. Firsov, Nature 438, 197 (2005).

[7] M. I. Katsnelson, K. S. Novoselov, and A. K. Geim, Nat. Phys. 2, 620 (2006).

[8] A. K. Geim and K. S. Novoselov, Nature Materials 6, 183 (2007).

[9] N. M. R. Peres, F. Guinea, and A. H. Castro Neto, Phys. Rev. B 73, 125411(2006).

[10] L. A. Falkovsky and A. A. Varlamov, Europ. Phys. J. B 56, 281 (2007).

[11] V. P. Gusynin and S. G. Sharapov, Phys. Rev. B 73, 245411 (2006).

[12] B. Trauzettel, Y. M. Blanter, and A. F. Morpurgo, Phys. Rev. B 75, 035305 (2007).

[13] K. H. Ding, Z. -G. Zhu, and J. Berakdar, Phys. Rev. B 84, 115433 (2011).

[14] V. P. Gusynin, S. G. Sharapov, and J. P. Carbotte, Phys. Rev. B 75, 165407 (2007).

[15] L. A. Falkovsky and S. S. Pershoguba, Phys. Rev. B 76, 153410 (2007).

[16] D. S. L. Abergel and V. I. Falko, Phys. Rev. B 75, 155430 (2007).

[17] E. H. Hwang and S. Das Sarma, Phys. Rev. B 75, 205418 (2007)

[18] B. Wunsch, T. Stauber, F. Sols, and F. Guinea, New J. Phys. 8, 318 (2006).

[19] V. Apalkov, X.-F. Wang, and T. Chakraborty, Int. J. Mod. Phys. B 21, 1165 (2007).

[20] O. Vafek, Phys. Rev. Lett. 97, 266406 (2006).

[21] S. A. Mikhailov and K. Ziegler, Phys. Rev. Lett. 99, 016803 (2007).

[22] S. A. Mikhailov and K. Ziegler, J. Phys.: Condens. Matter 20, 384204(2008)

[23] A. S. Moskalenko, J. Berakdar, Phys. Rev. B 80, 193407 (2009).

[24] B. Sensale-Rodriguez, T. Fang, R. Yan, M. M. Kelly, D. Jena, L. Liu, and H. Xing Appl. Phys. Lett. 99, 113104 (2011); F. Rana, IEEE Trans. Nanotechnol. 7, 91 (2008); V. Ryzhii, M. Ryzhii, V. Mitin, and T. Otsuji, J. Appl. Phys. 107, 054512 (2010).

[25] M. V. Fistul and K. B. Efetov, Phys. Rev. Lett. 98, 256803 (2007)

[26] S. V. Syzranov, M. V. Fistul, and K. B. Efetov, Phys. Rev. B 78, 045407 (2008)

[27] E. W. Hill, A. K. Geim, K. Novoselov, F. Schedin, and P. Black, IEEE Trans. Magn. 42, 2694 (2006).

[28] N. Tombros, C. Jozsa, M. Popinciue, H. T. Jonkman, and B. J. Van Wees, Nature 448, 571 (2007).

[29] M. Ohishi, M. Shiraishi, R. Nouchi, T. Nozaki, T. Shinjo, and Y. Suzuki, Jap. J. Appl. Phys. 46, L605 (2007).

[30] S. Cho, Yung-Fu Chen, and M. S. Fuhrer, Appl. Phys. Lett. 91, 123105 (2007).

[31] W. H. Wang, K. Pi, Y. Li, Y. F. Chiang, P. Wei, J. Shi, and R. K. Kawakami, Phys. Rev. B 77, 020402(R) (2008).

[32] K. H. Ding, Z.-G. Zhu, and J. Berakdar, Phys. Rev. B 79, 045405 (2009); EPL 88, 58001 (2009); J. Phys.: Condensed Matt. 20, 345228 (2008).

[33] J. Maassen, W. Ji, H. Guo, Nano Lett. 11, 151 (2011).

[34] J. -C. Chen, S. -G. Cheng, S. -Q. Shen, and Q. -F. Sun, J. Phys.: Condens. Matter 22, 035301 (2010)

[35] K. H. Ding, Z. -G. Zhu, Z.-H. Zhang, and J. Berakdar, Phys. Rev. B 82, 155143 (2010).

[36] X. Zhang, J. Wang, and S. C. Zhang, Phys. Rev. B 82, 245107 (2010).

[37] H. Haug and A. P. Jauho, Quantum Kinetics in Transport and Optics of Semiconductors (Springer, Berlin, 1998).

[38] J. Rammer, Quantum Transport Theory (Westview Press, Boulder, CO, 2004).

[39] A. H. Castro Neto, F. Guinea, N. M. R. Peres, K. S. Novoselov, A. K. Geim, Rev. Mod. Phys. 81, 109 (2009). 
[40] C. C. Gerry, P. L. Knight, Introductory Quantum Optics, Cambridge University Press, Cambridge, 2005.

[41] A. Levy Yeyati, and F. Flores, Phys. Rev. B 44, 9020 (1991). 
Laser-assisted spin-polarized transport in graphene tunnel junctions

\section{Supplementary materials}

\section{Appendix B. Rotating wave approximation}

Here we give full details of the rotating wave approximation to the Hamiltonian $H_{G}$ as defined in the main text, i.e.

$$
\begin{aligned}
& H_{G}=H_{0}+H_{I}=\sum_{\mathbf{k} s \tau} \epsilon_{s \mathbf{k}} a_{s \mathbf{k} \tau}^{\dagger} a_{s \mathbf{k} \tau}-e v_{F} A(t) \sum_{\tau} \sum_{\mathbf{k} s s^{\prime}} d_{s s^{\prime}, \mathbf{k}} a_{s \mathbf{k} \tau}^{\dagger} a_{s^{\prime} \mathbf{k} \tau} \\
& H_{0}=\sum_{\mathbf{k} \tau}\left[\epsilon_{+\mathbf{k}} a_{+\mathbf{k} \tau}^{\dagger} a_{+\mathbf{k} \tau}+\epsilon_{-\mathbf{k}} a_{-\mathbf{k} \tau}^{\dagger} a_{-\mathbf{k} \tau}\right] \\
& H_{I}=-e v_{F} A(t) \sum_{\mathbf{k} \tau}\left[d_{++, \mathbf{k}} a_{+\mathbf{k} \tau}^{\dagger} a_{+\mathbf{k} \tau}+d_{--, \mathbf{k}} a_{-\mathbf{k} \tau}^{\dagger} a_{-\mathbf{k} \tau}+d_{+-, \mathbf{k}} a_{+\mathbf{k} \tau}^{\dagger} a_{-\mathbf{k} \tau}+d_{-+, \mathbf{k}} a_{-\mathbf{k} \tau}^{\dagger} a_{+\mathbf{k} \tau}\right]
\end{aligned}
$$

where $A(t)=A_{0} e^{i \omega_{0} t}+A_{0}^{*} e^{-i \omega_{0}}$. In the interaction picture, we have

$$
\bar{H}=i \dot{U} U^{\dagger}+U H_{G} U^{\dagger}=U H_{I} U^{\dagger}=e^{i H_{0} t} H_{I} e^{-i H_{0} t}
$$

Using the formula

$$
e^{A} B e^{-A}=\sum_{n=0}^{\infty} \frac{1}{n !} C_{n}, \quad C_{n}=\left[A, C_{n-1}\right], \quad C_{0}=B
$$

we have

$$
\begin{aligned}
& {\left[i t H_{0}, H_{I}\right]=} \\
& \quad-\sum_{\mathbf{k} \tau \mathbf{k}^{\prime} \tau^{\prime}} i t e v_{F} A(t)\left\{\epsilon_{+\mathbf{k}} d_{+-, \mathbf{k}^{\prime}}\left[a_{+\mathbf{k} \tau}^{\dagger} a_{+\mathbf{k} \tau}, a_{+\mathbf{k}^{\prime} \tau^{\prime}}^{\dagger} a_{-\mathbf{k}^{\prime} \tau^{\prime}}\right]+\epsilon_{-\mathbf{k}} d_{+-, \mathbf{k}^{\prime}}\left[a_{-\mathbf{k} \tau}^{\dagger} a_{-\mathbf{k} \tau}, a_{+\mathbf{k}^{\prime} \tau^{\prime}}^{\dagger} a_{-\mathbf{k}^{\prime} \tau^{\prime}}\right]\right. \\
& \left.\quad+\epsilon_{+\mathbf{k}} d_{-+, \mathbf{k}^{\prime}}\left[a_{+\mathbf{k} \tau}^{\dagger} a_{+\mathbf{k} \tau}, a_{-\mathbf{k}^{\prime} \tau^{\prime}}^{\dagger} a_{+\mathbf{k}^{\prime} \tau^{\prime}}\right]+\epsilon_{-\mathbf{k}} d_{-+, \mathbf{k}^{\prime}}\left[a_{-\mathbf{k} \tau}^{\dagger} a_{-\mathbf{k} \tau}, a_{-\mathbf{k}^{\prime} \tau^{\prime}}^{\dagger} a_{+\mathbf{k}^{\prime} \tau^{\prime}}\right]\right\} \\
& =-\sum_{\mathbf{k} \tau \mathbf{k}^{\prime} \tau^{\prime}} i t e v_{F} A(t)\left\{\epsilon_{+\mathbf{k}} d_{+-, \mathbf{k}^{\prime}}\left(a_{+\mathbf{k} \tau}^{\dagger}\left[a_{+\mathbf{k} \tau}, a_{+\mathbf{k}^{\prime} \tau^{\prime}}^{\dagger} a_{-\mathbf{k}^{\prime} \tau^{\prime}}\right]+\left[a_{+\mathbf{k} \tau}^{\dagger}, a_{+\mathbf{k}^{\prime} \tau^{\prime}}^{\dagger} a_{-\mathbf{k}^{\prime} \tau^{\prime}}\right] a_{+\mathbf{k} \tau}\right)\right. \\
& \quad+\epsilon_{-\mathbf{k}} d_{+-, \mathbf{k}^{\prime}}\left(a_{-\mathbf{k} \tau}^{\dagger}\left[a_{-\mathbf{k} \tau}, a_{+\mathbf{k}^{\prime} \tau^{\prime}}^{\dagger} a_{-\mathbf{k}^{\prime} \tau^{\prime}}\right]+\left[a_{-\mathbf{k} \tau}^{\dagger}, a_{+\mathbf{k}^{\prime} \tau^{\prime}}^{\dagger} a_{-\mathbf{k}^{\prime} \tau^{\prime}}\right] a_{-\mathbf{k} \tau}\right) \\
& \quad+\epsilon_{+\mathbf{k}} d_{-+, \mathbf{k}^{\prime}}\left(a_{+\mathbf{k} \tau}^{\dagger}\left[a_{+\mathbf{k} \tau}, a_{-\mathbf{k}^{\prime} \tau^{\prime}}^{\dagger} a_{+\mathbf{k}^{\prime} \tau^{\prime}}\right]+\left[a_{+\mathbf{k} \tau}^{\dagger}, a_{-\mathbf{k}^{\prime} \tau^{\prime}}^{\dagger} a_{+\mathbf{k}^{\prime} \tau^{\prime}}\right] a_{+\mathbf{k} \tau}\right) \\
& \left.\quad+\epsilon_{-\mathbf{k}} d_{-+, \mathbf{k}^{\prime}}\left(a_{-\mathbf{k} \tau}^{\dagger}\left[a_{-\mathbf{k} \tau}, a_{-\mathbf{k}^{\prime} \tau^{\prime}}^{\dagger} a_{+\mathbf{k}^{\prime} \tau^{\prime}}\right]+\left[a_{-\mathbf{k} \tau}^{\dagger}, a_{-\mathbf{k}^{\prime} \tau^{\prime}}^{\dagger} a_{+\mathbf{k}^{\prime} \tau^{\prime}}\right] a_{-\mathbf{k} \tau}\right)\right\} \\
& =-\sum_{\mathbf{k} \tau \mathbf{k}^{\prime} \tau^{\prime}} i t e v_{F} A(t)\left\{\epsilon_{+\mathbf{k}} d_{+-, \mathbf{k}^{\prime}} \delta_{\mathbf{k} \mathbf{k}^{\prime}} \delta_{\tau \tau^{\prime}} a_{+\mathbf{k} \tau}^{\dagger} a_{-\mathbf{k}^{\prime} \tau^{\prime}}-\epsilon_{-\mathbf{k}} d_{+-, \mathbf{k}^{\prime}} \delta_{\mathbf{k} \mathbf{k}^{\prime}} \delta_{\tau \tau^{\prime}} a_{+\mathbf{k}^{\prime} \tau^{\prime}}^{\dagger} a_{-\mathbf{k} \tau}\right. \\
& \left.\quad-\epsilon_{+\mathbf{k}} d_{-+, \mathbf{k}^{\prime}} \delta_{\mathbf{k} \mathbf{k}^{\prime}} \delta_{\tau \tau^{\prime}} a_{-\mathbf{k}^{\prime} \tau^{\prime}}^{\dagger} a_{+\mathbf{k} \tau}+\epsilon_{-\mathbf{k}} d_{-+, \mathbf{k}^{\prime}} \delta_{\mathbf{k} \mathbf{k}^{\prime}} \delta_{\tau \tau^{\prime}} a_{-\mathbf{k} \tau}^{\dagger} a_{+\mathbf{k}^{\prime} \tau^{\prime}}\right\}
\end{aligned}
$$

which means that

$$
\begin{aligned}
{\left[i t H_{0}, H_{I}\right]=} & -\sum_{\mathbf{k} \tau} \operatorname{itev}_{F} A(t)\left\{\epsilon_{+\mathbf{k}} d_{+-, \mathbf{k}} a_{+\mathbf{k} \tau}^{\dagger} a_{-\mathbf{k} \tau}-\epsilon_{-\mathbf{k}} d_{+-, \mathbf{k}} a_{+\mathbf{k} \tau}^{\dagger} a_{-\mathbf{k} \tau}\right. \\
& \left.-\epsilon_{+\mathbf{k}} d_{-+, \mathbf{k}} a_{-\mathbf{k} \tau}^{\dagger} a_{+\mathbf{k} \tau}+\epsilon_{-\mathbf{k}} d_{-+, \mathbf{k}} a_{-\mathbf{k} \tau}^{\dagger} a_{+\mathbf{k} \tau}\right\} \\
= & -\sum_{\mathbf{k} \tau} i t e v_{F} A(t)\left\{\left(\epsilon_{+\mathbf{k}}-\epsilon_{-\mathbf{k}}\right) d_{+-, \mathbf{k}} a_{+\mathbf{k} \tau}^{\dagger} a_{-\mathbf{k} \tau}-\left(\epsilon_{+\mathbf{k}}-\epsilon_{-\mathbf{k}}\right) d_{-+, \mathbf{k}} a_{-\mathbf{k} \tau}^{\dagger} a_{+\mathbf{k} \tau}\right\}
\end{aligned}
$$


thus

$$
\begin{gathered}
\bar{H}=H_{I}-e v_{F} A(t) \sum_{\mathbf{k} \tau}\left\{i t\left(\epsilon_{+\mathbf{k}}-\epsilon_{-\mathbf{k}}\right) d_{+-, \mathbf{k}} a_{+\mathbf{k} \tau}^{\dagger} a_{-\mathbf{k} \tau}-i t\left(\epsilon_{+\mathbf{k}}-\epsilon_{-\mathbf{k}}\right) d_{-+, \mathbf{k}} a_{-\mathbf{k} \tau}^{\dagger} a_{+\mathbf{k} \tau}\right\} \\
-e v_{F} A(t) \sum_{\mathbf{k} \tau}\left\{\frac{1}{2 !}\left[i t\left(\epsilon_{+\mathbf{k}}-\epsilon_{-\mathbf{k}}\right)\right]^{2} d_{+-, \mathbf{k}} a_{+\mathbf{k} \tau}^{\dagger} a_{-\mathbf{k} \tau}+\frac{1}{2 !}\left[-i t\left(\epsilon_{+\mathbf{k}}-\epsilon_{-\mathbf{k}}\right)\right]^{2} d_{-+, \mathbf{k}} a_{-\mathbf{k} \tau}^{\dagger} a_{+\mathbf{k} \tau}\right\}+\cdots \\
-e v_{F} A(t) \sum_{\mathbf{k} \tau}\left\{\frac{1}{n !}\left[i t\left(\epsilon_{+\mathbf{k}}-\epsilon_{-\mathbf{k}}\right)\right]^{n} d_{+-, \mathbf{k}} a_{+\mathbf{k} \tau}^{\dagger} a_{-\mathbf{k} \tau}+\frac{1}{n !}\left[-i t\left(\epsilon_{+\mathbf{k}}-\epsilon_{-\mathbf{k}}\right)\right]^{n} d_{-+, \mathbf{k}} a_{-\mathbf{k} \tau}^{\dagger} a_{+\mathbf{k} \tau}\right\}+\cdots \\
=-e v_{F} A(t) \sum_{\mathbf{k} \tau}\left(d_{++, \mathbf{k}} a_{+\mathbf{k} \tau}^{\dagger} a_{+\mathbf{k} \tau}+d_{--, \mathbf{k}} a_{-\mathbf{k} \tau}^{\dagger} a_{-\mathbf{k} \tau}\right) \\
-e v_{F} A(t) \sum_{\mathbf{k} \tau}\left\{\left\{1+i t\left(\epsilon_{+\mathbf{k}}-\epsilon_{-\mathbf{k}}\right)+\frac{1}{2 !}\left[i t\left(\epsilon_{+\mathbf{k}}-\epsilon_{-\mathbf{k}}\right)\right]^{2}+\cdots\right\} d_{+-, \mathbf{k}} a_{+\mathbf{k} \tau}^{\dagger} a_{-\mathbf{k} \tau}\right. \\
\left.+\left\{1-i t\left(\epsilon_{+\mathbf{k}}-\epsilon_{-\mathbf{k}}\right)+\frac{1}{2 !}\left[-i t\left(\epsilon_{+\mathbf{k}}-\epsilon_{-\mathbf{k}}\right)\right]^{2}+\cdots\right\} d_{-+, \mathbf{k}} a_{-\mathbf{k} \tau}^{\dagger} a_{+\mathbf{k} \tau}\right\} \\
=-e v_{F} A(t) \sum_{\mathbf{k} \tau}\left(d_{++, \mathbf{k}} a_{+\mathbf{k} \tau}^{\dagger} a_{+\mathbf{k} \tau}+d_{--, \mathbf{k}} a_{-\mathbf{k} \tau}^{\dagger} a_{-\mathbf{k} \tau}\right) \\
-e v_{F} A(t) \sum_{\mathbf{k} \tau}\left\{e^{i t\left(\epsilon_{+\mathbf{k}}-\epsilon_{-\mathbf{k}}\right)} d_{+-, \mathbf{k}} a_{+\mathbf{k} \tau}^{\dagger} a_{-\mathbf{k} \tau}+e^{-i t\left(\epsilon_{+\mathbf{k}}-\epsilon_{-\mathbf{k}}\right)} d_{-+, \mathbf{k}} a_{-\mathbf{k} \tau}^{\dagger} a_{+\mathbf{k} \tau}\right\}
\end{gathered}
$$

Under the rotating wave approximation,Eq.(B.7) can be approximated as

$$
\bar{H}=-e v_{F} \sum_{\mathbf{k} \tau}\left\{A_{0}^{*} e^{i t\left(\epsilon_{+\mathbf{k}}-\epsilon_{-\mathbf{k}}-\omega_{0}\right)} d_{+-, \mathbf{k}} a_{+\mathbf{k} \tau}^{\dagger} a_{-\mathbf{k} \tau}+A_{0} e^{-i t\left(\epsilon_{+\mathbf{k}}-\epsilon_{-\mathbf{k}}-\omega_{0}\right)} d_{-+, \mathbf{k}} a_{-\mathbf{k} \tau}^{\dagger} a_{+\mathbf{k} \tau}\right\} .
$$

The next step is to transform the approximate Hamiltonian back to the Schrödinger picture:

$$
H_{I}=e^{-i H_{0} t} H_{I} e^{i H_{0} t}=-e v_{F} \sum_{\mathbf{k} \tau}\left\{A_{0}^{*} e^{-i \omega_{0} t} d_{+-, \mathbf{k}} a_{+\mathbf{k} \tau}^{\dagger} a_{-\mathbf{k} \tau}+A_{0} e^{i \omega_{0} t} d_{-+, \mathbf{k}} a_{-\mathbf{k} \tau}^{\dagger} a_{+\mathbf{k} \tau}\right\}
$$

\section{Appendix C. Electric current formula}

The Hamiltonian $H=H_{G}+H_{L}+H_{R}+H_{T}$ is determined by

$$
\begin{aligned}
& H_{G}=\sum_{\mathbf{k} s \tau} \epsilon_{s \mathbf{k}} a_{s \mathbf{k} \tau}^{\dagger} a_{s \mathbf{k} \tau}-e v_{F} \sum_{\mathbf{k} \tau}\left\{A_{0}^{*} e^{-i \omega_{0} t} d_{+-, \mathbf{k}} a_{+\mathbf{k} \tau}^{\dagger} a_{-\mathbf{k} \tau}+A_{0} e^{i \omega_{0} t} d_{-+, \mathbf{k}} a_{-\mathbf{k} \tau}^{\dagger} a_{+\mathbf{k} \tau}\right\}, \\
& H_{\lambda}=\sum_{\mathbf{q} \lambda \tau} \varepsilon_{\mathbf{q} \lambda \tau} c_{\mathbf{q} \lambda \tau}^{\dagger} c_{\mathbf{q} \lambda \tau}, \quad \lambda=L, R \\
& H_{T}=\frac{1}{\sqrt{N}} \sum_{\mathbf{k} \mathbf{q} \lambda \tau}\left[T_{\mathbf{k} \lambda \mathbf{q}} c_{\mathbf{q} \lambda \tau}^{\dagger} a_{s \mathbf{k} \tau}+\text { H.c. }\right] .
\end{aligned}
$$

With the unitary transformation

$$
U=\exp \left[-i \frac{\omega_{0} t}{2} \sum_{\mathbf{k} \tau}\left(a_{+\mathbf{k} \tau}^{\dagger} a_{+\mathbf{k} \tau}-a_{-\mathbf{k} \tau}^{\dagger} a_{-\mathbf{k} \tau}\right)\right]
$$


Laser-assisted spin-polarized transport in graphene tunnel junctions

one finds

$$
\begin{aligned}
U^{-1} H_{I} U= & -e v_{F} \exp \left[i \frac{\omega_{0} t}{2} \sum_{\mathbf{k} \tau}\left(a_{+\mathbf{k} \tau}^{\dagger} a_{+\mathbf{k} \tau}-a_{-\mathbf{k} \tau}^{\dagger} a_{-\mathbf{k} \tau}\right)\right] \\
& \times \sum_{\mathbf{k} \tau}\left\{A_{0}^{*} e^{-i \omega_{0} t} d_{+-, \mathbf{k}}^{y} a_{+\mathbf{k} \tau}^{\dagger} a_{-\mathbf{k} \tau}+A_{0} e^{i \omega_{0} t} d_{-+, \mathbf{k}} a_{-\mathbf{k} \tau}^{\dagger} a_{+\mathbf{k} \tau}\right\} \\
& \times \exp \left[-i \frac{\omega_{0} t}{2} \sum_{\mathbf{k} \tau}\left(a_{+\mathbf{k} \tau}^{\dagger} a_{+\mathbf{k} \tau}-a_{-\mathbf{k} \tau}^{\dagger} a_{-\mathbf{k} \tau}\right)\right] \\
= & -e v_{F} \exp \left[i \frac{\omega_{0} t}{2} \sum_{\mathbf{k} \tau}\left(a_{+\mathbf{k} \tau}^{\dagger} a_{+\mathbf{k} \tau}-a_{-\mathbf{k} \tau}^{\dagger} a_{-\mathbf{k} \tau}\right)\right] \sum_{\mathbf{k} \tau} A_{0}^{*} e^{-i \omega_{0} t} d_{+-, \mathbf{k}} a_{+\mathbf{k} \tau}^{\dagger} a_{-\mathbf{k} \tau} \\
& \times \exp \left[-i \frac{\omega_{0} t}{2} \sum_{\mathbf{k} \tau}\left(a_{+\mathbf{k} \tau}^{\dagger} a_{+\mathbf{k} \tau}-a_{-\mathbf{k} \tau}^{\dagger} a_{-\mathbf{k} \tau}\right)\right] \\
& +e v_{F} \exp \left[i \frac{\omega_{0} t}{2} \sum_{\mathbf{k} \tau}\left(a_{+\mathbf{k} \tau}^{\dagger} a_{+\mathbf{k} \tau}-a_{-\mathbf{k} \tau}^{\dagger} a_{-\mathbf{k} \tau}\right)\right] \sum_{\mathbf{k} \tau} A_{0} e^{i \omega_{0} t} d_{-+, \mathbf{k}} a_{-\mathbf{k} \tau}^{\dagger} a_{+\mathbf{k} \tau} \\
& \times \exp \left[-i \frac{\omega_{0} t}{2} \sum_{\mathbf{k} \tau}\left(a_{+\mathbf{k} \tau}^{\dagger} a_{+\mathbf{k} \tau}-a_{-\mathbf{k} \tau}^{\dagger} a_{-\mathbf{k} \tau}\right)\right]
\end{aligned}
$$

The following applies

$$
\begin{aligned}
{\left[i \frac{\omega_{0} t}{2} \sum_{\mathbf{k} \tau}\left(a_{+\mathbf{k} \tau}^{\dagger} a_{+\mathbf{k} \tau}-a_{-\mathbf{k} \tau}^{\dagger} a_{-\mathbf{k} \tau}\right), \sum_{\mathbf{k} \tau} A_{0}^{*} e^{-i \omega_{0} t} d_{+-, \mathbf{k}} a_{+\mathbf{k} \tau}^{\dagger} a_{-\mathbf{k} \tau}\right] } \\
=\sum_{\mathbf{k} \tau \mathbf{k}^{\prime} \tau^{\prime}} \frac{i \omega_{0} t}{2} A_{0}^{*} e^{-i \omega_{0} t} d_{+-, \mathbf{k}^{\prime}}\left[a_{+\mathbf{k} \tau}^{\dagger} a_{+\mathbf{k} \tau}-a_{-\mathbf{k} \tau}^{\dagger} a_{-\mathbf{k} \tau}, a_{+\mathbf{k}^{\prime} \tau^{\prime}}^{\dagger} a_{-\mathbf{k}^{\prime} \tau^{\prime}}\right] \\
=\sum_{\mathbf{k} \tau \mathbf{k}^{\prime} \tau^{\prime}} \frac{i \omega_{0} t}{2} A_{0}^{*} e^{-i \omega_{0} t} d_{+-, \mathbf{k}^{\prime}}\left\{\left[a_{+\mathbf{k} \tau}^{\dagger} a_{+\mathbf{k} \tau}, a_{+\mathbf{k}^{\prime} \tau^{\prime}}^{\dagger} a_{-\mathbf{k}^{\prime} \tau^{\prime}}\right]-\left[a_{-\mathbf{k} \tau}^{\dagger} a_{-\mathbf{k} \tau}, a_{+\mathbf{k}^{\prime} \tau^{\prime}}^{\dagger} a_{-\mathbf{k}^{\prime} \tau^{\prime}}\right]\right\} \\
=\sum_{\mathbf{k} \tau \mathbf{k}^{\prime} \tau^{\prime}} \frac{i \omega_{0} t}{2} A_{0}^{*} e^{-i \omega_{0} t} d_{+-, \mathbf{k}^{\prime}}\left\{a_{+\mathbf{k} \tau}^{\dagger}\left[a_{+\mathbf{k} \tau}, a_{+\mathbf{k}^{\prime} \tau^{\prime}}^{\dagger} a_{-\mathbf{k}^{\prime} \tau^{\prime}}\right]+\left[a_{+\mathbf{k} \tau}^{\dagger}, a_{+\mathbf{k}^{\prime} \tau^{\prime}}^{\dagger} a_{-\mathbf{k}^{\prime} \tau^{\prime}}\right] a_{+\mathbf{k} \tau}\right. \\
\left.=\sum_{-\mathbf{k} \tau}\left[a_{-\mathbf{k} \tau}, a_{+\mathbf{k}^{\prime} \tau^{\prime}}^{\dagger} a_{-\mathbf{k}^{\prime} \tau^{\prime}}\right]-\left[a_{-\mathbf{k} \tau}^{\dagger}, a_{+\mathbf{k}^{\prime} \tau^{\prime}}^{\dagger} a_{-\mathbf{k}^{\prime} \tau^{\prime}}\right] a_{-\mathbf{k} \tau}\right\} \\
=\sum_{\mathbf{k} \tau \mathbf{k}^{\prime} \tau^{\prime}} \frac{i \omega_{0} t}{2} A_{0}^{*} e^{-i \omega_{0} t} d_{+-, \mathbf{k}^{\prime}}\left\{\delta_{\mathbf{k} \mathbf{k}^{\prime}} \delta_{\tau \tau^{\prime}} a_{+\mathbf{k} \tau}^{\dagger} a_{-\mathbf{k}^{\prime} \tau^{\prime}}+\delta_{\mathbf{k} \mathbf{k}^{\prime}} \delta_{\tau \tau^{\prime}} a_{+\mathbf{k}^{\prime} \tau^{\prime}}^{\dagger} a_{-\mathbf{k} \tau}\right\}
\end{aligned}
$$

Furthermore, one finds

$$
\begin{aligned}
& {\left[i \frac{\omega_{0} t}{2} \sum_{\mathbf{k} \tau}\left(a_{+\mathbf{k} \tau}^{\dagger} a_{+\mathbf{k} \tau}-a_{-\mathbf{k} \tau}^{\dagger} a_{-\mathbf{k} \tau}\right), \sum_{\mathbf{k} \tau} A_{0} e^{i \omega_{0} t} d_{-+, \mathbf{k}} a_{-\mathbf{k} \tau}^{\dagger} a_{+\mathbf{k} \tau}\right]} \\
& =\sum_{\mathbf{k} \tau \mathbf{k}^{\prime} \tau^{\prime}} \frac{i \omega_{0} t}{2} A_{0} e^{i \omega_{0} t} d_{-+, \mathbf{k}^{\prime}}\left[a_{+\mathbf{k} \tau}^{\dagger} a_{+\mathbf{k} \tau}-a_{-\mathbf{k} \tau}^{\dagger} a_{-\mathbf{k} \tau}, a_{-\mathbf{k}^{\prime} \tau^{\prime}}^{\dagger} a_{+\mathbf{k}^{\prime} \tau^{\prime}}\right] \\
& =\sum_{\mathbf{k} \tau \mathbf{k}^{\prime} \tau^{\prime}} \frac{i \omega_{0} t}{2} A_{0} e^{i \omega_{0} t} d_{-+, \mathbf{k}^{\prime}}\left\{\left[a_{+\mathbf{k} \tau}^{\dagger} a_{+\mathbf{k} \tau}, a_{-\mathbf{k}^{\prime} \tau^{\prime}}^{\dagger} a_{+\mathbf{k}^{\prime} \tau^{\prime}}\right]-\left[a_{-\mathbf{k} \tau}^{\dagger} a_{-\mathbf{k} \tau}, a_{-\mathbf{k}^{\prime} \tau^{\prime}}^{\dagger} a_{+\mathbf{k}^{\prime} \tau^{\prime}}\right]\right\} \\
& =\sum_{\mathbf{k} \tau \mathbf{k}^{\prime} \tau^{\prime}} \frac{i \omega_{0} t}{2} A_{0} e^{i \omega_{0} t} d_{-+, \mathbf{k}^{\prime}}\left\{a_{+\mathbf{k} \tau}^{\dagger}\left[a_{+\mathbf{k} \tau}, a_{-\mathbf{k}^{\prime} \tau^{\prime}}^{\dagger} a_{+\mathbf{k}^{\prime} \tau^{\prime}}\right]+\left[a_{+\mathbf{k} \tau}^{\dagger}, a_{-\mathbf{k}^{\prime} \tau^{\prime}}^{\dagger} a_{+\mathbf{k}^{\prime} \tau^{\prime}}\right] a_{+\mathbf{k} \tau}\right. \\
& \left.-a_{-\mathbf{k} \tau}^{\dagger}\left[a_{-\mathbf{k} \tau}, a_{-\mathbf{k}^{\prime} \tau^{\prime}}^{\dagger} a_{+\mathbf{k}^{\prime} \tau^{\prime}}\right]-\left[a_{-\mathbf{k} \tau}^{\dagger}, a_{-\mathbf{k}^{\prime} \tau^{\prime}}^{\dagger} a_{+\mathbf{k}^{\prime} \tau^{\prime}}\right] a_{-\mathbf{k} \tau}\right\} \\
& =\sum_{\mathbf{k} \tau \mathbf{k}^{\prime} \tau^{\prime}} \frac{i \omega_{0} t}{2} A_{0} e^{i \omega_{0} t} d_{-+, \mathbf{k}^{\prime}}\left\{-\delta_{\mathbf{k} \mathbf{k}^{\prime}} \delta_{\tau \tau^{\prime}} a_{-\mathbf{k}^{\prime} \tau^{\prime}}^{\dagger} a_{+\mathbf{k}^{\prime} \tau^{\prime}}-\delta_{\mathbf{k} \mathbf{k}^{\prime}} \delta_{\tau \tau^{\prime}} a_{-\mathbf{k} \tau}^{\dagger} a_{+\mathbf{k}^{\prime} \tau^{\prime}}\right\} \\
& =\sum_{\mathbf{k} \tau}\left(-i \omega_{0} t\right) A_{0} e^{i \omega_{0} t} d_{-+, \mathbf{k}} a_{-\mathbf{k} \tau}^{\dagger} a_{+\mathbf{k} \tau} \text {. }
\end{aligned}
$$

Another straightforward calculation is that

$$
\begin{aligned}
U^{-1} H_{G} U & =\sum_{\mathbf{k} s \tau} \epsilon_{s \mathbf{k}} a_{s \mathbf{k} \tau}^{\dagger} a_{s \mathbf{k} \tau}-e v_{F} \sum_{\mathbf{k} \tau}\left\{A_{0}^{*} d_{+-, \mathbf{k}} a_{+\mathbf{k} \tau}^{\dagger} a_{-\mathbf{k} \tau}+A_{0} d_{-+, \mathbf{k}} a_{-\mathbf{k} \tau}^{\dagger} a_{+\mathbf{k} \tau}\right\} \\
U^{-1} H_{T} U & =\frac{1}{\sqrt{N}} \sum_{\mathbf{k} \mathbf{} \lambda \tau}\left[T_{\mathbf{k} \lambda \mathbf{q}} e^{-i \frac{\omega_{0} t}{2}} c_{\mathbf{q} \lambda \tau}^{\dagger} a_{+\mathbf{k} \tau}+T_{\mathbf{k} \lambda \mathbf{q}} e^{i \frac{\omega_{0} t}{2}} c_{\mathbf{q} \lambda \tau}^{\dagger} a_{-\mathbf{k} \tau}+\text { H.c. }\right] \\
& =\frac{1}{\sqrt{N}} \sum_{\mathbf{k} \mathbf{q} \lambda \tau}\left[T_{\mathbf{k} \lambda s \mathbf{q}}(t) c_{\mathbf{q} \lambda \tau}^{\dagger} a_{s \mathbf{k} \tau}+\text { H.c. }\right]
\end{aligned}
$$


where $T_{\mathbf{k} \lambda s \mathbf{q}}(t)=T_{\mathbf{k} \lambda \mathbf{q}} e^{\frac{-i s \omega_{0} t}{2}}$. The final Hamiltonian is expressed in the rotating reference as

$$
\begin{aligned}
\widetilde{H}= & U^{-1} H U+i \frac{d U^{-1}}{d t} U \\
= & \sum_{\mathbf{k} \tau}\left[\left(\epsilon_{+\mathbf{k}}-\frac{\omega_{0}}{2}\right) a_{+\mathbf{k} \tau}^{\dagger} a_{+\mathbf{k} \tau}+\left(\epsilon_{-\mathbf{k}}+\frac{\omega_{0}}{2}\right) a_{-\mathbf{k} \tau}^{\dagger} a_{-\mathbf{k} \tau}\right] \\
& -e v_{F} \sum_{\mathbf{k} \tau}\left\{A_{0}^{*} d_{+-, \mathbf{k}} a_{+\mathbf{k} \tau}^{\dagger} a_{-\mathbf{k} \tau}+A_{0} d_{-+, \mathbf{k}} a_{-\mathbf{k} \tau}^{\dagger} a_{+\mathbf{k} \tau}\right\} \\
& +\sum_{\mathbf{q} \lambda \tau} \varepsilon_{\mathbf{q} \lambda \tau} c_{\mathbf{q} \lambda \tau}^{\dagger} c_{\mathbf{q} \lambda \tau}+\frac{1}{\sqrt{N}} \sum_{\mathbf{k} \mathbf{q} \lambda s \tau}\left[T_{\mathbf{k} \lambda s \mathbf{q}}(t) c_{\mathbf{q} \lambda \tau}^{\dagger} a_{s \mathbf{k} \tau}+\text { H.c. }\right] .
\end{aligned}
$$

Assuming that $p_{x}>>p_{y}$, then $\phi \approx 0, \cos \phi=1$ and the Hamiltonian is further simplified to

$$
\begin{aligned}
\widetilde{H}= & \widetilde{H}_{G}+\widetilde{H}_{L}+\widetilde{H}_{R}+\widetilde{H}_{T} \\
= & \sum_{\mathbf{k} s \tau} \widetilde{\epsilon}_{s \mathbf{k}} a_{s \mathbf{k} \tau}^{\dagger} a_{s \mathbf{k} \tau}+\Delta \sum_{\mathbf{k} \tau}\left(a_{+\mathbf{k} \tau}^{\dagger} a_{-\mathbf{k} \tau}+a_{-\mathbf{k} \tau}^{\dagger} a_{+\mathbf{k} \tau}\right) \\
& +\sum_{\mathbf{q} \lambda \tau} \varepsilon_{\mathbf{q} \lambda \tau} c_{\mathbf{q} \lambda \tau}^{\dagger} c_{\mathbf{q} \lambda \tau}+\frac{1}{\sqrt{N}} \sum_{\mathbf{k} \mathbf{q} \lambda \tau}\left[T_{\mathbf{k} \lambda s \mathbf{q}}(t) c_{\mathbf{q} \lambda \tau}^{\dagger} a_{s \mathbf{k} \tau}+\text { H.c. }\right],
\end{aligned}
$$

where $\widetilde{\epsilon}_{s \mathbf{k}}=\epsilon_{s \mathbf{k}}-\frac{s \omega_{0}}{2}$ and $\Delta=\frac{e v_{F} E_{0}}{2 \omega_{0}}$. The current can be calculated from the time variation of the occupation number operator of the left electrode.

$I_{L}=e\left\langle\dot{\mathcal{N}}_{L}\right\rangle=\frac{i e}{\hbar}\left\langle\left[\widetilde{H}, \mathcal{N}_{L}\right]\right\rangle=\frac{i e}{\hbar} \sum_{\mathbf{q} \tau}\left\langle\left[H, c_{\mathbf{q} L \tau}^{\dagger} c_{\mathbf{q} L \tau}\right]\right\rangle=\frac{i e}{\hbar} \sum_{\mathbf{q} \tau}\left[\left\langle\left[\widetilde{H}_{T}, c_{\mathbf{q} L \tau}^{\dagger}\right] c_{\mathbf{q} L \tau}\right\rangle+\left\langle c_{\mathbf{q} L \tau}^{\dagger}\left[\widetilde{H}_{T}, c_{\mathbf{q} L \tau}\right]\right\rangle\right]$,

The commutation relation reads

$$
\begin{aligned}
{\left[\widetilde{H}_{T}, c_{\mathbf{q} L \tau}^{\dagger}\right] } & =\frac{1}{\sqrt{N}} \sum_{\mathbf{k} \mathbf{q}^{\prime} s \tau^{\prime}} T_{\mathbf{k} L s \mathbf{q}}^{*}(t)\left[a_{s \mathbf{k} \tau^{\prime}}^{\dagger} c_{\mathbf{q}^{\prime} L \tau^{\prime}}, c_{\mathbf{q} L \tau}^{\dagger}\right] \\
& =\frac{1}{\sqrt{N}} \sum_{\mathbf{k} \mathbf{q}^{\prime} s \tau^{\prime}} T_{\mathbf{k} L s \mathbf{q}^{\prime}}^{*}(t) a_{s \mathbf{k} \tau^{\prime}}^{\dagger}\left\{c_{\mathbf{q}^{\prime} L \tau^{\prime}}, c_{\mathbf{q} L \tau}^{\dagger}\right\}=\frac{1}{\sqrt{N}} \sum_{\mathbf{k} s} T_{\mathbf{k} L s \mathbf{q}}^{*}(t) a_{s \mathbf{k} \tau}^{\dagger} \\
{\left[\widetilde{H}_{T}, c_{\mathbf{q} L \tau}\right]=} & -\frac{1}{\sqrt{N}} \sum_{\mathbf{k} s} T_{\mathbf{k} L s \mathbf{q}}(t) a_{s \mathbf{k} \tau} .
\end{aligned}
$$

Thus

$$
I_{L}=e\langle\dot{\mathcal{N}}\rangle=\frac{i e}{\hbar} \frac{1}{\sqrt{N}} \sum_{\mathbf{k q} s \tau}\left[T_{\mathbf{k} L s \mathbf{q}}^{*}(t)\left\langle a_{s \mathbf{k} \tau}^{\dagger} c_{\mathbf{q} L \tau}\right\rangle-T_{\mathbf{k} L s \mathbf{q}}(t)\left\langle c_{\mathbf{q} L \tau}^{\dagger} a_{s \mathbf{k} \tau}\right\rangle\right] .
$$

Define $G_{\mathbf{k} s, \mathbf{q} L}^{\tau^{\prime} \tau,<}\left(t, t^{\prime}\right)=i\left\langle c_{\mathbf{q} L \tau}^{\dagger}\left(t^{\prime}\right) a_{s \mathbf{k} \tau^{\prime}}(t)\right\rangle$, then

$$
I_{L}=-\frac{2 e}{\hbar} \operatorname{Re} \frac{1}{\sqrt{N}} \sum_{\mathbf{k q} s \tau} T_{\mathbf{k} L s \mathbf{q}}(t) G_{\mathbf{k} s, \mathbf{q} L}^{\tau \tau,<}(t, t)
$$

To obtain $G_{\mathbf{k} s, \mathbf{q} L}^{\tau^{\prime} \tau}\left(t, t^{\prime}\right)=i\left\langle c_{\mathbf{q} L \tau}^{\dagger}\left(t^{\prime}\right) a_{s \mathbf{k} \tau^{\prime}}(t)\right\rangle$, one needs to find the time-ordered Green's function, defined as $G_{\mathbf{k} s, \mathbf{q} L}^{\tau \tau^{\prime}, t}\left(t, t^{\prime}\right)=-i\left\langle T\left\{a_{s \mathbf{k} \tau}(t) c_{\mathbf{q} L \tau^{\prime}}^{\dagger}\left(t^{\prime}\right)\right\}\right\rangle$. Using the equationof-motion method we write

$$
\begin{aligned}
& -i \frac{\partial}{\partial t^{\prime}} G_{\mathbf{k} s, \mathbf{q} L}^{\tau \tau^{\prime}, t}\left(t, t^{\prime}\right) \\
= & -i\left\langle T\left\{a_{s \mathbf{k} \tau}\left(t^{\prime}\right)\left[\widetilde{H}, c_{\mathbf{q} L \tau^{\prime}}^{\dagger}\left(t^{\prime}\right)\right]\right\}\right\rangle \\
= & \varepsilon_{\mathbf{q} L \tau^{\prime}}\left[-i\left\langle T\left\{a_{s \mathbf{k} \tau}(t) c_{\mathbf{q} L \tau^{\prime}}^{\dagger}\left(t^{\prime}\right)\right\}\right\rangle\right]+\frac{1}{\sqrt{N}} \sum_{\mathbf{k}^{\prime} s^{\prime}} T_{\mathbf{k}^{\prime} L s^{\prime} \mathbf{q}}^{*}\left(t^{\prime}\right)\left\{\left[-i\left\langle T\left\{a_{s \mathbf{k} \tau}(t) a_{s^{\prime} \mathbf{k}^{\prime} \tau^{\prime}}^{\dagger}\left(t^{\prime}\right)\right\}\right\rangle\right]\right\} \\
= & \varepsilon_{\mathbf{q} L \tau^{\prime}} G_{\mathbf{k} s, \mathbf{q} L}^{\tau \tau^{\prime}, t}\left(t, t^{\prime}\right)+\frac{1}{\sqrt{N}} \sum_{\mathbf{k}^{\prime} s^{\prime}} T_{\mathbf{k}^{\prime} L s^{\prime} \mathbf{q}}^{*}\left(t^{\prime}\right) G_{\mathbf{k} s, \mathbf{k}^{\prime} s^{\prime}}^{\tau \tau^{\prime}}\left(t, t^{\prime}\right),
\end{aligned}
$$


where $G_{\mathbf{k} s, \mathbf{k}^{\prime} s^{\prime}}^{\tau \tau^{\prime}, t}\left(t, t^{\prime}\right)=-i\left\langle T\left\{a_{s \mathbf{k} \tau}(t) a_{s^{\prime} \mathbf{k}^{\prime} \tau^{\prime}}^{\dagger}\left(t^{\prime}\right)\right\}\right\rangle$. Eq.(C.17) can be expressed as

$$
G_{\mathbf{k} s, \mathbf{q} L}^{\tau \tau^{\prime}, t}\left(t, t^{\prime}\right)=\frac{1}{\sqrt{N}} \sum_{\mathbf{k}^{\prime} s^{\prime}} \int d t_{1} G_{\mathbf{k} s, \mathbf{k}^{\prime} s^{\prime}}^{\tau \tau^{\prime}, t}\left(t, t_{1}\right) T_{\mathbf{k}^{\prime} L s^{\prime} \mathbf{q}}^{*}\left(t_{1}\right) g_{\mathbf{q} L}^{\tau^{\prime} \tau^{\prime}, t}\left(t_{1}-t^{\prime}\right)
$$

Make the analytic continuation to Eq.(C.18),

$G_{\mathbf{k} s, \mathbf{q} L}^{\tau \tau^{\prime},<}\left(t, t^{\prime}\right)=\frac{1}{\sqrt{N}} \sum_{\mathbf{k}^{\prime} s^{\prime}} \int d t_{1} T_{\mathbf{k}^{\prime} L s^{\prime} \mathbf{q}}^{*}\left(t_{1}\right)\left[G_{\mathbf{k} s, \mathbf{k}^{\prime} s^{\prime}}^{\tau \tau^{\prime}, r}\left(t, t_{1}\right) g_{\mathbf{q} L}^{\tau^{\prime} \tau^{\prime},<}\left(t_{1}-t^{\prime}\right)+G_{\mathbf{k} s, \mathbf{k}^{\prime} s^{\prime}}^{\tau \tau^{\prime}}\left(t, t_{1}\right) g_{\mathbf{q} L}^{\tau^{\prime} \tau^{\prime}, a}\left(t_{1}-t^{\prime}\right)\right]$

Substitute Eq.(C.19) in Eq.(C.16),

$$
\begin{aligned}
I_{L}= & -\frac{2 e}{\hbar} \operatorname{Re} \frac{1}{N} \sum_{\mathbf{k q} s \tau} \sum_{\mathbf{k}^{\prime} s^{\prime}} \int d t_{1} T_{\mathbf{k} L s \mathbf{q}}(t) T_{\mathbf{k}^{\prime} L s^{\prime} \mathbf{q}}^{*}\left(t_{1}\right)\left[G_{\mathbf{k} s, \mathbf{k}^{\prime} s^{\prime}}^{\tau \tau, r}\left(t, t_{1}\right) g_{\mathbf{q} L}^{\tau \tau,<}\left(t_{1}-t\right)\right. \\
+ & \left.G_{\mathbf{k} s, \mathbf{k}^{\prime} s^{\prime}}^{\tau \tau,<}\left(t, t_{1}\right) g_{\mathbf{q} L}^{\tau \tau, a}\left(t_{1}-t\right)\right] \\
= & -\frac{2 e}{\hbar} \operatorname{Re} \frac{1}{N} \sum_{\mathbf{k q} s \tau} \sum_{\mathbf{k}^{\prime} s^{\prime}} \int \frac{d \varepsilon}{2 \pi} \int d t_{1} e^{-i \varepsilon\left(t_{1}-t\right)} T_{\mathbf{k} L s \mathbf{q}}(t) T_{\mathbf{k}^{\prime} L s^{\prime} \mathbf{q}}^{*}\left(t_{1}\right)\left[G_{\mathbf{k} s, \mathbf{k}^{\prime} s^{\prime}}^{\tau \tau, r}\left(t, t_{1}\right) g_{\mathbf{q} L}^{\tau \tau,<}(\varepsilon)\right. \\
+ & \left.G_{\mathbf{k} s, \mathbf{k}^{\prime} s^{\prime}}^{\tau \tau,<}\left(t, t_{1}\right) g_{\mathbf{q} L}^{\tau \tau, a}(\varepsilon)\right] \\
= & -\frac{2 e}{\hbar} \operatorname{Re} \frac{1}{N} \sum_{\mathbf{k q} s \tau \mathbf{k}^{\prime} s^{\prime}} \int \frac{d \varepsilon}{2 \pi} \int d t_{1} e^{-i \varepsilon\left(t_{1}-t\right)} T_{\mathbf{k} L \mathbf{q}} T_{\mathbf{k}^{\prime} L \mathbf{q}}^{*} e^{-\frac{i s \omega_{0} t}{2}} e^{\frac{i s^{\prime} \omega_{0} t_{1}}{2}} \\
& \times\left[G_{\mathbf{k} s, \mathbf{k}^{\prime} s^{\prime}}^{\tau \tau, r}\left(t, t_{1}\right) g_{\mathbf{q} L}^{\tau \tau,<}(\varepsilon)+G_{\mathbf{k} s, \mathbf{k}^{\prime} s^{\prime}}^{\tau \tau,<}\left(t, t_{1}\right) g_{\mathbf{q} L}^{\tau \tau, a}(\varepsilon)\right]
\end{aligned}
$$

where $g_{\mathbf{q} \lambda}^{\tau \tau, r, a}(\varepsilon)=\frac{1}{\varepsilon-\varepsilon_{\mathbf{q} \lambda \tau} \pm i \eta}$ and $g_{\mathbf{q} \lambda}^{\tau \tau,<}(\varepsilon)=i 2 \pi f_{\lambda}\left(\varepsilon_{\mathbf{q} \lambda \tau}\right) \delta\left(\varepsilon-\varepsilon_{\mathbf{q} \lambda \tau}\right)$ with $f_{\lambda}\left(\varepsilon_{\mathbf{q} \lambda \tau}\right)$ denoting the Fermi distribution function in the $\lambda$ ferromagnetic electrode. Let $\Gamma_{L \mathbf{k k}^{\prime}}^{\tau}(\varepsilon)=$ $2 \pi \sum_{\mathbf{q}} T_{\mathbf{k} L \mathbf{q}}^{*} T_{\mathbf{k}^{\prime} L \mathbf{q}} \delta\left(\varepsilon-\varepsilon_{\mathbf{q} L \tau}\right)$, then Eq.(C.20) can be further expressed as

$$
\begin{aligned}
I_{L}= & -\frac{e}{\hbar} R e \frac{1}{N} \sum_{\mathbf{k} s \tau} \sum_{\mathbf{k}^{\prime} s^{\prime}} \int \frac{d \varepsilon}{2 \pi} \int d t_{1} e^{-i \varepsilon\left(t_{1}-t\right)} e^{-\frac{i s \omega_{0} t}{2}} e^{\frac{i s^{\prime} \omega_{0} t_{1}}{2}} \\
& \times\left\{2 G_{\mathbf{k} s, \mathbf{k}^{\prime} s^{\prime}}^{\tau \tau}\left(t, t_{1}\right) f_{L}(\varepsilon) i \Gamma_{L, \mathbf{k}^{\prime} \mathbf{k}}^{\tau}+G_{\mathbf{k} s, \mathbf{k}^{\prime} s^{\prime}}^{\tau \tau,<}\left(t, t_{1}\right) i \Gamma_{L, \mathbf{k}^{\prime} \mathbf{k}}^{\tau}\right\}
\end{aligned}
$$

\section{Appendix D. Derivation of Green's functions}

Appendix D.1. The Green's function for the central region coupled to the electrodes

We note the following commutation relations

$$
\begin{aligned}
{\left[a_{s \mathbf{k} \tau}, \widetilde{H}\right]=} & {\left[a_{s \mathbf{k} \tau}, \widetilde{H}_{G}\right]+\left[a_{s \mathbf{k} \tau}, \widetilde{H}_{T}\right]=\widetilde{\epsilon}_{s \mathbf{k}} a_{s \mathbf{k} \tau}+\delta_{s+} \Delta a_{-\mathbf{k} \tau}+\delta_{s-} \Delta a_{+\mathbf{k} \tau} } \\
& +\frac{1}{\sqrt{N}} \sum_{\mathbf{q} \lambda} T_{\mathbf{k} \lambda s \mathbf{q}}^{*}(t) c_{\mathbf{q} \lambda \tau}
\end{aligned}
$$

with $\widetilde{\epsilon}_{s \mathbf{k}}=\epsilon_{s \mathbf{k}}-s \omega_{0} / 2=s v_{F}|\mathbf{k}|-s \omega_{0} / 2$. The retarded Green's function $G_{\mathbf{k} s, \mathbf{k}^{\prime} s^{\prime}}^{\tau \tau^{\prime}, r}\left(t, t^{\prime}\right)=$ $-i \theta\left(t-t^{\prime}\right)\left\langle\left\{a_{s \mathbf{k} \tau}(t), a_{s^{\prime} \mathbf{k}^{\prime} \tau^{\prime}}^{\dagger}\left(t^{\prime}\right)\right\}\right\rangle$ satisfies

$$
\begin{aligned}
i \frac{\partial}{\partial t} G_{\mathbf{k} s, \mathbf{k}^{\prime} s^{\prime}}^{\tau \tau^{\prime}, r} & \left(t, t^{\prime}\right)=\delta\left(t-t^{\prime}\right) \delta_{\mathbf{k k}^{\prime}} \delta_{s s^{\prime}} \delta_{\tau \tau^{\prime}}-i \theta\left(t-t^{\prime}\right)\left\langle\left\{\left[a_{s \mathbf{k} \tau}(t), \widetilde{H}\right], a_{s^{\prime} \mathbf{k}^{\prime} \tau^{\prime}}^{\dagger}\left(t^{\prime}\right)\right\}\right\rangle \\
= & \delta\left(t-t^{\prime}\right) \delta_{\mathbf{k k}^{\prime}} \delta_{s s^{\prime}} \delta_{\tau \tau^{\prime}}+\widetilde{\epsilon}_{s \mathbf{k}} G_{\mathbf{k} s, \mathbf{k}^{\prime} s^{\prime}}^{\tau \tau^{\prime},}\left(t, t^{\prime}\right)+\delta_{s+} \Delta G_{\mathbf{k}-, \mathbf{k}^{\prime} s^{\prime}}^{\tau \tau^{\prime}, r}\left(t, t^{\prime}\right)+\delta_{s-} \Delta G_{\mathbf{k}+, \mathbf{k}^{\prime} s^{\prime}}^{\tau \tau^{\prime}, r}\left(t, t^{\prime}\right) \\
& +\frac{1}{\sqrt{N}} \sum_{\mathbf{q} \lambda} T_{\mathbf{k} \lambda s \mathbf{q}}^{*}(t) G_{\mathbf{q} \lambda, \mathbf{k}^{\prime} s^{\prime}}^{\tau \tau^{\prime}, r}\left(t, t^{\prime}\right) .
\end{aligned}
$$

In addition,

$$
i \frac{\partial}{\partial t} G_{\mathbf{q} \lambda, \mathbf{k}^{\prime} s^{\prime}}^{\tau \tau^{\prime}, r}\left(t, t^{\prime}\right)=\varepsilon_{\mathbf{q} \lambda \tau} G_{\mathbf{q} \lambda, \mathbf{k}^{\prime} s^{\prime}}^{\tau \tau^{\prime}, r}\left(t, t^{\prime}\right)+\frac{1}{\sqrt{N}} \sum_{\mathbf{k}_{1} s_{1}} T_{\mathbf{k}_{1} \lambda s_{1} \mathbf{q}}(t) G_{\mathbf{k}_{1} s_{1}, \mathbf{k}^{\prime} s^{\prime}}^{\tau \tau^{\prime}, r}\left(t, t^{\prime}\right)
$$


or

$$
G_{\mathbf{q} \lambda, \mathbf{k}^{\prime} s^{\prime}}^{\tau \tau^{\prime}, r}\left(t, t^{\prime}\right)=\frac{1}{\sqrt{N}} \sum_{\mathbf{k}_{1} s_{1}} \int d t_{1} g_{\mathbf{q} \lambda}^{\tau \tau, r}\left(t-t_{1}\right) T_{\mathbf{k}_{1} \lambda s_{1} \mathbf{q}}\left(t_{1}\right) G_{\mathbf{k}_{1} s_{1}, \mathbf{k}^{\prime} s^{\prime}}^{\tau \tau^{\prime}, r}\left(t_{1}, t^{\prime}\right) .
$$

Substituting Eq.(D.5) into Eq.(D.3) leads to

$$
\begin{aligned}
& i \frac{\partial}{\partial t} G_{\mathbf{k} s, \mathbf{k}^{\prime} s^{\prime}}^{\tau \tau^{\prime}, r}\left(t, t^{\prime}\right) \\
& =\delta\left(t-t^{\prime}\right) \delta_{\mathbf{k k}^{\prime}} \delta_{s s^{\prime}} \delta_{\tau \tau^{\prime}}+\widetilde{\epsilon}_{s \mathbf{k}} G_{\mathbf{k} s, \mathbf{k}^{\prime} s^{\prime}}^{\tau \tau^{\prime}, r}\left(t, t^{\prime}\right)+\delta_{s+} \Delta G_{\mathbf{k}-, \mathbf{k}^{\prime} s^{\prime}}^{\tau \tau^{\prime}, r}\left(t, t^{\prime}\right)+\delta_{s-} \Delta G_{\mathbf{k}+, \mathbf{k}^{\prime} s^{\prime}}^{\tau \tau^{\prime}, r}\left(t, t^{\prime}\right) \\
& +\frac{1}{N} \sum_{\mathbf{q} \lambda} \sum_{\mathbf{k}_{1} s_{1}} \int d t_{1} T_{\mathbf{k} \lambda s \mathbf{q}}^{*}(t) g_{\mathbf{q} \lambda}^{\tau \tau, r}\left(t-t_{1}\right) T_{\mathbf{k}_{1} \lambda s_{1} \mathbf{q}}\left(t_{1}\right) G_{\mathbf{k}_{1} s_{1}, \mathbf{k}^{\prime} s^{\prime}}^{\tau \tau^{\prime}, r}\left(t_{1}, t^{\prime}\right) \\
& =\delta\left(t-t^{\prime}\right) \delta_{\mathbf{k k}^{\prime}} \delta_{s s^{\prime}} \delta_{\tau \tau^{\prime}}+\widetilde{\epsilon}_{s \mathbf{k}} G_{\mathbf{k} s, \mathbf{k}^{\prime} s^{\prime}}^{\tau \tau^{\prime}, r}\left(t, t^{\prime}\right)+\delta_{s+} \Delta G_{\mathbf{k}-, \mathbf{k}^{\prime} s^{\prime}}^{\tau \tau^{\prime}, r}\left(t, t^{\prime}\right)+\delta_{s-} \Delta G_{\mathbf{k}+, \mathbf{k}^{\prime} s^{\prime}}^{\tau \tau^{\prime}, r}\left(t, t^{\prime}\right) \\
& +\frac{1}{N} \sum_{\mathbf{q} \lambda} \sum_{\mathbf{k}_{1} s_{1}} \int \frac{d \varepsilon}{2 \pi} \int d t_{1} e^{-i \varepsilon\left(t-t_{1}\right)} T_{\mathbf{k} \lambda \mathbf{q}}^{*} g_{\mathbf{q} \lambda}^{\tau \tau, r}(\varepsilon) T_{\mathbf{k}_{1} \lambda \mathbf{q}} e^{\frac{i s \omega_{0} t}{2}} e^{-\frac{i s_{1} \omega_{0} t_{1}}{2}} G_{\mathbf{k}_{1} s_{1}, \mathbf{k}^{\prime} s^{\prime}}^{\tau \tau^{\prime}, r}\left(t_{1}, t^{\prime}\right) \\
& =\delta\left(t-t^{\prime}\right) \delta_{\mathbf{k k}^{\prime}} \delta_{s s^{\prime}} \delta_{\tau \tau^{\prime}}+\widetilde{\epsilon}_{s \mathbf{k}} G_{\mathbf{k} s, \mathbf{k}^{\prime} s^{\prime}}^{\tau \tau^{\prime}, r}\left(t, t^{\prime}\right)+\delta_{s+} \Delta G_{\mathbf{k}-, \mathbf{k}^{\prime} s^{\prime}}^{\tau \tau^{\prime}, r}\left(t, t^{\prime}\right)+\delta_{s-} \Delta G_{\mathbf{k}+, \mathbf{k}^{\prime} s^{\prime}}^{\tau \tau^{\prime}}\left(t, t^{\prime}\right) \\
& \left.+\frac{1}{N} \sum_{\lambda \mathbf{k}_{1} s_{1}} \int \frac{d \varepsilon}{2 \pi} \int d t_{1} e^{-i \varepsilon\left(t-t_{1}\right)} e^{\frac{i s \omega_{0} t}{2}} e^{-\frac{i s_{1} \omega_{0} t_{1}}{2}} \frac{-i}{2} \Gamma_{\lambda, \mathbf{k k}_{1}}^{\tau} G_{\mathbf{k}_{1} s_{1}, \mathbf{k}^{\prime} s^{\prime}}^{\tau \tau^{\prime}, t_{1}}, t^{\prime}\right) .
\end{aligned}
$$

The above expression can be written as

$$
G_{\mathbf{k}, \mathbf{k}^{\prime}}^{\tau \tau^{\prime}, r}\left(t, t^{\prime}\right)=\delta_{\mathbf{k k}^{\prime}} \delta_{\tau \tau^{\prime}} g_{\mathbf{k}}^{\tau \tau, r}\left(t-t^{\prime}\right)+\frac{1}{N} \sum_{\mathbf{k}_{1}} \int d t_{1} d t_{2} g_{\mathbf{k}}^{\tau \tau, r}\left(t-t_{1}\right) \Sigma^{\tau, r}\left(t_{1}, t_{2}\right) G_{\mathbf{k}_{1}, \mathbf{k}^{\prime}}^{\tau \tau^{\prime}, r}\left(t_{2}, t^{\prime}\right)
$$

where the quantities without the $s$ and $s^{\prime}$ indexes means matrixes in $s, s^{\prime}$ space implicitly, and

$$
\begin{aligned}
g_{\mathbf{k}}^{\tau \tau, r}(t) & =\left(\begin{array}{cc}
i \frac{\partial}{\partial t}-\widetilde{\epsilon}_{+\mathbf{k}} & -\Delta \\
-\Delta & i \frac{\partial}{\partial t}-\widetilde{\epsilon}_{-\mathbf{k}}
\end{array}\right)^{-1} \\
& =\int \frac{d \varepsilon}{2 \pi} e^{-i \varepsilon t}\left(\begin{array}{cc}
\frac{\varepsilon-\widetilde{\epsilon}_{-\mathbf{k}}}{\left(\varepsilon-\widetilde{\epsilon}_{+\mathbf{k}}\right)\left(\varepsilon-\widetilde{\epsilon}_{-\mathbf{k}}\right)-\Delta^{2}} & \frac{\Delta}{\left(\varepsilon-\widetilde{\epsilon}_{+\mathbf{k}}\right)\left(\varepsilon-\widetilde{\epsilon}_{-\mathbf{k}}\right)-\Delta^{2}} \\
\frac{\left.\varepsilon-\widetilde{\epsilon}_{+\mathbf{k}}\right)\left(\varepsilon-\widetilde{\epsilon}_{-\mathbf{k}}\right)-\Delta^{2}}{\left(\varepsilon-\widetilde{\epsilon}_{+\mathbf{k}}\right)\left(\varepsilon-\widetilde{\epsilon}_{-\mathbf{k}}\right)-\Delta^{2}}
\end{array}\right)
\end{aligned}
$$

is the Green's function for graphene without the coupling between the electrodes and the graphene. The selfenergy reads

$$
\Sigma^{\tau, r}\left(t, t^{\prime}\right)=\int \frac{d \varepsilon}{2 \pi} \Sigma^{\tau, r} e^{-i \varepsilon\left(t-t^{\prime}\right)}\left(\begin{array}{cc}
e^{i \frac{\omega_{0}}{2}\left(t-t^{\prime}\right)} & e^{i \frac{\omega_{0}}{2}\left(t+t^{\prime}\right)} \\
e^{-i \frac{\omega_{0}}{2}\left(t+t^{\prime}\right)} & e^{-i \frac{\omega_{0}}{2}\left(t-t^{\prime}\right)}
\end{array}\right)
$$

with $\Sigma^{\tau, r}=-\frac{i}{2}\left(\Gamma_{L}^{\tau}+\Gamma_{R}^{\tau}\right)$. Let $\mathcal{G}^{\tau \tau^{\prime}, r}\left(t, t^{\prime}\right)=\frac{1}{N} \sum_{\mathbf{k k}^{\prime}} G_{\mathbf{k}, \mathbf{k}^{\prime}}^{\tau \tau^{\prime}, r}\left(t, t^{\prime}\right), g^{\tau \tau^{\prime}, r}(t)=\frac{1}{N} \sum_{\mathbf{k}} g_{\mathbf{k}}^{\tau \tau^{\prime}, r}(t)$, Eq.(D.7) leads to

$$
\mathcal{G}^{\tau \tau^{\prime}, r}\left(t, t^{\prime}\right)=\delta_{\tau \tau^{\prime}} g^{\tau \tau, r}\left(t-t^{\prime}\right)+\int d t_{1} \int d t_{2} g^{\tau \tau, r}\left(t-t_{1}\right) \Sigma^{\tau, r}\left(t_{1}, t_{2}\right) \mathcal{G}^{\tau \tau^{\prime}, r}\left(t_{2}, t^{\prime}\right) .
$$

Appendix D.2. The Green's functions for the central region without coupling to the electrodes

Below, we attempt to calculate $g^{\tau \tau, r}(t)$.

$$
\begin{aligned}
& g_{++}^{\tau \tau, r}=\frac{1}{N} \sum_{\mathbf{k}} \frac{\varepsilon-\widetilde{\epsilon}_{-\mathbf{k}}}{\left(\varepsilon-\widetilde{\epsilon}_{+\mathbf{k}}\right)\left(\varepsilon-\widetilde{\epsilon}_{-\mathbf{k}}\right)-\Delta^{2}} \\
& =\frac{S}{(2 \pi)^{2} N} \int k d k d \phi \frac{\varepsilon-V_{g}+\left(v_{F} k-\frac{\omega_{0}}{2}\right)}{\left(\varepsilon-V_{g}\right)^{2}-\left(v_{F} k-\frac{\omega_{0}}{2}\right)^{2}-\Delta^{2}} \\
& =\frac{1}{(2 \pi)^{2} \rho} \frac{2 \pi}{v_{F}^{2}} \int_{-\frac{\omega_{0}}{2}}^{D-\omega_{0} / 2} d \varepsilon_{k} \frac{\left(\varepsilon_{k}+\frac{\omega_{0}}{2}\right)\left[\left(\varepsilon-V_{g}\right)+\varepsilon_{k}\right]}{\left(\varepsilon-V_{g}\right)^{2}-\varepsilon_{k}^{2}-\Delta^{2}+i \operatorname{sgn}\left(\varepsilon-V_{g}\right) \eta} \\
& =\frac{1}{(2 \pi)^{2} \rho} \frac{2 \pi}{v_{F}^{2}} \int_{-\frac{\omega_{0}}{2}}^{D-\omega_{0} / 2} d \varepsilon_{k}\left[\frac{\varepsilon_{k}\left(\varepsilon-V_{g}+\varepsilon_{k}\right)}{\left(\varepsilon-V_{g}\right)^{2}-\varepsilon_{k}^{2}-\Delta^{2}+i \operatorname{sgn}\left(\varepsilon-V_{g}\right) \eta}+\frac{\frac{\omega_{0}}{2}\left(\varepsilon-V_{g}+\varepsilon_{k}\right)}{\left(\varepsilon-V_{g}\right)^{2}-\varepsilon_{k}^{2}-\Delta^{2}+i \operatorname{sgn}\left(\varepsilon-V_{g}\right) \eta}\right] \\
& =-\frac{1}{(2 \pi)^{2} \rho} \frac{2 \pi}{v_{F}^{2}} \int_{-\frac{\omega_{0}}{2}}^{D-\omega_{0} / 2} d \varepsilon_{k}\left[\frac{\varepsilon_{k}\left(\varepsilon-V_{g}+\varepsilon_{k}\right)}{\varepsilon_{k}^{2}-\left(\varepsilon-V_{g}\right)^{2}+\Delta^{2}-i \operatorname{sgn}\left(\varepsilon-V_{g}\right) \eta}+\frac{\frac{\omega_{0}}{2}\left(\varepsilon-V_{g}+\varepsilon_{k}\right)}{\varepsilon_{k}^{2}-\left(\varepsilon-V_{g}\right)^{2}+\Delta^{2}-i \operatorname{sgn}\left(\varepsilon-V_{g}\right) \eta}\right] \text {, }
\end{aligned}
$$


If $\left|\varepsilon-V_{g}\right|<\Delta$, then

$$
\begin{aligned}
& -\frac{1}{2 \pi \rho v_{F}^{2}} \int_{-\frac{\omega_{0}}{2}}^{D-\omega_{0} / 2} d \varepsilon_{k} \frac{\varepsilon_{k}\left(\varepsilon-V_{g}+\varepsilon_{k}\right)}{\varepsilon_{k}^{2}-\left(\varepsilon-V_{g}\right)^{2}+\Delta^{2}} \\
= & -\frac{1}{2 \pi \rho v_{F}^{2}} \int_{-\frac{\omega_{0}}{2}}^{D-\omega_{0} / 2} d \varepsilon_{k}\left[1+\frac{\varepsilon_{k}\left(\varepsilon-V_{g}\right)+\left(\varepsilon-V_{g}\right)^{2}-\Delta^{2}}{\varepsilon_{k}^{2}-\left(\varepsilon-V_{g}\right)^{2}+\Delta^{2}}\right] \\
= & -\frac{1}{2 \pi \rho v_{F}^{2}} \int_{-\frac{\omega_{0}}{2}}^{D-\omega_{0} / 2} d \varepsilon_{k}\left[1+\frac{\varepsilon_{k}\left(\varepsilon-V_{g}\right)}{\varepsilon_{k}^{2}-\left(\varepsilon-V_{g}\right)^{2}+\Delta^{2}}+\frac{\left(\varepsilon-V_{g}\right)^{2}-\Delta^{2}}{\varepsilon_{k}^{2}-\left(\varepsilon-V_{g}\right)^{2}+\Delta^{2}}\right] \\
= & -\frac{1}{2 \pi \rho v_{F}^{2}}\left\{\frac{\left(\varepsilon-V_{g}\right)}{2} \ln \left|\frac{\left(D-\omega_{0} / 2\right)^{2}+\Delta^{2}-\left(\varepsilon-V_{g}\right)^{2}}{\omega_{0}^{2} / 4+\Delta^{2}-\left(\varepsilon-V_{g}\right)^{2}}\right|\right. \\
& \left.-\sqrt{\Delta^{2}-\left(\varepsilon-V_{g}\right)^{2}}\left[\arctan \frac{D-\omega_{0} / 2}{\sqrt{\Delta^{2}-\left(\varepsilon-V_{g}\right)^{2}}}-\arctan \frac{-\omega_{0} / 2}{\sqrt{\Delta^{2}-\left(\varepsilon-V_{g}\right)^{2}}}\right]+D\right\} .
\end{aligned}
$$

From the above calculations, one readily finds that when the gate voltage is taken into account, the Green's functions for the irradiated graphene are obtained only by changing $\varepsilon$ to $\varepsilon-V_{g}$.

$$
\begin{aligned}
& -\frac{1}{2 \pi \rho v_{F}^{2}} \int_{-\frac{\omega_{0}}{2}}^{D-\omega_{0} / 2} d \varepsilon_{k} \frac{\frac{\omega_{0}}{2}\left(\varepsilon+\varepsilon_{k}\right)}{\varepsilon_{k}^{2}-\varepsilon^{2}+\Delta^{2}} \\
= & -\frac{1}{2 \pi \rho v_{F}^{2}} \int_{-\frac{\omega_{0}}{2}}^{D-\omega_{0} / 2} d \varepsilon_{k}\left[\frac{\frac{\omega_{0}}{2} \varepsilon_{k}}{\varepsilon_{k}^{2}-\varepsilon^{2}+\Delta^{2}}+\frac{\frac{\omega_{0}}{2} \varepsilon}{\varepsilon_{k}^{2}-\varepsilon^{2}+\Delta^{2}}\right] \\
= & -\frac{1}{2 \pi \rho v_{F}^{2}}\left\{\frac{\omega_{0}}{4} \ln \left|\frac{\left(D-\omega_{0} / 2\right)^{2}+\Delta^{2}-\varepsilon^{2}}{\omega_{0}^{2} / 4+\Delta^{2}-\varepsilon^{2}}\right|+\frac{\frac{\omega_{0}}{2} \varepsilon}{\sqrt{\Delta^{2}-\varepsilon^{2}}}\left[\arctan \frac{D-\omega_{0} / 2}{\sqrt{\Delta^{2}-\varepsilon^{2}}}-\arctan \frac{-\omega_{0} / 2}{\sqrt{\Delta^{2}-\varepsilon^{2}}}\right]\right\} .
\end{aligned}
$$

Thus

$$
\begin{aligned}
& g_{++}^{\tau \tau, r}=-\frac{1}{2 \pi \rho v_{F}^{2}}\left\{\left(\frac{\varepsilon}{2}+\frac{\omega_{0}}{4}\right) \ln \left|\frac{\left(D-\omega_{0} / 2\right)^{2}+\Delta^{2}-\varepsilon^{2}}{\omega_{0}^{2} / 4+\Delta^{2}-\varepsilon^{2}}\right|\right. \\
& \left.-\frac{1}{\sqrt{\Delta^{2}-\varepsilon^{2}}}\left(\Delta^{2}-\varepsilon^{2}-\frac{\omega_{0}}{2} \varepsilon\right)\left[\arctan \frac{D-\omega_{0} / 2}{\sqrt{\Delta^{2}-\varepsilon^{2}}}-\arctan \frac{-\omega_{0} / 2}{\sqrt{\Delta^{2}-\varepsilon^{2}}}\right]+D\right\} \text {, } \\
& g_{--}^{\tau \tau, r}=-\frac{1}{2 \pi \rho v_{F}^{2}} \int_{-\frac{\omega_{0}}{2}}^{D-\omega_{0} / 2} d \varepsilon_{k} \frac{\left(\varepsilon_{k}+\frac{\omega_{0}}{2}\right)\left(\varepsilon-\varepsilon_{k}\right)}{\varepsilon_{k}^{2}-\varepsilon^{2}+\Delta^{2}}=-\frac{1}{2 \pi \rho v_{F}^{2}} \int_{-\frac{\omega_{0}}{2}}^{D-\omega_{0} / 2} d \varepsilon_{k}\left[\frac{\varepsilon_{k}\left(\varepsilon-\varepsilon_{k}\right)}{\varepsilon_{k}^{2}-\varepsilon^{2}+\Delta^{2}}+\frac{\frac{\omega_{0}}{2}\left(\varepsilon-\varepsilon_{k}\right)}{\varepsilon_{k}^{2}-\varepsilon^{2}+\Delta^{2}}\right] \\
& =-\frac{1}{2 \pi \rho v_{F}^{2}} \int_{-\frac{\omega_{0}}{2}}^{D-\omega_{0} / 2} d \varepsilon_{k}\left[\frac{\varepsilon_{k} \varepsilon}{\varepsilon_{k}^{2}+\Delta^{2}-\varepsilon^{2}}+\frac{\Delta^{2}-\varepsilon^{2}}{\varepsilon_{k}^{2}+\Delta^{2}-\varepsilon^{2}}-1+\frac{\frac{\omega_{0}}{2}\left(\varepsilon-\varepsilon_{k}\right)}{\varepsilon_{k}^{2}-\varepsilon^{2}+\Delta^{2}}\right] \\
& =-\frac{1}{2 \pi \rho v_{F}^{2}} \int_{-\frac{\omega_{0}}{2}}^{D-\omega_{0} / 2} d \varepsilon_{k}\left[\frac{\varepsilon_{k}\left(\varepsilon-\omega_{0} / 2\right)}{\varepsilon_{k}^{2}+\Delta^{2}-\varepsilon^{2}}+\frac{\Delta^{2}-\varepsilon^{2}+\varepsilon \omega_{0} / 2}{\varepsilon_{k}^{2}+\Delta^{2}-\varepsilon^{2}}-1\right] \\
& =-\frac{1}{2 \pi \rho v_{F}^{2}}\left\{\left(\frac{\varepsilon}{2}-\frac{\omega_{0}}{4}\right) \ln \left|\frac{\left(D-\omega_{0} / 2\right)^{2}+\Delta^{2}-\varepsilon^{2}}{\omega_{0}^{2} / 4+\Delta^{2}-\varepsilon^{2}}\right|+\frac{\Delta^{2}-\varepsilon^{2}+\varepsilon \omega_{0} / 2}{\sqrt{\Delta^{2}-\varepsilon^{2}}}\left[\arctan \frac{D-\omega_{0} / 2}{\sqrt{\Delta^{2}-\varepsilon^{2}}}-\arctan \frac{-\omega_{0} / 2}{\sqrt{\Delta^{2}-\varepsilon^{2}}}\right]\right. \\
& -D\} \\
& g_{-+}^{\tau \tau, r}=g_{+-}^{\tau \tau, r}=-\frac{1}{2 \pi \rho v_{F}^{2}} \int_{-\frac{\omega_{0}}{2}}^{D-\omega_{0} / 2} d \varepsilon_{k} \frac{\left(\varepsilon_{k}+\omega_{0} / 2\right) \Delta}{\varepsilon_{k}^{2}-\varepsilon^{2}+\Delta^{2}}=-\frac{1}{2 \pi \rho v_{F}^{2}} \int_{-\frac{\omega_{0}}{2}}^{D-\omega_{0} / 2} d \varepsilon_{k}\left[\frac{\varepsilon_{k} \Delta}{\varepsilon_{k}^{2}-\varepsilon^{2}+\Delta^{2}}+\frac{\Delta \omega_{0} / 2}{\varepsilon_{k}^{2}-\varepsilon^{2}+\Delta^{2}}\right] \\
& =-\frac{1}{2 \pi \rho v_{F}^{2}}\left\{\frac{\Delta}{2} \ln \left|\frac{\left(D-\omega_{0} / 2\right)^{2}+\Delta^{2}-\varepsilon^{2}}{\omega_{0}^{2} / 4+\Delta^{2}-\varepsilon^{2}}\right|+\frac{\Delta \omega_{0} / 2}{\sqrt{\Delta^{2}-\varepsilon^{2}}}\left[\arctan \frac{D-\omega_{0} / 2}{\sqrt{\Delta^{2}-\varepsilon^{2}}}-\arctan \frac{-\omega_{0} / 2}{\sqrt{\Delta^{2}-\varepsilon^{2}}}\right]\right\} \text {. }
\end{aligned}
$$

For $|\varepsilon|>\Delta$, we have

$$
\begin{aligned}
g_{++}^{\tau \tau, r} & =\frac{1}{N} \sum_{\mathbf{k}} \frac{\varepsilon-\widetilde{\epsilon}_{-\mathbf{k}}}{\left(\varepsilon-\widetilde{\epsilon}_{+\mathbf{k}}\right)\left(\varepsilon-\widetilde{\epsilon}_{-\mathbf{k}}\right)-\Delta^{2}} \\
& =\frac{S}{(2 \pi)^{2} N} \int k d k d \phi \frac{\varepsilon+\left(v_{F} k-\frac{\omega_{0}}{2}\right)}{\varepsilon^{2}-\left(v_{F} k-\frac{\omega_{0}}{2}\right)^{2}-\Delta^{2}} \\
& =\frac{1}{(2 \pi)^{2} \rho} \frac{2 \pi}{v_{F}^{2}} \int_{-\frac{\omega_{0}}{2}}^{D-\omega_{0} / 2}\left(\varepsilon_{k}+\frac{\omega_{0}}{2}\right) d \varepsilon_{k} \frac{\varepsilon+\varepsilon_{k}}{\varepsilon^{2}-\varepsilon_{k}^{2}-\Delta^{2}+i \operatorname{sgn}(\varepsilon) \eta} \\
& =\frac{1}{(2 \pi)^{2} \rho} \frac{2 \pi}{v_{F}^{2}} \int_{-\frac{\omega_{0}}{2}}^{D-\omega_{0} / 2} d \varepsilon_{k}\left[\frac{\varepsilon_{k}\left(\varepsilon+\varepsilon_{k}\right)}{\varepsilon^{2}-\varepsilon_{k}^{2}-\Delta^{2}+i \operatorname{sgn}(\varepsilon) \eta}+\frac{\left.\frac{\left.\omega_{k}\right)}{\varepsilon^{2}-\varepsilon_{k}^{2}-\Delta^{2}+i \operatorname{sgn}(\varepsilon) \eta}\right]}{\frac{\omega_{0}}{2}\left(\varepsilon+\varepsilon_{k}\right)}\right] \\
& =-\frac{1}{(2 \pi)^{2} \rho} \frac{2 \pi}{v_{F}^{2}} \int_{-\frac{\omega_{0}}{2}}^{D-\omega_{0} / 2} d \varepsilon_{k}\left[\frac{\varepsilon_{k}\left(\varepsilon+\varepsilon_{k}\right)}{\varepsilon_{k}^{2}-\varepsilon^{2}+\Delta^{2}-i \operatorname{sgn}(\varepsilon) \eta}+\frac{\omega_{k}^{2}-\varepsilon^{2}+\Delta^{2}-i \operatorname{sgn}(\varepsilon) \eta}{\varepsilon^{2}}\right]
\end{aligned}
$$




$$
\begin{aligned}
g_{--}^{\tau \tau, r} & =\frac{1}{N} \sum_{\mathbf{k}} \frac{\varepsilon-\widetilde{\epsilon}_{+\mathbf{k}}}{\left(\varepsilon-\widetilde{\epsilon}_{+\mathbf{k}}\right)\left(\varepsilon-\widetilde{\epsilon}_{-\mathbf{k}}\right)-\Delta^{2}} \\
& =\frac{S}{(2 \pi)^{2} N} \int k d k d \phi \frac{\varepsilon-\left(v_{F} k-\frac{\omega_{0}}{2}\right)}{\varepsilon^{2}-\left(v_{F} k-\frac{\omega_{0}}{2}\right)^{2}-\Delta^{2}} \\
& =\frac{1}{(2 \pi)^{2} \rho} \frac{2 \pi}{v_{F}^{2}} \int_{-\frac{\omega_{0}}{2}}^{D-\omega_{0} / 2}\left(\varepsilon_{k}+\frac{\omega_{0}}{2}\right) d \varepsilon_{k} \frac{\varepsilon-\varepsilon_{k}}{\varepsilon^{2}-\varepsilon_{k}^{2}-\Delta^{2}+i \operatorname{sgn}(\varepsilon) \eta} \\
& =\frac{1}{(2 \pi)^{2} \rho} \frac{2 \pi}{v_{F}^{2}} \int_{-\frac{\omega_{0}}{2}}^{D-\omega_{0} / 2} d \varepsilon_{k}\left[\frac{\varepsilon_{k}\left(\varepsilon-\varepsilon_{k}\right)}{\varepsilon^{2}-\varepsilon_{k}^{2}-\Delta^{2}+i \operatorname{sgn}(\varepsilon) \eta}+\frac{\frac{\omega_{k}}{2}\left(\varepsilon-\varepsilon_{k}\right)}{\varepsilon^{2}-\varepsilon_{k}^{2}-\Delta^{2}+i \operatorname{sgn}(\varepsilon) \eta}\right] \\
& =-\frac{1}{(2 \pi)^{2} \rho} \frac{2 \pi}{v_{F}^{2}} \int_{-\frac{\omega_{0}}{2}}^{D-\omega_{0} / 2} d \varepsilon_{k}\left[\frac{\varepsilon_{k}\left(\varepsilon-\varepsilon_{k}\right)}{\varepsilon_{k}^{2}-\varepsilon^{2}+\Delta^{2}-i \operatorname{sgn}(\varepsilon) \eta}+\frac{\frac{\omega_{0}}{2}\left(\varepsilon-\varepsilon_{k}\right)}{\varepsilon_{k}^{2}-\varepsilon^{2}+\Delta^{2}-i \operatorname{sgn}(\varepsilon) \eta}\right] \\
g_{+-}^{\tau \tau, r} & =g_{-+}^{\tau \tau, r}=\frac{1}{N} \sum_{\mathbf{k}} \frac{\Delta}{\left(\varepsilon-\widetilde{\epsilon}_{+\mathbf{k}}\right)\left(\varepsilon-\widetilde{\epsilon}_{-\mathbf{k}}\right)-\Delta^{2}} \\
& =\frac{S}{(2 \pi)^{2} N} \int k d k d \phi \frac{\Delta}{\varepsilon^{2}-\left(v_{F} k-\frac{\omega_{0}}{2}\right)^{2}-\Delta^{2}} \\
& =\frac{1}{(2 \pi)^{2} \rho} \frac{2 \pi}{v_{F}^{2}} \int_{-\frac{\omega_{0}}{2}}^{D-\omega_{0} / 2}\left(\varepsilon_{k}+\frac{\omega_{0}}{2}\right) d \varepsilon_{k} \frac{\Delta}{\varepsilon^{2}-\varepsilon_{k}^{2}-\Delta^{2}+i \operatorname{sgn}(\varepsilon) \eta} \\
& =\frac{1}{(2 \pi)^{2} \rho} \frac{2 \pi}{v_{F}^{2}} \int_{-\frac{\omega_{0}}{2}}^{D-\omega_{0} / 2} d \varepsilon_{k}\left[\frac{\omega_{0} \Delta}{\varepsilon^{2}-\varepsilon_{k}^{2}-\Delta^{2}+i \operatorname{sgn}(\varepsilon) \eta}+\frac{\varepsilon_{k} \Delta}{\varepsilon^{2}-\varepsilon_{k}^{2}-\Delta^{2}+i \operatorname{sgn}(\varepsilon) \eta}\right] \\
& =-\frac{1}{2 \pi \rho v_{F}^{2}} \int_{-\frac{\omega_{0}}{2}}^{D-\omega_{0} / 2} d \varepsilon_{k}\left[\frac{\left.\frac{\omega_{0} \Delta}{\varepsilon_{k}^{2}-\varepsilon^{2}+\Delta^{2}-i \operatorname{sgn}(\varepsilon) \eta}+\frac{\omega_{k}^{2}-\varepsilon^{2}+\Delta^{2}-i \operatorname{sgn}(\varepsilon) \eta}{\varepsilon^{2}}\right]}{}\right.
\end{aligned}
$$

Since

$$
\begin{aligned}
& -\frac{1}{2 \pi \rho v_{F}^{2}} \int_{-\frac{\omega_{0}}{2}}^{D-\omega_{0} / 2} d \varepsilon_{k} \frac{\varepsilon_{k}\left(\varepsilon+\varepsilon_{k}\right)}{\left[\varepsilon_{k}-\sqrt{\varepsilon^{2}-\Delta^{2}}-i \operatorname{sgn}(\varepsilon) \eta\right]\left[\varepsilon_{k}+\sqrt{\varepsilon^{2}-\Delta^{2}}+i \operatorname{sgn}(\varepsilon) \eta\right]} \\
& =-\frac{1}{4 \pi \rho v_{F}^{2}} \int_{-\frac{\omega_{0}}{2}}^{D-\omega_{0} / 2} d \varepsilon_{k}\left\{\frac{\varepsilon+\varepsilon_{k}}{\varepsilon_{k}+\sqrt{\varepsilon^{2}-\Delta^{2}}+i \operatorname{sgn}(\varepsilon) \eta}+\frac{\varepsilon+\varepsilon_{k}}{\varepsilon_{k}-\sqrt{\varepsilon^{2}-\Delta^{2}}-i \operatorname{sgn}(\varepsilon) \eta}\right\} \\
& =-\frac{1}{4 \pi \rho v_{F}^{2}} \int_{-\frac{\omega_{0}}{2}}^{D-\omega_{0} / 2} d \varepsilon_{k}\left\{\frac{\varepsilon+\varepsilon_{k}}{\varepsilon_{k}+\sqrt{\varepsilon^{2}-\Delta^{2}}}+\frac{\varepsilon+\varepsilon_{k}}{\varepsilon_{k}-\sqrt{\varepsilon^{2}-\Delta^{2}}}\right\} \\
& -\frac{1}{4 \pi \rho v_{F}^{2}}\left\{-i \pi \operatorname{sgn}(\varepsilon) \theta\left(\Delta<|\varepsilon|<\sqrt{\frac{\omega_{0}^{2}}{4}+\Delta^{2}}\right)\left(\varepsilon-\sqrt{\varepsilon^{2}-\Delta^{2}}\right)\right. \\
& \left.+i \pi \operatorname{sgn}(\varepsilon) \theta\left(\Delta<|\varepsilon|<\sqrt{\left(D-\frac{\omega_{0}}{2}\right)^{2}+\Delta^{2}}\right)\left(\varepsilon+\sqrt{\varepsilon^{2}-\Delta^{2}}\right)\right\} \\
& =-\frac{1}{4 \pi \rho v_{F}^{2}} \int_{-\frac{\omega_{0}}{2}}^{D-\omega_{0} / 2} d \varepsilon_{k}\left\{\frac{\varepsilon+\varepsilon_{k}}{\varepsilon_{k}+\sqrt{\varepsilon^{2}-\Delta^{2}}}+\frac{\varepsilon+\varepsilon_{k}}{\varepsilon_{k}-\sqrt{\varepsilon^{2}-\Delta^{2}}}\right\} \\
& +\frac{1}{4 \pi \rho v_{F}^{2}} i \pi \operatorname{sgn}(\varepsilon)\left\{\theta\left(\Delta<|\varepsilon|<\sqrt{\frac{\omega_{0}^{2}}{4}+\Delta^{2}}\right)\left(\varepsilon-\sqrt{\varepsilon^{2}-\Delta^{2}}\right)\right. \\
& \left.-\theta\left(\Delta<|\varepsilon|<\sqrt{\left(D-\frac{\omega_{0}}{2}\right)^{2}+\Delta^{2}}\right)\left(\varepsilon+\sqrt{\varepsilon^{2}-\Delta^{2}}\right)\right\} \\
& =-\frac{1}{4 \pi \rho v_{F}^{2}}\left[\varepsilon \ln \left|\frac{\left(D-\omega_{0} / 2\right)^{2}-\varepsilon^{2}+\Delta^{2}}{\varepsilon^{2}-\Delta^{2}-\omega_{0}^{2} / 4}\right|-\sqrt{\varepsilon^{2}-\Delta^{2}} \ln \left|\frac{\left(D-\omega_{0} / 2+\sqrt{\varepsilon^{2}-\Delta^{2}}\right)\left(\omega_{0} / 2+\sqrt{\varepsilon^{2}-\Delta^{2}}\right)}{\left(D-\omega_{0} / 2-\sqrt{\varepsilon^{2}-\Delta^{2}}\right)\left(\omega_{0} / 2-\sqrt{\varepsilon^{2}-\Delta^{2}}\right)}\right|+2 D\right] \\
& +\frac{1}{4 \pi \rho v_{F}^{2}} i \pi \operatorname{sgn}(\varepsilon)\left\{\theta\left(\Delta<|\varepsilon|<\sqrt{\frac{\omega_{0}^{2}}{4}+\Delta^{2}}\right)\left(\varepsilon-\sqrt{\varepsilon^{2}-\Delta^{2}}\right)\right. \\
& \left.-\theta\left(\Delta<|\varepsilon|<\sqrt{\left(D-\frac{\omega_{0}}{2}\right)^{2}+\Delta^{2}}\right)\left(\varepsilon+\sqrt{\varepsilon^{2}-\Delta^{2}}\right)\right\} \text {. }
\end{aligned}
$$




$$
\begin{aligned}
& -\frac{1}{2 \pi \rho v_{F}^{2}} \int_{-\frac{\omega_{0}}{2}}^{D-\omega_{0} / 2} d \varepsilon_{k} \frac{\frac{\omega_{0}}{2}\left(\varepsilon+\varepsilon_{k}\right)}{\left[\varepsilon_{k}-\sqrt{\varepsilon^{2}-\Delta^{2}}-i \operatorname{sgn}(\varepsilon) \eta\right]\left[\varepsilon_{k}+\sqrt{\varepsilon^{2}-\Delta^{2}}+i \operatorname{sgn}(\varepsilon) \eta\right]} \\
& =-\frac{1}{4 \pi \rho v_{F}^{2}} \int_{-\frac{\omega_{0}}{2}}^{D-\omega_{0} / 2} d \varepsilon_{k} \frac{-\frac{\omega_{0}}{2}}{\sqrt{\varepsilon^{2}-\Delta^{2}}}\left\{\frac{\varepsilon+\varepsilon_{k}}{\varepsilon_{k}+\sqrt{\varepsilon^{2}-\Delta^{2}}+i \operatorname{sgn}(\varepsilon) \eta}-\frac{\varepsilon+\varepsilon_{k}}{\varepsilon_{k}-\sqrt{\varepsilon^{2}-\Delta^{2}}-i \operatorname{sgn}(\varepsilon) \eta}\right\} \\
& =-\frac{1}{4 \pi \rho v_{F}^{2}} \int_{-\frac{\omega_{0}}{2}}^{D-\omega_{0} / 2} d \varepsilon_{k} \frac{-\frac{\omega_{0}}{2}}{\sqrt{\varepsilon^{2}-\Delta^{2}}}\left\{\frac{\varepsilon+\varepsilon_{k}}{\varepsilon_{k}+\sqrt{\varepsilon^{2}-\Delta^{2}}}-\frac{\varepsilon+\varepsilon_{k}}{\varepsilon_{k}-\sqrt{\varepsilon^{2}-\Delta^{2}}}\right\} \\
& -\frac{1}{4 \pi \rho v_{F}^{2}} \frac{-\frac{\omega_{0}}{2}}{\sqrt{\varepsilon^{2}-\Delta^{2}}}\left\{-i \pi \operatorname{sgn}(\varepsilon) \theta\left(\Delta<|\varepsilon|<\sqrt{\frac{\omega_{0}^{2}}{4}+\Delta^{2}}\right)\left(\varepsilon-\sqrt{\varepsilon^{2}-\Delta^{2}}\right)\right. \\
& \left.-i \pi \operatorname{sgn}(\varepsilon) \theta\left(\Delta<|\varepsilon|<\sqrt{\left(D-\frac{\omega_{0}}{2}\right)^{2}+\Delta^{2}}\right)\left(\varepsilon+\sqrt{\varepsilon^{2}-\Delta^{2}}\right)\right\} \\
& =\frac{1}{4 \pi \rho v_{F}^{2}} \frac{\frac{\omega_{0}}{2}}{\sqrt{\varepsilon^{2}-\Delta^{2}}} \int_{-\frac{\omega_{0}}{2}}^{D-\omega_{0} / 2} d \varepsilon_{k}\left\{\frac{\varepsilon+\varepsilon_{k}}{\varepsilon_{k}+\sqrt{\varepsilon^{2}-\Delta^{2}}}-\frac{\varepsilon+\varepsilon_{k}}{\varepsilon_{k}-\sqrt{\varepsilon^{2}-\Delta^{2}}}\right\} \\
& +\frac{1}{4 \pi \rho v_{F}^{2}} i \pi \operatorname{sgn}(\varepsilon) \frac{-\frac{\omega_{0}}{2}}{\sqrt{\varepsilon^{2}-\Delta^{2}}}\left\{\theta\left(\Delta<|\varepsilon|<\sqrt{\frac{\omega_{0}^{2}}{4}+\Delta^{2}}\right)\left(\varepsilon-\sqrt{\varepsilon^{2}-\Delta^{2}}\right)\right. \\
& \left.+\theta\left(\Delta<|\varepsilon|<\sqrt{\left(D-\frac{\omega_{0}}{2}\right)^{2}+\Delta^{2}}\right)\left(\varepsilon+\sqrt{\varepsilon^{2}-\Delta^{2}}\right)\right\} \\
& =\frac{1}{4 \pi \rho v_{F}^{2}} \frac{\frac{\omega_{0}}{2}}{\sqrt{\varepsilon^{2}-\Delta^{2}}}\left[\varepsilon \ln \left|\frac{\left(D-\omega_{0} / 2+\sqrt{\varepsilon^{2}-\Delta^{2}}\right)\left(\omega_{0} / 2+\sqrt{\varepsilon^{2}-\Delta^{2}}\right)}{\left(D-\omega_{0} / 2-\sqrt{\varepsilon^{2}-\Delta^{2}}\right)\left(\omega_{0} / 2-\sqrt{\varepsilon^{2}-\Delta^{2}}\right)}\right|-\sqrt{\varepsilon^{2}-\Delta^{2}} \ln \left|\frac{\left(D-\omega_{0} / 2\right)^{2}-\varepsilon^{2}+\Delta^{2}}{\varepsilon^{2}-\Delta^{2}-\omega_{0}^{2} / 4}\right|\right] \\
& -\frac{1}{4 \pi \rho v_{F}^{2}} i \pi \operatorname{sgn}(\varepsilon) \frac{\frac{\omega_{0}}{2}}{\sqrt{\varepsilon^{2}-\Delta^{2}}}\left\{\theta\left(\Delta<|\varepsilon|<\sqrt{\frac{\omega_{0}^{2}}{4}+\Delta^{2}}\right)\left(\varepsilon-\sqrt{\varepsilon^{2}-\Delta^{2}}\right)\right. \\
& \left.+\theta\left(\Delta<|\varepsilon|<\sqrt{\left(D-\frac{\omega_{0}}{2}\right)^{2}+\Delta^{2}}\right)\left(\varepsilon+\sqrt{\varepsilon^{2}-\Delta^{2}}\right)\right\}
\end{aligned}
$$

we obtain

$$
\begin{aligned}
g_{++}^{\tau \tau, r}= & -\frac{1}{4 \pi \rho v_{F}^{2}}\left[\left(\varepsilon+\frac{\frac{\omega_{0}}{2}}{\sqrt{\varepsilon^{2}-\Delta^{2}}} \sqrt{\varepsilon^{2}-\Delta^{2}}\right) \ln \left|\frac{\left(D-\omega_{0} / 2\right)^{2}-\varepsilon^{2}+\Delta^{2}}{\varepsilon^{2}-\Delta^{2}-\omega_{0}^{2} / 4}\right|\right. \\
& \left.-\left(\varepsilon \frac{\frac{\omega_{0}}{\sqrt{\varepsilon^{2}-\Delta^{2}}}}{\sqrt{\varepsilon^{2}-\Delta^{2}}}\right) \ln \left|\frac{\left(D-\omega_{0} / 2+\sqrt{\varepsilon^{2}-\Delta^{2}}\right)\left(\omega_{0} / 2+\sqrt{\varepsilon^{2}-\Delta^{2}}\right)}{\left(D-\omega_{0} / 2-\sqrt{\varepsilon^{2}-\Delta^{2}}\right)\left(\omega_{0} / 2-\sqrt{\varepsilon^{2}-\Delta^{2}}\right)}\right|+2 D\right] \\
& +\frac{1}{4 \pi \rho v_{F}^{2}} i \pi \operatorname{sgn}(\varepsilon)\left\{\theta\left(\Delta<|\varepsilon|<\sqrt{\frac{\omega_{0}^{2}}{4}+\Delta^{2}}\right)\left(\varepsilon-\sqrt{\varepsilon^{2}-\Delta^{2}}\right)\left(1-\frac{\frac{\omega_{0}}{2}}{\sqrt{\varepsilon^{2}-\Delta^{2}}}\right)\right. \\
& \left.-\theta\left(\Delta<|\varepsilon|<\sqrt{\left(D-\frac{\omega_{0}}{2}\right)^{2}+\Delta^{2}}\right)\left(\varepsilon+\sqrt{\varepsilon^{2}-\Delta^{2}}\right)\left(1+\frac{\frac{\omega_{0}}{2}}{\sqrt{\varepsilon^{2}-\Delta^{2}}}\right)\right\} .
\end{aligned}
$$

In order to calculate $g_{--}^{\tau \tau, r}$, we solve at first for

$$
\begin{aligned}
& -\frac{1}{2 \pi \rho v_{F}^{2}} \int_{-\frac{\omega_{0}}{2}}^{D-\omega_{0} / 2} d \varepsilon_{k} \frac{\varepsilon_{k}\left(\varepsilon-\varepsilon_{k}\right)}{\left[\varepsilon_{k}-\sqrt{\varepsilon^{2}-\Delta^{2}}-i \operatorname{sgn}(\varepsilon) \eta\right]\left[\varepsilon_{k}+\sqrt{\varepsilon^{2}-\Delta^{2}}+i \operatorname{sgn}(\varepsilon) \eta\right]} \\
& =-\frac{1}{4 \pi \rho v_{F}^{2}} \int_{-\frac{\omega_{0}}{2}}^{D-\omega_{0} / 2} d \varepsilon_{k}\left\{\frac{\varepsilon-\varepsilon_{k}}{\varepsilon_{k}+\sqrt{\varepsilon^{2}-\Delta^{2}}+i \operatorname{sgn}(\varepsilon) \eta}+\frac{\varepsilon-\varepsilon_{k}}{\varepsilon_{k}-\sqrt{\varepsilon^{2}-\Delta^{2}}-i \operatorname{sgn}(\varepsilon) \eta}\right\} \\
& =-\frac{1}{4 \pi \rho v_{F}^{2}} \int_{-\frac{\omega_{0}}{2}}^{D-\omega_{0} / 2} d \varepsilon_{k}\left\{\frac{\varepsilon-\varepsilon_{k}}{\varepsilon_{k}+\sqrt{\varepsilon^{2}-\Delta^{2}}}+\frac{\varepsilon-\varepsilon_{k}}{\varepsilon_{k}-\sqrt{\varepsilon^{2}-\Delta^{2}}}\right\} \\
& -\frac{1}{4 \pi \rho v_{F}^{2}}\left\{-i \pi \operatorname{sgn}(\varepsilon) \theta\left(\Delta<|\varepsilon|<\sqrt{\frac{\omega_{0}^{2}}{4}+\Delta^{2}}\right)\left(\varepsilon+\sqrt{\varepsilon^{2}-\Delta^{2}}\right)\right. \\
& \left.+i \pi \operatorname{sgn}(\varepsilon) \theta\left(\Delta<|\varepsilon|<\sqrt{\left(D-\frac{\omega_{0}}{2}\right)^{2}+\Delta^{2}}\right)\left(\varepsilon-\sqrt{\varepsilon^{2}-\Delta^{2}}\right)\right\} \\
& =-\frac{1}{4 \pi \rho v_{F}^{2}} \int_{-\frac{\omega_{0}}{2}}^{D-\omega_{0} / 2} d \varepsilon_{k}\left\{\frac{\varepsilon-\varepsilon_{k}}{\varepsilon_{k}+\sqrt{\varepsilon^{2}-\Delta^{2}}}+\frac{\varepsilon-\varepsilon_{k}}{\varepsilon_{k}-\sqrt{\varepsilon^{2}-\Delta^{2}}}\right\} \\
& +\frac{1}{4 \pi \rho v_{F}^{2}} i \pi \operatorname{sgn}(\varepsilon)\left\{\theta\left(\Delta<|\varepsilon|<\sqrt{\frac{\omega_{0}^{2}}{4}+\Delta^{2}}\right)\left(\varepsilon+\sqrt{\varepsilon^{2}-\Delta^{2}}\right)\right. \\
& \left.-\theta\left(\Delta<|\varepsilon|<\sqrt{\left(D-\frac{\omega_{0}}{2}\right)^{2}+\Delta^{2}}\right)\left(\varepsilon-\sqrt{\varepsilon^{2}-\Delta^{2}}\right)\right\} \\
& =-\frac{1}{4 \pi \rho v_{F}^{2}}\left[\varepsilon \ln \left|\frac{\left(D-\omega_{0} / 2\right)^{2}-\varepsilon^{2}+\Delta^{2}}{\varepsilon^{2}-\Delta^{2}-\omega_{0}^{2} / 4}\right|+\sqrt{\varepsilon^{2}-\Delta^{2}} \ln \left|\frac{\left(D-\omega_{0} / 2+\sqrt{\varepsilon^{2}-\Delta^{2}}\right)\left(\omega_{0} / 2+\sqrt{\varepsilon^{2}-\Delta^{2}}\right)}{\left(D-\omega_{0} / 2-\sqrt{\varepsilon^{2}-\Delta^{2}}\right)\left(\omega_{0} / 2-\sqrt{\varepsilon^{2}-\Delta^{2}}\right)}\right|-2 D\right] \\
& +\frac{1}{4 \pi \rho v_{F}^{2}} i \pi \operatorname{sgn}(\varepsilon)\left\{\theta\left(\Delta<|\varepsilon|<\sqrt{\frac{\omega_{0}^{2}}{4}+\Delta^{2}}\right)\left(\varepsilon+\sqrt{\varepsilon^{2}-\Delta^{2}}\right)\right. \\
& \left.-\theta\left(\Delta<|\varepsilon|<\sqrt{\left(D-\frac{\omega_{0}}{2}\right)^{2}+\Delta^{2}}\right)\left(\varepsilon-\sqrt{\varepsilon^{2}-\Delta^{2}}\right)\right\}
\end{aligned}
$$


Laser-assisted spin-polarized transport in graphene tunnel junctions

$$
\begin{aligned}
& -\frac{1}{2 \pi \rho v_{F}^{2}} \int_{-\frac{\omega_{0}}{2}}^{D-\omega_{0} / 2} d \varepsilon_{k} \frac{\frac{\omega_{0}}{2}\left(\varepsilon-\varepsilon_{k}\right)}{\left[\varepsilon_{k}-\sqrt{\varepsilon^{2}-\Delta^{2}}-i \operatorname{sgn}(\varepsilon) \eta\right]\left[\varepsilon_{k}+\sqrt{\varepsilon^{2}-\Delta^{2}}+i \operatorname{sgn}(\varepsilon) \eta\right]} \\
& =-\frac{1}{4 \pi \rho v_{F}^{2}} \int_{-\frac{\omega_{0}}{2}}^{D-\omega_{0} / 2} d \varepsilon_{k} \frac{-\frac{\omega_{0}}{2}}{\sqrt{\varepsilon^{2}-\Delta^{2}}}\left\{\frac{\varepsilon-\varepsilon_{k}}{\varepsilon_{k}+\sqrt{\varepsilon^{2}-\Delta^{2}}+i \operatorname{sgn}(\varepsilon) \eta}-\frac{\varepsilon-\varepsilon_{k}}{\varepsilon_{k}-\sqrt{\varepsilon^{2}-\Delta^{2}}-i \operatorname{sgn}(\varepsilon) \eta}\right\} \\
& =-\frac{1}{4 \pi \rho v_{F}^{2}} \int_{-\frac{\omega_{0}}{2}}^{D-\omega_{0} / 2} d \varepsilon_{k} \frac{-\frac{\omega_{0}}{2}}{\sqrt{\varepsilon^{2}-\Delta^{2}}}\left\{\frac{\varepsilon-\varepsilon_{k}}{\varepsilon_{k}+\sqrt{\varepsilon^{2}-\Delta^{2}}}-\frac{\varepsilon-\varepsilon_{k}}{\varepsilon_{k}-\sqrt{\varepsilon^{2}-\Delta^{2}}}\right\} \\
& -\frac{1}{4 \pi \rho v_{F}^{2}} \frac{-\frac{\omega_{0}}{2}}{\sqrt{\varepsilon^{2}-\Delta^{2}}}\left\{-i \pi \operatorname{sgn}(\varepsilon) \theta\left(\Delta<|\varepsilon|<\sqrt{\frac{\omega_{0}^{2}}{4}+\Delta^{2}}\right)\left(\varepsilon+\sqrt{\varepsilon^{2}-\Delta^{2}}\right)\right. \\
& \left.-i \pi \operatorname{sgn}(\varepsilon) \theta\left(\Delta<|\varepsilon|<\sqrt{\left(D-\frac{\omega_{0}}{2}\right)^{2}+\Delta^{2}}\right)\left(\varepsilon-\sqrt{\varepsilon^{2}-\Delta^{2}}\right)\right\} \\
& =\frac{1}{4 \pi \rho v_{F}^{2}} \frac{\frac{\omega_{0}}{2}}{\sqrt{\varepsilon^{2}-\Delta^{2}}} \int_{-\frac{\omega_{0}}{2}}^{D-\omega_{0} / 2} d \varepsilon_{k}\left\{\frac{\varepsilon-\varepsilon_{k}}{\varepsilon_{k}+\sqrt{\varepsilon^{2}-\Delta^{2}}}-\frac{\varepsilon-\varepsilon_{k}}{\varepsilon_{k}-\sqrt{\varepsilon^{2}-\Delta^{2}}}\right\} \\
& +\frac{1}{4 \pi \rho v_{F}^{2}} i \pi \operatorname{sgn}(\varepsilon) \frac{-\frac{\omega_{0}}{2}}{\sqrt{\varepsilon^{2}-\Delta^{2}}}\left\{\theta\left(\Delta<|\varepsilon|<\sqrt{\frac{\omega_{0}^{2}}{4}+\Delta^{2}}\right)\left(\varepsilon+\sqrt{\varepsilon^{2}-\Delta^{2}}\right)\right. \\
& \left.+\theta\left(\Delta<|\varepsilon|<\sqrt{\left(D-\frac{\omega_{0}}{2}\right)^{2}+\Delta^{2}}\right)\left(\varepsilon-\sqrt{\varepsilon^{2}-\Delta^{2}}\right)\right\} \\
& =\frac{1}{4 \pi \rho v_{F}^{2}} \frac{\frac{\omega_{0}}{2}}{\sqrt{\varepsilon^{2}-\Delta^{2}}}\left[\varepsilon \ln \left|\frac{\left(D-\omega_{0} / 2+\sqrt{\varepsilon^{2}-\Delta^{2}}\right)\left(\omega_{0} / 2+\sqrt{\varepsilon^{2}-\Delta^{2}}\right)}{\left(D-\omega_{0} / 2-\sqrt{\varepsilon^{2}-\Delta^{2}}\right)\left(\omega_{0} / 2-\sqrt{\varepsilon^{2}-\Delta^{2}}\right)}\right|+\sqrt{\varepsilon^{2}-\Delta^{2}} \ln \left|\frac{\left(D-\omega_{0} / 2\right)^{2}-\varepsilon^{2}+\Delta^{2}}{\varepsilon^{2}-\Delta^{2}-\omega_{0}^{2} / 4}\right|\right] \\
& -\frac{1}{4 \pi \rho v_{F}^{2}} i \pi \operatorname{sgn}(\varepsilon) \frac{\frac{\omega_{0}}{2}}{\sqrt{\varepsilon^{2}-\Delta^{2}}}\left\{\theta\left(\Delta<|\varepsilon|<\sqrt{\frac{\omega_{0}^{2}}{4}+\Delta^{2}}\right)\left(\varepsilon+\sqrt{\varepsilon^{2}-\Delta^{2}}\right)\right. \\
& \left.+\theta\left(\Delta<|\varepsilon|<\sqrt{\left(D-\frac{\omega_{0}}{2}\right)^{2}+\Delta^{2}}\right)\left(\varepsilon-\sqrt{\varepsilon^{2}-\Delta^{2}}\right)\right\} \text {, }
\end{aligned}
$$

This means that

$$
\begin{aligned}
g_{--}^{\tau \tau, r}= & -\frac{1}{4 \pi \rho v_{F}^{2}}\left[\left(\varepsilon-\frac{\frac{\omega_{0}}{2}}{\sqrt{\varepsilon^{2}-\Delta^{2}}} \sqrt{\varepsilon^{2}-\Delta^{2}}\right) \ln \left|\frac{\left(D-\omega_{0} / 2\right)^{2}-\varepsilon^{2}+\Delta^{2}}{\varepsilon^{2}-\Delta^{2}-\omega_{0}^{2} / 4}\right|\right. \\
& \left.+\left(\sqrt{\varepsilon^{2}-\Delta^{2}}-\varepsilon \frac{\frac{\omega_{0}}{2}}{\sqrt{\varepsilon^{2}-\Delta^{2}}}\right) \ln \left|\frac{\left(D-\omega_{0} / 2+\sqrt{\varepsilon^{2}-\Delta^{2}}\right)\left(\omega_{0} / 2+\sqrt{\varepsilon^{2}-\Delta^{2}}\right)}{\left(D-\omega_{0} / 2-\sqrt{\varepsilon^{2}-\Delta^{2}}\right)\left(\omega_{0} / 2-\sqrt{\varepsilon^{2}-\Delta^{2}}\right)}\right|-2 D\right] \\
& +\frac{1}{4 \pi \rho v_{F}^{2}} i \pi \operatorname{sgn}(\varepsilon)\left\{\theta\left(\Delta<|\varepsilon|<\sqrt{\frac{\omega_{0}^{2}}{4}+\Delta^{2}}\right)\left(\varepsilon+\sqrt{\varepsilon^{2}-\Delta^{2}}\right)\left(1-\frac{\frac{\omega_{0}}{2}}{\sqrt{\varepsilon^{2}-\Delta^{2}}}\right)\right. \\
& \left.-\theta\left(\Delta<|\varepsilon|<\sqrt{\left(D-\frac{\omega_{0}}{2}\right)^{2}+\Delta^{2}}\right)\left(\varepsilon-\sqrt{\varepsilon^{2}-\Delta^{2}}\right)\left(1+\frac{\frac{\omega_{0}}{2}}{\sqrt{\varepsilon^{2}-\Delta^{2}}}\right)\right\} .
\end{aligned}
$$

Similar to the calculations of the Green's functions $g_{++}^{\tau \tau, r}$ and $g_{--}^{\tau \tau, r}$, we consider at first the following terms in $g_{+-}^{\tau \tau, r}$,

$$
\begin{aligned}
& -\frac{1}{2 \pi \rho v_{F}^{2}} \int_{-\frac{\omega_{0}}{2}}^{D-\omega_{0} / 2} d \varepsilon_{k} \frac{\varepsilon_{k} \Delta}{\left[\varepsilon_{k}-\sqrt{\varepsilon^{2}-\Delta^{2}}-i \operatorname{sgn}(\varepsilon) \eta\right]\left[\varepsilon_{k}+\sqrt{\varepsilon^{2}-\Delta^{2}}+i \operatorname{sgn}(\varepsilon) \eta\right]} \\
= & -\frac{1}{4 \pi \rho v_{F}^{2}} \int_{-\frac{\omega_{0}}{2}}^{D-\omega_{0} / 2} d \varepsilon_{k}\left\{\frac{\Delta}{\varepsilon_{k}+\sqrt{\varepsilon^{2}-\Delta^{2}}+i \operatorname{sgn}(\varepsilon) \eta}+\frac{\Delta}{\varepsilon_{k}-\sqrt{\varepsilon^{2}-\Delta^{2}}-i \operatorname{sgn}(\varepsilon) \eta}\right\} \\
= & -\frac{1}{4 \pi \rho v_{F}^{2}} \int_{-\frac{\omega_{0}}{2}}^{D-\omega_{0} / 2} d \varepsilon_{k}\left\{\frac{\Delta}{\varepsilon_{k}+\sqrt{\varepsilon^{2}-\Delta^{2}}}+\frac{\Delta}{\varepsilon_{k}-\sqrt{\varepsilon^{2}-\Delta^{2}}}\right\} \\
& -\frac{1}{4 \pi \rho v_{F}^{2}}\left\{-i \pi \operatorname{sgn}(\varepsilon) \theta\left(\Delta<|\varepsilon|<\sqrt{\frac{\omega_{0}^{2}}{4}+\Delta^{2}}\right) \Delta\right. \\
& \left.+i \pi \operatorname{sgn}(\varepsilon) \theta\left(\Delta<|\varepsilon|<\sqrt{\left(D-\frac{\omega_{0}}{2}\right)^{2}+\Delta^{2}}\right) \Delta\right\} \\
= & -\frac{1}{4 \pi \rho v_{F}^{2}} \int_{-\frac{\omega_{0}}{2}}^{D-\omega_{0} / 2} d \varepsilon_{k}\left\{\frac{\Delta}{\varepsilon_{k}+\sqrt{\varepsilon^{2}-\Delta^{2}}}+\frac{\Delta}{\varepsilon_{k}-\sqrt{\varepsilon^{2}-\Delta^{2}}}\right\} \\
& +\frac{\Delta}{4 \pi \rho v_{F}^{2}} i \pi \operatorname{sgn}(\varepsilon)\left\{\theta\left(\Delta<|\varepsilon|<\sqrt{\frac{\omega_{0}^{2}}{4}+\Delta^{2}}\right)-\theta\left(\Delta<|\varepsilon|<\sqrt{\left(D-\frac{\omega_{0}}{2}\right)^{2}+\Delta^{2}}\right)\right\} \\
= & -\frac{\Delta}{4 \pi \rho v_{F}^{2}} \ln \left|\frac{\left(D-\omega_{0} / 2\right)^{2}-\varepsilon^{2}+\Delta^{2}}{\varepsilon^{2}-\Delta^{2}-\omega_{0}^{2} / 4}\right| \\
& +\frac{\Delta}{4 \pi \rho v_{F}^{2}} i \pi \operatorname{sgn}(\varepsilon)\left\{\theta\left(\Delta<|\varepsilon|<\sqrt{\frac{\omega_{0}^{2}}{4}+\Delta^{2}}\right)-\theta\left(\Delta<|\varepsilon|<\sqrt{\left(D-\frac{\omega_{0}}{2}\right)^{2}+\Delta^{2}}\right)\right\}
\end{aligned}
$$




$$
\begin{aligned}
& -\frac{1}{2 \pi \rho v_{F}^{2}} \int_{-\frac{\omega_{0}}{2}}^{D-\omega_{0} / 2} d \varepsilon_{k} \frac{\frac{\omega_{0}}{2} \Delta}{\left[\varepsilon_{k}-\sqrt{\varepsilon^{2}-\Delta^{2}}-i \operatorname{sgn}(\varepsilon) \eta\right]\left[\varepsilon_{k}+\sqrt{\varepsilon^{2}-\Delta^{2}}+i \operatorname{sgn}(\varepsilon) \eta\right]} \\
& =-\frac{1}{4 \pi \rho v_{F}^{2}} \int_{-\frac{\omega_{0}}{2}}^{D-\omega_{0} / 2} d \varepsilon_{k} \frac{-\frac{\omega_{0}}{2}}{\sqrt{\varepsilon^{2}-\Delta^{2}}}\left\{\frac{\Delta}{\varepsilon_{k}+\sqrt{\varepsilon^{2}-\Delta^{2}}+i \operatorname{sgn}(\varepsilon) \eta}-\frac{\Delta}{\varepsilon_{k}-\sqrt{\varepsilon^{2}-\Delta^{2}}-i \operatorname{sgn}(\varepsilon) \eta}\right\} \\
& =-\frac{1}{4 \pi \rho v_{F}^{2}} \int_{-\frac{\omega_{0}}{2}}^{D-\omega_{0} / 2} d \varepsilon_{k} \frac{-\frac{\omega_{0}}{2}}{\sqrt{\varepsilon^{2}-\Delta^{2}}}\left\{\frac{\Delta}{\varepsilon_{k}+\sqrt{\varepsilon^{2}-\Delta^{2}}}-\frac{\Delta}{\varepsilon_{k}-\sqrt{\varepsilon^{2}-\Delta^{2}}}\right\} \\
& -\frac{1}{4 \pi \rho v_{F}^{2}} \frac{-\frac{\omega_{0}}{2}}{\sqrt{\varepsilon^{2}-\Delta^{2}}}\left\{-i \pi \operatorname{sgn}(\varepsilon) \theta\left(\Delta<|\varepsilon|<\sqrt{\frac{\omega_{0}^{2}}{4}+\Delta^{2}}\right) \Delta\right. \\
& \left.-i \pi \operatorname{sgn}(\varepsilon) \theta\left(\Delta<|\varepsilon|<\sqrt{\left(D-\frac{\omega_{0}}{2}\right)^{2}+\Delta^{2}}\right) \Delta\right\} \\
& =\frac{1}{4 \pi \rho v_{F}^{2}} \frac{\frac{\omega_{0}}{2}}{\sqrt{\varepsilon^{2}-\Delta^{2}}} \int_{-\frac{\omega_{0}}{2}}^{D-\omega_{0} / 2} d \varepsilon_{k}\left\{\frac{\Delta}{\varepsilon_{k}+\sqrt{\varepsilon^{2}-\Delta^{2}}}-\frac{\Delta}{\varepsilon_{k}-\sqrt{\varepsilon^{2}-\Delta^{2}}}\right\} \\
& +\frac{1}{4 \pi \rho v_{F}^{2}} i \pi \operatorname{sgn}(\varepsilon) \frac{-\frac{\omega_{0}}{2} \Delta}{\sqrt{\varepsilon^{2}-\Delta^{2}}}\left\{\theta\left(\Delta<|\varepsilon|<\sqrt{\frac{\omega_{0}^{2}}{4}+\Delta^{2}}\right)+\theta\left(\Delta<|\varepsilon|<\sqrt{\left(D-\frac{\omega_{0}}{2}\right)^{2}+\Delta^{2}}\right)\right\} \\
& =\frac{1}{4 \pi \rho v_{F}^{2}} \frac{\frac{\omega_{0}}{2} \Delta}{\sqrt{\varepsilon^{2}-\Delta^{2}}} \ln \left|\frac{\left(D-\omega_{0} / 2+\sqrt{\varepsilon^{2}-\Delta^{2}}\right)\left(\omega_{0} / 2+\sqrt{\varepsilon^{2}-\Delta^{2}}\right)}{\left(D-\omega_{0} / 2-\sqrt{\varepsilon^{2}-\Delta^{2}}\right)\left(\omega_{0} / 2-\sqrt{\varepsilon^{2}-\Delta^{2}}\right)}\right| \\
& -\frac{1}{4 \pi \rho v_{F}^{2}} i \pi \operatorname{sgn}(\varepsilon) \frac{\frac{\omega_{0}}{2} \Delta}{\sqrt{\varepsilon^{2}-\Delta^{2}}}\left\{\theta\left(\Delta<|\varepsilon|<\sqrt{\frac{\omega_{0}^{2}}{4}+\Delta^{2}}\right)+\theta\left(\Delta<|\varepsilon|<\sqrt{\left(D-\frac{\omega_{0}}{2}\right)^{2}+\Delta^{2}}\right)\right\}, \\
& g_{+-}^{\tau \tau, r} \quad=g_{-+}^{\tau \tau, r} \\
& =-\frac{\Delta}{4 \pi \rho v_{F}^{2}} \ln \left|\frac{\left(D-\omega_{0} / 2\right)^{2}-\varepsilon^{2}+\Delta^{2}}{\varepsilon^{2}-\Delta^{2}-\omega_{0}^{2} / 4}\right| \\
& +\frac{\Delta}{4 \pi \rho v_{F}^{2}} i \pi \operatorname{sgn}(\varepsilon)\left\{\theta\left(\Delta<|\varepsilon|<\sqrt{\frac{\omega_{0}^{2}}{4}+\Delta^{2}}\right)-\theta\left(\Delta<|\varepsilon|<\sqrt{\left(D-\frac{\omega_{0}}{2}\right)^{2}+\Delta^{2}}\right)\right\} \\
& +\frac{1}{4 \pi \rho v_{F}^{2}} \frac{\frac{\omega_{0}}{2} \Delta}{\sqrt{\varepsilon^{2}-\Delta^{2}}} \ln \left|\frac{\left(D-\omega_{0} / 2+\sqrt{\varepsilon^{2}-\Delta^{2}}\right)\left(\omega_{0} / 2+\sqrt{\varepsilon^{2}-\Delta^{2}}\right)}{\left(D-\omega_{0} / 2-\sqrt{\varepsilon^{2}-\Delta^{2}}\right)\left(\omega_{0} / 2-\sqrt{\varepsilon^{2}-\Delta^{2}}\right)}\right| \\
& -\frac{\Delta}{4 \pi \rho v_{F}^{2}} i \pi \operatorname{sgn}(\varepsilon) \frac{\frac{\omega_{0}}{2}}{\sqrt{\varepsilon^{2}-\Delta^{2}}}\left\{\theta\left(\Delta<|\varepsilon|<\sqrt{\frac{\omega_{0}^{2}}{4}+\Delta^{2}}\right)+\theta\left(\Delta<|\varepsilon|<\sqrt{\left(D-\frac{\omega_{0}}{2}\right)^{2}+\Delta^{2}}\right)\right\} \\
& =-\frac{\Delta}{4 \pi \rho v_{F}^{2}}\left[\ln \left|\frac{\left(D-\omega_{0} / 2\right)^{2}-\varepsilon^{2}+\Delta^{2}}{\varepsilon^{2}-\Delta^{2}-\omega_{0}^{2} / 4}\right|-\frac{\frac{\omega_{0}}{2}}{\sqrt{\varepsilon^{2}-\Delta^{2}}} \ln \left|\frac{\left(D-\omega_{0} / 2+\sqrt{\varepsilon^{2}-\Delta^{2}}\right)\left(\omega_{0} / 2+\sqrt{\varepsilon^{2}-\Delta^{2}}\right)}{\left(D-\omega_{0} / 2-\sqrt{\varepsilon^{2}-\Delta^{2}}\right)\left(\omega_{0} / 2-\sqrt{\varepsilon^{2}-\Delta^{2}}\right)}\right|\right] \\
& +\frac{\Delta}{4 \pi \rho v_{F}^{2}} i \pi \operatorname{sgn}(\varepsilon)\left\{\theta\left(\Delta<|\varepsilon|<\sqrt{\frac{\omega_{0}^{2}}{4}+\Delta^{2}}\right)\left(1-\frac{\frac{\omega_{0}}{2}}{\sqrt{\varepsilon^{2}-\Delta^{2}}}\right)\right. \\
& \left.-\theta\left(\Delta<|\varepsilon|<\sqrt{\left(D-\frac{\omega_{0}}{2}\right)^{2}+\Delta^{2}}\right)\left(1+\frac{\frac{\omega_{0}}{2}}{\sqrt{\varepsilon^{2}-\Delta^{2}}}\right)\right\} \text {. }
\end{aligned}
$$

\section{Appendix D.3. Fourier-transformed Green's functions}

Since $H(t+T)=H(t)$ applies, the retarded Green's function obeys $\tilde{G}\left(t, t^{\prime}\right)=$ $\tilde{G}\left(t+T, t^{\prime}+T\right)$ (S. Kohler, et al.,Physics Reports 406, 379(2005)). As we can write $\tilde{G}\left(t, t^{\prime}\right)=\tilde{G}\left(t+T, t^{\prime}+T\right)=\tilde{G}\left(t+T, t+T-\left(t-t^{\prime}\right)\right)$ we define $\tau=t$ and $\tau^{\prime}=t-t^{\prime}$ and express the Green's functions as $\tilde{G}\left(t+T, t+T-\left(t-t^{\prime}\right)\right) \equiv G\left(\tau+T, \tau^{\prime}\right)$, which satisfies $G\left(\tau+T, \tau^{\prime}\right)=G\left(\tau, \tau^{\prime}\right)$. Thus, one can introduce the Fourier transformation

$$
G\left(\tau, \tau^{\prime}\right)=\sum_{n} e^{i \frac{n \omega_{0} \tau}{2}} \int \frac{d \varepsilon}{2 \pi} e^{-i \varepsilon \tau^{\prime}} G_{n}(\varepsilon)
$$

which is the same as the generalized Fourier form

$$
\begin{aligned}
G\left(t, t^{\prime}\right) & =\frac{1}{2 \pi} \sum_{n} \int d \varepsilon e^{-i \varepsilon t} e^{i\left(\varepsilon+n \omega_{0} / 2\right) t^{\prime}} G\left(\varepsilon, \varepsilon+n \omega_{0} / 2\right)=\sum_{n} e^{i \frac{n \omega_{0} t}{2}} \int \frac{d \varepsilon}{2 \pi} e^{-i\left(\varepsilon+n \omega_{0} / 2\right)\left(t-t^{\prime}\right)} G\left(\varepsilon, \varepsilon+n \omega_{0} / 2\right) \\
& =\sum_{n} e^{i \frac{n \omega_{0} t}{2}} \int \frac{d \varepsilon}{2 \pi} e^{-i \varepsilon\left(t-t^{\prime}\right)} G\left(\varepsilon-n \omega_{0} / 2, \varepsilon\right)
\end{aligned}
$$

For $\tau=t$ and $\tau^{\prime}=t-t^{\prime}$ same expression as Eq. (D.29) follows.

Denoting $G_{m n}(\varepsilon)=G\left(\varepsilon+m \omega_{0} / 2, \varepsilon+n \omega_{0} / 2\right)$ allows to write for the different Fourier components $G_{m n}(\varepsilon)=G_{m-n, 0}\left(\varepsilon+n \omega_{0} / 2\right)$, for $G_{m n}(\varepsilon)=G\left(\varepsilon+m \omega_{0} / 2, \varepsilon+n \omega_{0} / 2\right)=$ 
$G\left[\varepsilon+n \omega_{0} / 2+(m-n) \omega_{0} / 2, \varepsilon+n \omega_{0} / 2\right]=G_{m-n, 0}\left(\varepsilon+n \omega_{0} / 2\right)$ applies. For the inverse transformation of Eq.(D.29) one finds

$$
G_{m n}(\varepsilon)=\frac{1}{T} \int d t d t^{\prime} e^{i\left(m \omega_{0} t / 2-n \omega_{0} t^{\prime} / 2\right)} e^{i \varepsilon\left(t-t^{\prime}\right)} G\left(t, t^{\prime}\right) .
$$

For the Fourier transformation of the Green's function one finds

$$
\begin{aligned}
g_{m n}(\varepsilon) & =\frac{1}{T} \int d t d t^{\prime} e^{i\left(m \omega_{0} t / 2-n \omega_{0} t^{\prime} / 2\right)} e^{i \varepsilon\left(t-t^{\prime}\right)} g\left(t, t^{\prime}\right) \\
& =\frac{1}{T} \int d t d t^{\prime} \int \frac{d \varepsilon^{\prime}}{2 \pi} e^{i\left(m \omega_{0} t / 2-n \omega_{0} t^{\prime} / 2\right)} e^{i \varepsilon\left(t-t^{\prime}\right)} e^{-i \varepsilon^{\prime}\left(t-t^{\prime}\right)} g\left(\varepsilon^{\prime}\right) \\
& =\frac{1}{T} \int_{0}^{T} d t \int \frac{d \varepsilon^{\prime}}{2 \pi} e^{i(m-n) \omega_{0} t / 2} g\left(\varepsilon^{\prime}\right) \int_{-\infty}^{\infty} d t^{\prime} e^{i\left(\varepsilon-\varepsilon^{\prime}+n \omega_{0} / 2\right)\left(t-t^{\prime}\right)} \\
& =\frac{1}{T} \int_{0}^{T} d t \int d \varepsilon^{\prime} e^{i(m-n) \omega_{0} t / 2} g\left(\varepsilon^{\prime}\right) \delta\left(\varepsilon-\varepsilon^{\prime}+n \omega_{0} / 2\right) \\
& =\frac{1}{T} \int_{0}^{T} d t e^{i(m-n) \omega_{0} t / 2} g\left(\varepsilon+n \omega_{0} / 2\right) \\
& =\left\{\begin{array}{l}
g\left(\varepsilon+n \omega_{0} / 2\right), \text { for } m=n \\
g\left(\varepsilon+n \omega_{0} / 2\right) \frac{1}{i(m-n) T \omega_{0} / 2}\left[e^{i(m-n) \omega_{0} T / 2}-1\right]=0, \text { for } m \neq n \\
=
\end{array}\right.
\end{aligned}
$$

For the selfenergy we write

$$
\begin{aligned}
\Sigma_{m n}^{\tau, r}(\varepsilon) & =\frac{1}{T} \int \frac{d \varepsilon^{\prime}}{2 \pi} \int d t d t^{\prime} e^{i\left(m \omega_{0} t / 2-n \omega_{0} t^{\prime} / 2\right)} e^{i \varepsilon\left(t-t^{\prime}\right)} \Sigma^{\tau, r} e^{-i \varepsilon^{\prime}\left(t-t^{\prime}\right)}\left(\begin{array}{cc}
e^{i \frac{\omega_{0}}{2}\left(t-t^{\prime}\right)} & e^{i \frac{\omega_{0}}{2}\left(t+t^{\prime}\right)} \\
e^{-i \frac{\omega_{0}}{2}\left(t+t^{\prime}\right)} & e^{-i \frac{\omega_{0}}{2}\left(t-t^{\prime}\right)}
\end{array}\right) \\
& =\Sigma^{\tau, r}\left(\begin{array}{cc}
\delta_{m n} & \delta_{n, m+2} \\
\delta_{n, m-2} & \delta_{n, m}
\end{array}\right) .
\end{aligned}
$$

The last terms of Eq.(D.10) can be cast as

$$
\begin{aligned}
& \int d t_{1} \int d t_{2} g^{\tau \tau, r}\left(t-t_{1}\right) \Sigma^{\tau, r}\left(t_{1}, t_{2}\right) G^{\tau \tau^{\prime}, r}\left(t_{2}, t^{\prime}\right) \\
& =\frac{1}{(2 \pi)^{3}} \int d t_{1} d t_{2} \sum_{n_{1} n_{2} n_{3}} \int d \varepsilon_{1} d \varepsilon_{2} d \varepsilon_{3} e^{-i\left(\varepsilon_{1}+\frac{m_{1} \omega_{0}}{2}\right) t} e^{i\left(\varepsilon_{1}+\frac{n_{1} \omega_{0}}{2}\right) t_{1}} e^{-i\left(\varepsilon_{2}+\frac{m_{2} \omega_{0}}{2}\right) t_{1}} e^{i\left(\varepsilon_{2}+\frac{n_{2} \omega_{0}}{2}\right) t_{2}} \\
& \times e^{-i\left(\varepsilon_{3}+\frac{m_{3} \omega_{0}}{2}\right) t_{2}} e^{i\left(\varepsilon_{3}+\frac{n_{3} \omega_{0}}{2}\right) t^{\prime}} g_{m_{1} n_{1}}^{\tau \tau, r}\left(\varepsilon_{1}\right) \Sigma_{m_{2} n_{2}}^{\tau, r}\left(\varepsilon_{2}\right) G_{m_{3} n_{3}}^{\tau \tau^{\prime}, r}\left(\varepsilon_{3}\right) \\
& =\frac{1}{2 \pi} \sum_{n_{1} n_{2} n_{3}} \int d \varepsilon_{1} d \varepsilon_{2} d \varepsilon_{3} e^{-i\left(\varepsilon_{1}+\frac{m_{1} \omega_{0}}{2}\right) t} \delta\left(\varepsilon_{1}+\frac{n_{1} \omega_{0}}{2}-\varepsilon_{2}-\frac{m_{2} \omega_{0}}{2}\right) \delta\left(\varepsilon_{2}+\frac{n_{2} \omega_{0}}{2}-\varepsilon_{3}-\frac{m_{3} \omega_{0}}{2}\right) \\
& \times e^{i\left(\varepsilon_{3}+\frac{n_{3} \omega_{0}}{2}\right) t^{\prime}} g_{m_{1} n_{1}}^{\tau \tau, r}\left(\varepsilon_{1}\right) \Sigma_{m_{2} n_{2}}^{\tau, r}\left(\varepsilon_{2}\right) G_{m_{3} n_{3}}^{\tau \tau^{\prime}, r}\left(\varepsilon_{3}\right) \\
& =\frac{1}{2 \pi} \sum_{n_{1} n_{2} n_{3}} \int d \varepsilon_{2} d \varepsilon_{3} e^{-i\left(\varepsilon_{2}+\frac{m_{2} \omega_{0}}{2}-\frac{n_{1} \omega_{0}}{2}+\frac{m_{1} \omega_{0}}{2}\right) t} \delta\left(\varepsilon_{2}+\frac{n_{2} \omega_{0}}{2}-\varepsilon_{3}-\frac{m_{3} \omega_{0}}{2}\right) \\
& \times e^{i\left(\varepsilon_{3}+\frac{n_{3} \omega_{0}}{2}\right) t^{\prime}} g_{m_{1} n_{1}}^{\tau \tau, r}\left(\varepsilon_{2}+\frac{m_{2} \omega_{0}}{2}-\frac{n_{1} \omega_{0}}{2}\right) \Sigma_{m_{2} n_{2}}^{\tau, r}\left(\varepsilon_{2}\right) G_{m_{3} n_{3}}^{\tau \tau^{\prime}, r}\left(\varepsilon_{3}\right) \\
& =\frac{1}{2 \pi} \sum_{n_{1} n_{2} n_{3}} \int d \varepsilon_{2} e^{-i\left(\varepsilon_{2}+\frac{m_{2} \omega_{0}}{2}-\frac{n_{1} \omega_{0}}{2}+\frac{m_{1} \omega_{0}}{2}\right) t} e^{i\left(\varepsilon_{2}+\frac{n_{2} \omega_{0}}{2}-\frac{m_{3} \omega_{0}}{2}+\frac{n_{3} \omega_{0}}{2}\right) t^{\prime}} \\
& \times g_{m_{1} n_{1}}^{\tau \tau, r}\left(\varepsilon_{2}+\frac{m_{2} \omega_{0}}{2}-\frac{n_{1} \omega_{0}}{2}\right) \Sigma_{m_{2} n_{2}}^{\tau, r}\left(\varepsilon_{2}\right) G_{m_{3} n_{3}}^{\tau \tau^{\prime}, r}\left(\varepsilon_{2}+\frac{n_{2} \omega_{0}}{2}-\frac{m_{3} \omega_{0}}{2}\right) \\
& =\frac{1}{2 \pi} \sum_{n_{1} n_{2} n_{3}} \int d \varepsilon_{2} e^{-i\left[\varepsilon_{2}+\left(m_{2}-n_{1}+m_{1}\right) \frac{\omega_{0}}{2}\right] t} e^{i\left[\varepsilon_{2}+\left(n_{2}-m_{3}+n_{3}\right) \frac{\omega_{0}}{2}\right] t^{\prime}} \\
& \times g_{m_{1} n_{1}}^{\tau \tau, r}\left[\varepsilon_{2}+\left(m_{2}-n_{1}\right) \frac{\omega_{0}}{2}\right] \Sigma_{m_{2} n_{2}}^{\tau, r}\left(\varepsilon_{2}\right) G_{m_{3} n_{3}}^{\tau \tau^{\prime}, r}\left[\varepsilon_{2}+\left(n_{2}-m_{3}\right) \frac{\omega_{0}}{2}\right] .
\end{aligned}
$$

Replacing $\varepsilon_{2}-\frac{n_{1} \omega_{0}}{2}$ by $\varepsilon_{2}$ we arrive at

$$
\begin{aligned}
& \int d t_{1} \int d t_{2} g^{\tau \tau, r}\left(t-t_{1}\right) \Sigma^{\tau, r}\left(t_{1}, t_{2}\right) G^{\tau \tau^{\prime}, r}\left(t_{2}, t^{\prime}\right) \\
= & \frac{1}{2 \pi} \sum_{n_{1} n_{2} n_{3}} \int d \varepsilon_{2} e^{-i\left[\varepsilon_{2}+\left(m_{2}+m_{1}\right) \frac{\omega_{0}}{2}\right] t} e^{i\left[\varepsilon_{2}+\left(n_{1}+n_{2}-m_{3}+n_{3}\right) \frac{\omega_{0}}{2}\right] t^{\prime}} \\
& \times g_{m_{1} n_{1}}^{\tau \tau, r}\left(\varepsilon_{2}+m_{2} \frac{\omega_{0}}{2}\right) \Sigma_{m_{2} n_{2}}^{\tau, r}\left(\varepsilon_{2}+n_{1} \frac{\omega_{0}}{2}\right) G_{m_{3} n_{3}}^{\tau \tau^{\prime}, r}\left[\varepsilon_{2}+\left(n_{1}+n_{2}-m_{3}\right) \frac{\omega_{0}}{2}\right] .
\end{aligned}
$$


Let $m_{2}=k_{1}-m_{1}$ and $n_{3}=k_{2}-n_{1}-n_{2}+m_{3}$, then

$$
\begin{aligned}
& \int d t_{1} \int d t_{2} g^{\tau \tau, r}\left(t-t_{1}\right) \Sigma^{\tau, r}\left(t_{1}, t_{2}\right) G^{\tau \tau^{\prime}, r}\left(t_{2}, t^{\prime}\right) \\
= & \frac{1}{2 \pi} \sum_{n_{1} n_{2} k_{2}} \int d \varepsilon_{2} e^{-i\left(\varepsilon_{2}+k_{1} \frac{\omega_{0}}{2}\right) t} e^{i\left(\varepsilon_{2}+k_{2} \frac{\omega_{0}}{2}\right) t^{\prime}} \\
& \times g_{m_{1}}^{\tau \tau, r}\left[\varepsilon_{2}+\left(k_{1}-m_{1}\right) \frac{\omega_{0}}{2}\right] \Sigma_{k_{1}-m_{1}, n_{2}}^{\tau, r}\left(\varepsilon_{2}+n_{1} \frac{\omega_{0}}{2}\right) \\
& G_{m_{3}, k_{2}-k_{2}-n_{1}-n_{2}+m_{3}}^{\tau \tau^{\prime}}\left[\varepsilon_{2}+\left(n_{1}+n_{2}-m_{3}\right) \frac{\omega_{0}}{2}\right] \\
= & \frac{1}{2 \pi} \sum_{n_{1} n_{2} k_{2}}^{\int} \int d \varepsilon_{2} e^{-i\left(\varepsilon_{2}+k_{1} \frac{\omega_{0}}{2}\right) t} e^{i\left(\varepsilon_{2}+k_{2} \frac{\omega_{0}}{2}\right) t^{\prime}} \\
& \times g_{k_{1}, n_{1}+k_{1}-m_{1}}^{\tau \tau, r}\left(\varepsilon_{2}\right) \Sigma_{n_{1}+k_{1}-m_{1}, n_{1}+n_{2}}^{\tau, r}\left(\varepsilon_{2}\right) G_{n_{1}+n_{2}, k_{2}}^{\tau \tau^{\prime}, r}\left(\varepsilon_{2}\right) \\
= & \frac{1}{2 \pi} \sum_{n_{1} n_{2} k_{2}} \int d \varepsilon_{2} e^{-i\left(\varepsilon_{2}+k_{1} \frac{\omega_{0}}{2}\right) t} e^{i\left(\varepsilon_{2}+k_{2} \frac{\omega_{0}}{2}\right) t^{\prime}} g_{k_{1}, n_{1}}^{\tau \tau, r}\left(\varepsilon_{2}\right) \sum_{n_{1}, n_{2}}^{\tau, r}\left(\varepsilon_{2}\right) G_{n_{2}, k_{2}}^{\tau \tau^{\prime}, r}\left(\varepsilon_{2}\right) .
\end{aligned}
$$

Substituting Eq.(D.36) in Eq.(D.10) we find the expression

$$
\mathcal{G}_{m n}^{\tau \tau^{\prime}, r}(\varepsilon)=\delta_{\tau \tau^{\prime}} g_{m n}^{\tau \tau, r}(\varepsilon)+\sum_{n_{1} n_{2}} g_{m, n_{1}}^{\tau \tau, r}(\varepsilon) \sum_{n_{1}, n_{2}}^{\tau, r}(\varepsilon) \mathcal{G}_{n_{2}, n}^{\tau \tau^{\prime}, r}(\varepsilon)
$$

The corresponding matrix equations read

$$
\begin{aligned}
& \left(\begin{array}{ll}
\mathcal{G}_{m \pi^{\prime}, r}^{\tau \tau^{\prime},+}(\varepsilon) & \mathcal{G}_{m n ;,+-}^{\tau \tau^{\prime}, r}(\varepsilon) \\
\mathcal{G}_{m n ;-++}^{\tau \tau^{\prime}, r}(\varepsilon) & \mathcal{G}_{m n ;--}^{\tau \tau^{\prime}, r}(\varepsilon)
\end{array}\right)=\delta_{\tau \tau^{\prime}} \delta_{m n}\left(\begin{array}{cc}
g_{m n ;++}^{\tau \tau, r}(\varepsilon) & g_{m n ;+-}^{\tau \tau, r}(\varepsilon) \\
g_{m n ;-+}^{\tau \tau, r}(\varepsilon) & g_{m n ;--}^{\tau \tau, r}(\varepsilon)
\end{array}\right) \\
& +\sum_{n_{1} n_{2}} \delta_{m n_{1}}\left(\begin{array}{cc}
g_{m n_{1} ;++}^{\tau \tau, r}(\varepsilon) & g_{m n_{1} ;+-}^{\tau \tau, r}(\varepsilon) \\
g_{m n_{1} ;-+}^{\tau \tau, r}(\varepsilon) & g_{m n_{1} ;--}^{\tau \tau, r}(\varepsilon)
\end{array}\right) \Sigma^{\tau, r}\left(\begin{array}{cc}
\delta_{n_{1} n_{2}} & \delta_{n_{2}, n_{1}+2} \\
\delta_{n_{2}, n_{1}-2} & \delta_{n_{1} n_{2}}
\end{array}\right)
\end{aligned}
$$

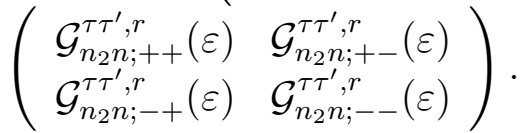

This means that

$$
\begin{aligned}
& \left(\begin{array}{ll}
\mathcal{G}_{m n ;+++}^{\tau \tau^{\prime}, r}(\varepsilon) & \mathcal{G}_{m n ;+,-}^{\tau \tau^{\prime}, r}(\varepsilon) \\
\mathcal{G}_{m n ;-+}^{\tau \tau^{\prime}, r}(\varepsilon) & \mathcal{G}_{m n ;--}^{\tau \tau^{\prime}, r}(\varepsilon)
\end{array}\right)=\delta_{\tau \tau^{\prime}} \delta_{m n}\left(\begin{array}{cc}
g_{m n ;,++}^{\tau \tau, r}(\varepsilon) & g_{m n ;+,}^{\tau \tau, r}(\varepsilon) \\
g_{m n ;-++}^{\tau \tau, r}(\varepsilon) & g_{m n ;--}^{\tau \tau, r}(\varepsilon)
\end{array}\right) \\
& +\sum_{n_{2}}\left(\begin{array}{cc}
g_{m m ;++}^{\tau \tau, r}(\varepsilon) & g_{m m ;+-}^{\tau \tau, r}(\varepsilon) \\
g_{m m ;-+}^{\tau \tau, r}(\varepsilon) & g_{m m ;--}^{\tau \tau, r}(\varepsilon)
\end{array}\right) \Sigma^{\tau, r}\left(\begin{array}{cc}
\delta_{m n_{2}} & \delta_{n_{2}, m+2} \\
\delta_{n_{2}, m-2} & \delta_{m n_{2}}
\end{array}\right) \\
& \left(\begin{array}{ll}
\mathcal{G}_{n_{2} n ;+++}^{\tau \tau^{\prime}, r}(\varepsilon) & \mathcal{G}_{n_{2} n ;+,-}^{\tau \tau^{\prime}, r}(\varepsilon) \\
\mathcal{G}_{n_{2} n ;-+}^{\tau \tau^{\prime}, r}(\varepsilon) & \mathcal{G}_{n_{2} n ;--}^{\tau \tau^{\prime}, r}(\varepsilon)
\end{array}\right) \\
& =\delta_{\tau \tau^{\prime}} \delta_{m n}\left(\begin{array}{cc}
g_{m n ;++}^{\tau \tau, r}(\varepsilon) & g_{m n ;+-}^{\tau \tau, r}(\varepsilon) \\
g_{m n ;-+}^{\tau \tau, r}(\varepsilon) & g_{m n ;--}^{\tau \tau, r}(\varepsilon)
\end{array}\right) \\
& +\left(\begin{array}{cc}
g_{m m ;+++}^{\tau \tau, r}(\varepsilon) \Sigma^{\tau, r} & g_{m m ;+-}^{\tau \tau, r}(\varepsilon) \Sigma^{\tau, r} \\
g_{m m ;-++}^{\tau \tau, r}(\varepsilon) \Sigma^{\tau, r} & g_{m m ;--}^{\tau \tau, r}(\varepsilon) \Sigma^{\tau, r}
\end{array}\right)\left(\begin{array}{ll}
\mathcal{G}_{m n ;+++}^{\tau \tau^{\prime}, r}(\varepsilon) & \mathcal{G}_{m n ;+-}^{\tau \tau^{\prime}, r}(\varepsilon) \\
\mathcal{G}_{m n ;-+}^{\tau \tau^{\prime}, r}(\varepsilon) & \mathcal{G}_{m n ;--}^{\tau \tau^{\prime}, r}(\varepsilon)
\end{array}\right) \\
& +\left(\begin{array}{ll}
g_{m m ;+-}^{\tau \tau, r}(\varepsilon) \Sigma^{\tau, r} & 0 \\
g_{m m ;--}^{\tau \tau, r}(\varepsilon) \Sigma^{\tau, r} & 0
\end{array}\right)\left(\begin{array}{ll}
\mathcal{G}_{m-2, n ;++}^{\tau \tau^{\prime}, r}(\varepsilon) & \mathcal{G}_{m-2, n ;+-}^{\tau \tau^{\prime}, r}(\varepsilon) \\
\mathcal{G}_{m-2, n ;-+}^{\tau \tau^{\prime}, r}(\varepsilon) & \mathcal{G}_{m-2, n ;--}^{\tau \tau^{\prime}, r}(\varepsilon)
\end{array}\right) \\
& +\left(\begin{array}{cc}
0 & g_{m m ;++}^{\tau \tau, r}(\varepsilon) \Sigma^{\tau, r} \\
0 & g_{m m ;-+}^{\tau \tau, r}(\varepsilon) \Sigma^{\tau, r}
\end{array}\right)\left(\begin{array}{ll}
\mathcal{G}_{m+2, n ;++}^{\tau \tau^{\prime}, r}(\varepsilon) & \mathcal{G}_{m+2, n ;+-}^{\tau \tau^{\prime}, r}(\varepsilon) \\
\mathcal{G}_{m+2, n ;-+}^{\tau \tau^{\prime}, r}(\varepsilon) & \mathcal{G}_{m+2, n ;--}^{\tau \tau^{\prime}, r}(\varepsilon)
\end{array}\right) .
\end{aligned}
$$

Eq.(D.39) is simply written as

$$
\mathcal{G}_{m, n}=g_{m n}+\widetilde{\epsilon}_{m} \mathcal{G}_{m, n}+V_{m, m-2} \mathcal{G}_{m-2, n}+V_{m, m+2} \mathcal{G}_{m+2, n}
$$


Laser-assisted spin-polarized transport in graphene tunnel junctions

with $g$ being diagonal. Furthermore, we deduce that

$$
\mathcal{G}_{N, n}=\widetilde{\epsilon}_{N} \mathcal{G}_{N, n}+V_{N, N-2} \mathcal{G}_{N-2, n}, \quad N>n
$$

which means that

$$
\mathcal{G}_{N, n}=B_{N} \mathcal{G}_{N-2, n}, \quad B_{N}=\left(I-\widetilde{\epsilon}_{N}\right)^{-1} V_{N, N-2} . \quad N>n
$$

Furthermore, it is straightforward to see that

$$
\begin{gathered}
\mathcal{G}_{N-2, n}=B_{N-2} G_{N-4, n}^{r}, \quad B_{N-2}=\left[I-\widetilde{\epsilon}_{N-2}-V_{N-2, N} B_{N}\right]^{-1} V_{N-2, N-4}, \quad N-1>n(\mathrm{D} .43) \\
\mathcal{G}_{m, n}=B_{m} \mathcal{G}_{m-2, n}, \quad B_{m}=\left(I-\widetilde{\epsilon}_{m}-V_{m, m+2} B_{m+2}\right)^{-1} V_{m, m-2} . \quad m>n(\mathrm{D} .44)
\end{gathered}
$$

Likewise we deduce that

$$
\begin{gathered}
\mathcal{G}_{-N, n}=F_{-N} \mathcal{G}_{-N+2, n}, \quad F_{-N}=\left(I-\widetilde{\epsilon}_{-N}\right)^{-1} V_{-N,-N+2}, \\
\mathcal{G}_{-N+2, n}=F_{-N+2} \mathcal{G}_{-N+4, n}, \quad F_{-N+2} \\
=\left(I-\widetilde{\epsilon}_{-N+2}-V_{-N+2,-N} F_{-N}\right)^{-1} V_{-N+2,-N+4},
\end{gathered}
$$

and

$$
\mathcal{G}_{m, n}=F_{m} \mathcal{G}_{m+2, n}, \quad F_{m}=\left(I-\widetilde{\epsilon}_{m}-V_{m, m-2} F_{m-2}\right)^{-1} V_{m, m+2} . \quad m<n
$$

Using $\mathcal{G}_{m, n}(\varepsilon)=\mathcal{G}_{m-n, 0}\left(\varepsilon+n \omega_{0} / 2\right)$ from $\mathcal{G}_{m, 0}(\varepsilon)$ all the elements of the matrix $\mathcal{G}_{m n}(\varepsilon)$ follow. Denoting $\mathcal{G}_{m 0}(\varepsilon) \equiv \mathcal{G}_{m}$, i.e. $\mathcal{G}_{0}=g_{00}+\widetilde{\epsilon}_{0} \mathcal{G}_{0}+V_{0,-2} \mathcal{G}_{-2}+V_{0,2} \mathcal{G}_{2}$ and combining Eqs.(D.44) and (D.47) we find

$$
\begin{aligned}
\mathcal{G}_{0}= & g_{00}+\widetilde{\epsilon}_{0} \mathcal{G}_{0}+V_{0,-2} \mathcal{G}_{-2}+V_{0,2} \mathcal{G}_{2} \\
= & g_{00}+\widetilde{\epsilon}_{0} \mathcal{G}_{0}+V_{0,-2} F_{-2} \mathcal{G}_{0}+V_{0,2} B_{2} \mathcal{G}_{0} \\
= & {\left[I-\widetilde{\epsilon}_{0}-V_{0,-2} F_{-2}-V_{0,2} B_{2}\right]^{-1} g_{00} . }
\end{aligned}
$$

With this we conclude that

$$
\begin{gathered}
\mathcal{G}_{-2 m}=\left(\prod_{i=1}^{m} F_{-2 i}\right) \mathcal{G}_{0}, \quad m \geq 1, \\
\mathcal{G}_{2 m}=\left(\prod_{i=1}^{m} B_{2 i}\right) \mathcal{G}_{0}, \quad m \geq 1, \\
\mathcal{G}_{m, n}(\varepsilon)=\mathcal{G}_{m-n, 0}\left(\varepsilon+n \omega_{0} / 2\right),
\end{gathered}
$$

where

$$
\begin{aligned}
& B_{m}=\left(I-\widetilde{\epsilon}_{m}-V_{m, m+2} B_{m+2}\right)^{-1} V_{m, m-2}, \\
& F_{m}=\left(I-\widetilde{\epsilon}_{m}-V_{m, m-2} F_{m-2}\right)^{-1} V_{m, m+2} .
\end{aligned}
$$




\section{Appendix E. The current expressed in terms of Fourier-transformed Green's functions}

Let $\Gamma_{L}^{\tau}=\gamma \Gamma_{R}^{\tau}$, and $N(t)$ be the occupation of the central region. Using the continuity equation and that the spin channels are decoupled we find for the current

$$
\begin{aligned}
& I_{L}^{\tau}(t)=\frac{1}{1+\gamma} I_{L}^{\tau}(t)+\frac{\gamma}{1+\gamma}\left[e^{\left.\frac{d N_{\tau}(t)}{d t}-I_{R}^{\tau}(t)\right]}\right. \\
= & \frac{\gamma}{1+\gamma} e \frac{d N_{\tau}(t)}{d t}-\frac{\gamma}{1+\gamma} \frac{e}{\hbar} \Re \sum_{s s^{\prime}} \int \frac{d \varepsilon}{2 \pi} \int d t_{1} e^{-i \varepsilon\left(t_{1}-t\right)} e^{-\frac{i s \omega_{0} t}{2}} e^{\frac{i s^{\prime} \omega_{0} t_{1}}{2}} \\
\times & \left.\times 2 \mathcal{G}_{s, s^{\prime}}^{\tau \tau, r}\left(t, t_{1}\right) i \Gamma_{R}^{\tau}\left[f_{L}(\varepsilon)-f_{R}(\varepsilon)\right]\right\} \\
= & \frac{\gamma}{1+\gamma} e \frac{d N_{\tau}(t)}{d t}-\frac{\gamma}{1+\gamma} \frac{e}{\hbar} \Re \sum_{s s^{\prime}} \int \frac{d \varepsilon}{2 \pi} \int d t_{1} e^{-i \varepsilon\left(t_{1}-t\right)} e^{-\frac{i s \omega_{0} t}{2}} e^{\frac{i s^{\prime} \omega_{0} t_{1}}{2}} \sum_{n} \int \frac{d \varepsilon^{\prime}}{2 \pi} e^{-i \varepsilon^{\prime}\left(t-t_{1}\right)} e^{i n \omega_{0}\left(t+t_{1}\right) / 2} \\
& \times\left\{2 \mathcal{G}_{s, s^{\prime}}^{\tau \tau, r}\left(\varepsilon^{\prime}-n \omega_{0} / 2, \varepsilon^{\prime}+n \omega_{0} / 2\right) i \Gamma_{R}^{\tau}\left[f_{L}(\varepsilon)-f_{R}(\varepsilon)\right]\right\} \\
= & \frac{\gamma}{1+\gamma} e \frac{d N_{\tau}(t)}{d t}-\frac{\gamma}{1+\gamma} \frac{e}{\hbar} \Re \sum_{n} \sum_{s s^{\prime}} \int \frac{d \varepsilon}{2 \pi} \int \frac{d \varepsilon^{\prime}}{2 \pi} \int d t_{1} e^{-\frac{i s \omega_{0} t}{2}} e^{\frac{i s^{\prime} \omega_{0} t_{1}}{2}} e^{-i\left(\varepsilon^{\prime}-\varepsilon\right)\left(t-t_{1}\right)} e^{i n \omega_{0}\left(t+t_{1}\right) / 2} \\
= & \frac{\gamma}{1+\gamma} e \frac{\left.d \mathcal{G}_{s, s_{\tau}^{\prime}}^{\tau \tau}\left(\varepsilon^{\prime}-n \omega_{0} / 2, \varepsilon^{\prime}+n \omega_{0} / 2\right) i \Gamma_{R}^{\tau}\left[f_{L}(\varepsilon)-f_{R}(\varepsilon)\right]\right\}}{d t}-\frac{\gamma}{1+\gamma} \frac{e}{\hbar} \Re \sum_{s s^{\prime}} \sum_{n} \int \frac{d \varepsilon^{\prime}}{2 \pi} \int \frac{d \varepsilon}{2 \pi} \int_{-\infty}^{t} d t_{1} e^{i\left(\varepsilon-\varepsilon^{\prime}+(n-s) \omega_{0} / 2\right) t} e^{i\left(-\varepsilon+\varepsilon^{\prime}+\left(n+s^{\prime}\right) \omega_{0} / 2\right) t_{1}} \\
& \times\left\{2 \mathcal{G}_{s, s^{\prime}}^{\tau \tau, r}\left(\varepsilon^{\prime}-n \omega_{0} / 2, \varepsilon^{\prime}+n \omega_{0} / 2\right) i \Gamma_{R}^{\tau}\left[f_{L}(\varepsilon)-f_{R}(\varepsilon)\right]\right\} .
\end{aligned}
$$

Defining $\varepsilon^{\prime \prime}=\varepsilon^{\prime}+\frac{\left(s^{\prime}+n\right) \omega_{0}}{2}$, we may rewrite the integral over $t_{1}$ as

$$
\begin{aligned}
& \int_{-\infty}^{t} d t_{1} e^{i t_{1}\left(-\varepsilon+\varepsilon^{\prime}+\left(n+s^{\prime}\right) \omega_{0} / 2\right)} \\
= & \int_{-\infty}^{t} d t_{1} \exp \left[i t_{1}\left(\varepsilon^{\prime \prime}-\varepsilon\right)\right] \\
= & \int_{-\infty}^{\infty} d t_{1} \theta\left(t-t_{1}\right) \exp \left[i t_{1}\left(\varepsilon^{\prime \prime}-\varepsilon\right)\right] \\
= & \int_{-\infty}^{\infty} d t_{1} \int \frac{d \omega^{\prime}}{2 \pi i} \frac{\exp \left[i \omega^{\prime}\left(t-t_{1}\right)\right]}{\omega^{\prime}-i \eta} \exp \left[i t_{1}\left(\varepsilon^{\prime \prime}-\varepsilon\right)\right] \\
= & \int \frac{d \omega^{\prime}}{2 \pi i} \frac{\exp \left(i \omega^{\prime} t\right)}{\omega^{\prime}-i \eta} \underbrace{\int_{-\infty}^{\int^{\prime}} d t_{1} \exp \left[i t_{1}\left(\varepsilon^{\prime \prime}-\varepsilon-\omega^{\prime}\right)\right]}_{2 \pi \delta\left(\varepsilon^{\prime \prime}-\varepsilon-\omega^{\prime}\right)} \\
= & -i \frac{\exp \left[i\left(\varepsilon^{\prime \prime}-\varepsilon\right) t\right]}{\varepsilon^{\prime \prime}-\varepsilon-i \eta}=-i \frac{\exp \left\{i\left[\varepsilon^{\prime}-\varepsilon+\left(s^{\prime}+n\right) \omega_{0} / 2\right] t\right\}}{\varepsilon^{\prime}-\varepsilon+\left(s^{\prime}+n\right) \omega_{0} / 2-i \eta} .
\end{aligned}
$$

Upon substituting this expression into Eq. (E.1) we find

$$
\begin{aligned}
I_{L}^{\tau}(t) & =\frac{\gamma}{1+\gamma} e \frac{d N_{\tau}(t)}{d t}-\frac{\gamma}{1+\gamma} \frac{e}{\hbar} \Re \sum_{n s s^{\prime}} \exp \left(i t \omega_{0}\left[n+\left(s^{\prime}-s\right) / 2\right]\right) \\
& \times \int \frac{d \varepsilon}{2 \pi} 2 i \Gamma_{R}^{\tau}\left[f_{L}(\varepsilon)-f_{R}(\varepsilon)\right] \underbrace{\int \frac{d \omega}{2 \pi i} \frac{\mathcal{G}_{s s^{\prime}}^{\tau \tau, r}\left(\omega-n \omega_{0} / 2, \omega+n \omega_{0} / 2\right)}{\omega-\varepsilon+\left(n+s^{\prime}\right) \omega_{0} / 2-i \eta}}_{\text {Integral(II) }} .
\end{aligned}
$$

Integral (II) can be evaluated by means of residue method to give

$$
\begin{aligned}
I_{L}^{\tau}(t) & =\frac{\gamma}{1+\gamma} e \frac{d N_{\tau}(t)}{d t}-\frac{\gamma}{1+\gamma} \frac{e}{\hbar} \Re \sum_{n s s^{\prime}} \int \frac{d \varepsilon}{2 \pi} 2 i e^{i\left(2 n-s+s^{\prime}\right) \omega_{0} t / 2} \\
& \times\left\{\mathcal{G}_{s s^{\prime}}^{\tau \tau, r}\left[\varepsilon-\left(2 n+s^{\prime}\right) \omega_{0} / 2, \varepsilon-s^{\prime} \omega_{0} / 2\right] \Gamma_{R}^{\tau}\left[f_{L}(\varepsilon)-f_{R}(\varepsilon)\right]\right\} .
\end{aligned}
$$


The time-averaged current is thus given by

$$
\begin{gathered}
\left\langle I_{L}^{\tau}\right\rangle=\frac{\gamma}{1+\gamma} e\left\langle\frac{d N_{\tau}(t)}{d t}\right\rangle-\frac{\gamma}{1+\gamma} \frac{e}{\hbar} \Re \sum_{s s^{\prime}} \sum_{n} \int \frac{d \varepsilon}{2 \pi} \delta_{2 n, s-s^{\prime}} \\
\times\left\{2 \mathcal{G}_{s, s^{\prime}}^{\tau \tau, r}\left[\varepsilon-\left(2 n+s^{\prime}\right) \omega_{0} / 2, \varepsilon-s^{\prime} \omega_{0} / 2\right] i \Gamma_{R}^{\tau}\left[f_{L}(\varepsilon)-f_{R}(\varepsilon)\right]\right\} \\
=\frac{\gamma}{1+\gamma}\left\{e\left\langle\frac{d N_{\tau}(t)}{d t}\right\rangle-\frac{e}{\hbar} \Re \sum_{s s^{\prime}} \int \frac{d \varepsilon}{2 \pi} 2 \mathcal{G}_{s, s^{\prime}}^{\tau \tau, r}\left(\varepsilon-s \omega_{0} / 2, \varepsilon-s^{\prime} \omega_{0} / 2\right) i \Gamma_{R}^{\tau}\left[f_{L}(\varepsilon)-f_{R}(\varepsilon)\right]\right\} \\
=-\frac{\gamma}{1+\gamma} \frac{i e}{\hbar} \sum_{s s^{\prime}} \int \frac{d \varepsilon}{2 \pi} \Gamma_{R}^{\tau}\left[f_{L}(\varepsilon)-f_{R}(\varepsilon)\right] \\
\left.\quad \times\left[\mathcal{G}_{s, s^{\prime}}^{\tau \tau, r}-s \omega_{0} / 2, \varepsilon-s^{\prime} \omega_{0} / 2\right)-\mathcal{G}_{s, s^{\prime}}^{\tau \tau, a}\left(\varepsilon-s \omega_{0} / 2, \varepsilon-s^{\prime} \omega_{0} / 2\right)\right] \\
=-\frac{\gamma}{1+\gamma} \frac{i e}{\hbar} \sum_{s s^{\prime}} \int \frac{d \varepsilon}{2 \pi}\left[\mathcal{G}_{-s,-s^{\prime} ; s s^{\prime}}^{\tau \tau}(\varepsilon)-\mathcal{G}_{-s,-s^{\prime} ; s s^{\prime}}(\varepsilon)\right] \Gamma_{R}^{\tau}\left[f_{L}(\varepsilon)-f_{R}(\varepsilon)\right] \\
=-\frac{i e}{\hbar} \sum_{s s^{\prime}} \int \frac{d \varepsilon}{2 \pi}\left[\mathcal{G}_{-s,-s^{\prime} ; s s^{\prime}}^{\tau \tau, r}(\varepsilon)-\mathcal{G}_{-s,-s^{\prime} ; s s^{\prime}}^{\tau \tau, a}(\varepsilon)\right] \frac{\Gamma_{L}^{\tau} \Gamma_{R}^{\tau}}{\Gamma_{L}^{\tau}+\Gamma_{R}^{\tau}}\left[f_{L}(\varepsilon)-f_{R}(\varepsilon)\right] .
\end{gathered}
$$

With this we conclude that

$$
\begin{aligned}
\left\langle I_{L}\right\rangle & =\left\langle I_{L}^{\uparrow}\right\rangle+\left\langle I_{L}^{\downarrow}\right\rangle \\
& =-\frac{i e}{\hbar} \sum_{s s^{\prime} \tau} \int \frac{d \varepsilon}{2 \pi}\left[\mathcal{G}_{-s,-s^{\prime} ; s s^{\prime}}^{\tau \tau, r}(\varepsilon)-\mathcal{G}_{-s,-s^{\prime} ; s s^{\prime}}^{\tau \tau, a}(\varepsilon)\right] \frac{\Gamma_{L}^{\tau} \Gamma_{R}^{\tau}}{\Gamma_{L}^{\tau}+\Gamma_{R}^{\tau}}\left[f_{L}(\varepsilon)-f_{R}(\varepsilon)\right],
\end{aligned}
$$

For numerical implementation we use the expression (we employ $\Gamma_{L}=\Gamma_{R}$, i.e. $\gamma=1)$

$$
\begin{aligned}
& \left\langle I_{L}\right\rangle=-\frac{i e}{2 h} \sum_{\tau} \int d \varepsilon\left\{\left[\mathcal{G}_{-1,-1 ;++}^{\tau \tau, r}(\varepsilon)-\mathcal{G}_{-1,-1 ;++}^{\tau \tau, a}(\varepsilon)\right]+\left[\mathcal{G}_{-1,1 ;+-}^{\tau \tau, r}(\varepsilon)-\mathcal{G}_{-1,1 ;+-}^{\tau \tau, a}(\varepsilon)\right]\right. \\
+ & {\left.\left[\mathcal{G}_{1,-1 ;-++}^{\tau \tau, r}(\varepsilon)-\mathcal{G}_{1,-1 ;-+}^{\tau \tau, a}(\varepsilon)\right]+\left[\mathcal{G}_{1,1 ;--}^{\tau \tau, r}(\varepsilon)-\mathcal{G}_{1,1 ;--}^{\tau \tau, a}(\varepsilon)\right]\right\} \Gamma_{R}^{\tau}\left[f_{L}(\varepsilon)-f_{R}(\varepsilon)\right] } \\
= & -\frac{i e}{2 h} \sum_{\tau} \int d \varepsilon\left\{\left[\mathcal{G}_{00 ;++}^{\tau \tau, r}\left(\varepsilon-\frac{\omega_{0}}{2}\right)-\mathcal{G}_{00 ;+,+}^{\tau \tau, a}\left(\varepsilon-\frac{\omega_{0}}{2}\right)\right]+\left[\mathcal{G}_{-20 ;+-}^{\tau \tau, r}\left(\varepsilon+\frac{\omega_{0}}{2}\right)-\mathcal{G}_{-20 ;+-}^{\tau \tau, a}\left(\varepsilon+\frac{\omega_{0}}{2}\right)\right]\right. \\
+ & {\left.\left[\mathcal{G}_{20 ;,-+}^{\tau \tau, r}\left(\varepsilon-\frac{\omega_{0}}{2}\right)-\mathcal{G}_{20 ;-+}^{\tau \tau, a}\left(\varepsilon-\frac{\omega_{0}}{2}\right)\right]+\left[\mathcal{G}_{00 ;--}^{\tau \tau, r}\left(\varepsilon+\frac{\omega_{0}}{2}\right)-\mathcal{G}_{00 ;--}^{\tau \tau, a}\left(\varepsilon+\frac{\omega_{0}}{2}\right)\right]\right\} } \\
\times & \Gamma_{R}^{\tau}\left[f_{L}(\varepsilon)-f_{R}(\varepsilon)\right] .
\end{aligned}
$$

